\title{
Generalized Seiberg-Witten equations and hyperKähler geometry
}

\author{
Dissertation \\ zur Erlangung des Doktorgrades \\ der Mathematisch-Naturwissenschaftlichen Fakultäten \\ der Georg-August-Universität zu Göttingen
}

vorgelegt von

Andriy Haydys

aus

Lviv, Ukraine

Göttingen 2006 
D7

Referent: Prof. Dr. Victor Pidstrygach

Korreferent: Prof. Dr. Thomas Schick

Tag der mündlichen Prüfung: 09.02.2006 


\begin{abstract}
In this thesis we study a certain generalization of the gauge-theoretical Seiberg-Witten equations over a source 4-manifold $X$. The generalization involves a hyperKähler manifold $M$ with certain symmetries and a nonlinear Dirac operator $\mathcal{D}$ acting on equivariant maps $u$ (called spinors) with values in $M$.

We prove a classification theorem for such hyperKähler manifolds and propose a new method for their construction. This allows us to obtain new examples of hyperKähler and closely related to them quaternionic Kähler manifolds. Our construction is quite explicit and, in some cases, this allows to obtain not only existence results but also hyper- and quaternionic-Kähler structures themselves.

We also prove that harmonic spinors, i.e. solutions of the equation $\mathcal{D} u=$ 0 , are closely related to solutions of the so-called Cauchy-Riemann-Fueter equation. We then prove that solutions of the Cauchy-Riemann-Fueter equation, which are believed to be a "right" analogue of holomorphic maps in quaternionic context, are exactly those maps, whose differential has no triholomorphic component. Hence we introduce the term "aholomorphic" for such maps. It is also shown that harmonic spinors can be regarded as twisted version (in an appropriate sense) of aholomorphic maps.

The last part of the thesis is devoted to the generalized Seiberg-Witten equations over Kähler surfaces. In this case we prove that the space of solutions has a holomorphic description (in the usual complex sense). Further, if $X$ is a product of two holomorphic curves we show (modulo adiabatic limit conjecture) that there exists a relation between holomorphic curves (in the sense of Gromov theory), the symplectic vortex equations [18] and the generalized Seiberg-Witten equations.
\end{abstract}




\section{Acknowledgement}

I would like to thank all those, who helped and supported me during the long period of work on this thesis. First and foremost, I want to thank my supervisor Prof. Victor Pidstrygach, who interested me in the beautiful world of geometry. I am especially gratefull to Prof. Pidstrygach for providing freedom in conducting my research and being always available when I needed his help or advice.

I also wish to acknowledge Graduiertenkolleg "Groups and Geometry" of DFG for financial support and stimulating atmosphere. Many thanks due to my friends and colleagues with whom I spent a lot of funny and interesting hours. Special thanks due to Juan Cervinõ, Annika Eickhoff-Schachtebeck and Sven Porst for reading a draft of the thesis. I am also thankfull to the referee Prof. Thomas Schick for helpfull remarks. Sincere gratitude to my parents for their support.

Last, but not least, I wish to thank Yuriy Holovaty, who inspired my first steps in research, and my school teachers of mathematics Olga Posina and Andriy Dobosevych. 


\section{Contents}

1 Introduction 4

1.1 Problem's origin and survey of literature . . . . . . . . . . . . 4

1.1.1 Classification problem in dimension 4 and Seiberg-Witten equations ................. 4

1.1 .2 Basics of the Seiberg-Witten theory . . . . . . . . . . . 6

1.1.3 Generalized Seiberg-Witten theory . . . . . . . . . . . 10

1.1.4 HyperKähler and quaternionic Kähler geometries . . . 10

1.2 Overview of the thesis . . . . . . . . . . . . . 13

1.3 Addendum: conventions . . . . . . . . . . . . . . . . . . . . 15

1.3 .1 Dirac operator . . . . . . . . . . . . . . . . . . 16

1.3 .2 Seiberg-Witten equations . . . . . . . . . . . . 17

$1.3 .3 \quad$ Seiberg-Witten moduli space . . . . . . . . . . . . . . 18

2 HyperKähler manifolds with hyperKähler potential and triholomorphic $S^{1}$-action 19

$2.1 S^{1}$-Symmetry $\ldots \ldots \ldots \ldots \ldots$

2.1 .1 Induced structure on P . . . . . . . . . . . . . . . 21

2.1 .2 Metric . . . . . . . . . . . . . . . . . . . 22

2.1 .3 Symplectic forms . . . . . . . . . . . . . . . . 23

2.1 .4 Inverse Construction . . . . . . . . . . . . . . . . . . 24

2.1 .5 Quaternionic Flip . . . . . . . . . . . . . . . . . . . . 29

2.2 Examples $\ldots \ldots \ldots \ldots \ldots$

2.3 Indeterminacy of function $v$ : further examples . . . . . . . . . 36

2.4 Kähler structure on $N_{0} \ldots \ldots \ldots \ldots$

\begin{tabular}{|lll}
\hline & Nonlinear Dirac operator and harmonic spinors & 41
\end{tabular}

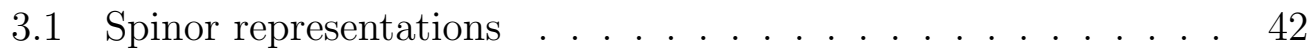

3.2 The Nonlinear Dirac operator . . . . . . . . . . . . . . . . . . . . . . . . . . . . . . . . 50

3.3 Aholomorphic maps . . . . . . . . . . . . . . . . . . . 50

3.3 .1 Linear algebra: aquaternionic operators . . . . . . . . . 50

3.3.2 Aholomorphic maps between hyperKähler manifolds. . 53 
3.3 .3 Aholomorphic sections . . . . . . . . . . . . 54

3.4 Harmonic spinors and aholomorphic sections . . . . . . . . 56

4 Generalized Seiberg-Witten Equations over Kähler Surfaces and Holomorphic Curves $\quad 59$

4.1 Generalized Seiberg-Witten equations . . . . . . . . . . . 60

4.2 Generalized Seiberg-Witten Equations over Kähler Surfaces . . . . . . . . . . . . . . . . . . 60

4.3 Twisted holomorphic curves and solutions to Seiberg-Witten

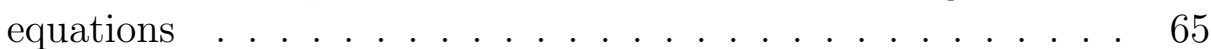

4.4 Seiberg-Witten equations as THCsystem with additional twisting . . . . . . . . . . . 67

4.5 THC equations over products of holomorphic curves . . . . . . 68

4.6 Seiberg-Witten equations over product of holomorphic curves 79

Bibliography $\quad 82$

$\begin{array}{ll}\text { Index } & 88\end{array}$

Curriculum vitae A 


\section{Chapter 1}

\section{Introduction}

\subsection{Problem's origin and survey of literature}

\subsubsection{Classification problem in dimension 4 and Sei- berg-Witten equations}

The problem of classification of smooth manifolds has long been studied. Since Poincaré's time we know that any compact orientable 2-manifold looks like a connected sum of a number of tori (or a sphere):

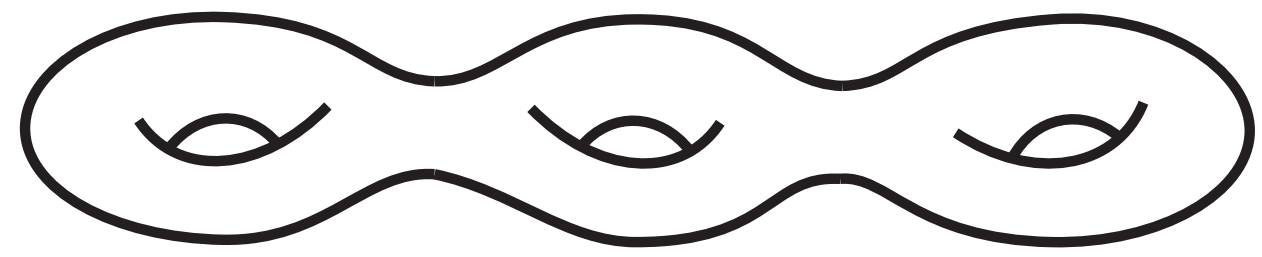

Figure 1.1: Two dimensional manifolds: connected sum of 3 tori.

Since classification of groups, which can occur as the fundamental group of a manifold $M$ with $\operatorname{dim} M \geq 4$, is algorithmically impossible, the classification problem in dimension 4 and greater is algorithmically unsolvable. However, one can try to classify simply connected manifolds instead.

The classification of compact simply connected smooth manifolds in dimension 5 and greater is now also known, however the problem in physically most interesting cases of dimensions 3 and 4 still remains unsolved. First Freedman [27] answered the question when two given 4-manifolds are homeomorphic. Unlike in dimension 2, 4-manifolds can be homeomorphic but not diffeomorphic! It was Donaldson [21] who made the first breakthrough in 
smooth topology in dimension 4 in 1980s. Roughly speaking the idea was the following: if two given homeomorphic 4-manifolds have different spaces

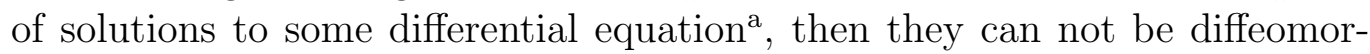
phic. The equation that Donaldson made use of was the celebrated AntiSelf-Duality (ASD for short) equation

$$
F_{a}^{+}=0
$$

where $a$ is a connection on a chosen principal $S U(2)$ bundle and $F_{a}^{+}$denotes the self-dual part of the curvature $F_{a}$. Equation (1.1) has long been known in mathematical physics as Yang-Mills equation. Studying the ASD-equation, Donaldson was able to prove for example the following theorems:

Theorem A. There exist compact topological 4-manifolds, which admit no smooth structure.

Theorem B. There exist compact smooth 4-manifolds, which are homeomorphic but not diffeomorphic.

The nonlinearity of the ASD equation presented serious difficulties and a lot of new techniques had to be developed for its analysis.

Ten years later Seiberg and Witten [62] proposed a system of equations (since then it carries their name) which is now the main tool in 4-manifolds studies (see section 1.3.2 for definition). The roots of the system lie in the depth of the still rather mysterious notion of S-duality in $N=2$ supersymmetric Yang-Mills theory [62, 63].

Nonlinearity of the Seiberg-Witten equations is much milder than the nonlinearity of (1.1). Besides, the gauge group is abelian in Seiberg-Witten theory in contrast to Donaldson theory and therefore the analysis involved was also easier. But the main surprise was that a lot of results obtained via Donaldson theory were much more easily reproved in the realm of SeibergWitten theory.

The most direct application of the Seiberg-Witten invariant $\mathrm{b}^{\mathrm{b}}$ was found by Fintushel and Stern [26]. They constructed an infinite family of manifolds homeomorphic but not diffeomorphic to a K3 surface. Their idea was to perform a surgery along a knot (or link) embedded into a 4-manifold. If we start with a K3 surface, then the resulting manifold can still be homeomorphic to the original surface. However, the Seiberg-Witten invariant of a resulting manifold is described by the Alexander polynomial of the knot

\footnotetext{
${ }^{a}$ it seems inevitable at present that one has to consider nonlinear equations to capture properties of smooth structure.

${ }^{\mathrm{b}}$ certain number derived from the space of solutions to the Seiberg-Witten equations; see definitions 1.1 .5 and 1.1 .6 .
} 
and is different from the Seiberg-Witten invariant of the original K3 surface. Consequently, the original manifold and the one obtained after surgery can not be diffeomorphic.

Shortly after the Seiberg-Witten equations were discovered, other applications were also found. For example, it is interesting to ask whether a smooth manifold admits a metric of positive scalar curvature. It was known, that there are topological obstructions for the existence of a metric with positive scalar curvature: for example, among surfaces only spheres admit such metric. As Witten already remarked, a smooth 4-manifold with positive scalar curvature must have vanishing Seiberg-Witten invariant. Consequently, there is an obstruction to the existence of a metric with positive scalar curvature, which depends on a smooth structure, not just the topological type of a manifold.

Other links with Riemannian geometry have been found by LeBrun [49]. He showed that any Einstein 4-manifold $X$ with non-trivial Seiberg-Witten invariant satisfies the following inequality: $\chi(X)-3 \sigma(X) \geq 0$, where $\chi(X)$ is the Euler characteristic and $\sigma(X)$ is the signature of $X$. This is a restriction on the existence of an Einstein metric.

It was shown by Witten that a connected sum of two 4-manifolds, which both satisfy $b_{2}^{+}>1$, has vanishing Seiberg-Witten invariant [72]. Consequently, a 4-manifold with non-trivial Seiberg-Witten invariant is smoothly indecomposable. On the other hand, Taubes showed [68] that a symplectic 4 -manifold with $b_{2}^{+}>1$ has non-vanishing Seiberg-Witten invariant. Thus, symplectic manifolds with $b_{2}^{+}>1$ are indecomposable.

A powerful tool in symplectic geometry is the Gromov invariant [53]. It is based on counting pseudo-holomorphic curves representing a given homology class. Taubes [67] showed that the Seiberg-Witten and Gromov invariants coincide for compact symplectic manifolds with $b_{2}^{+}>1$. Another relation between solutions of Seiberg-Witten equations and pseudo-holomorphic curves can be found in [17].

\subsubsection{Basics of the Seiberg-Witten theory}

In this section we briefly review the basics of the Seiberg-Witten theory. All statements and constructions are well-known and documented and therefore we will omit proofs and provide only references. For precise definitions and more explanations see section 1.3 . A systematic exposition can be found, for example, in [54, 55, 56, 52].

Let $X$ be a smooth closed orientable 4-manifold with a Riemannian metric $g_{X}$. Choose a $\operatorname{Spin}^{c}(4)$-structure with a determinant line bundle $L$ and let $a$ 
be a connection on $L$. Consequently we get a Dirac operator

$$
\mathcal{D}_{a}: \Gamma\left(W^{+}\right) \longrightarrow \Gamma\left(W^{-}\right),
$$

where $W^{ \pm}$are spinor bundles. Let $\Phi(u)$ denote a self-dual 2-form (see (1.7)) associated to a spinor $u \in \Gamma\left(W^{+}\right)$. The following nonlinear first order partial differential equations for a pair $(a, u)$

$$
\left\{\begin{array}{l}
\mathcal{D}_{a} u=0, \\
F_{a}^{+}+\Phi(u)=0
\end{array}\right.
$$

are called Seiberg-Witten equations.

Theorem 1.1.1. [55] The system (1.2) is elliptic (and hence Fredholm).

A gauge group $\mathcal{G}=\operatorname{Map}\left(X, S^{1}\right)$ acts on solutions (see (1.9p) and we get the associated moduli space $1.10 \mathcal{M}_{S W}$. In general, there is no reason to hope that the moduli space will be nonsingular. However one can achieve smoothness by suitable perturbations.

There are two common ways of perturbations: either one uses parameters that are naturally incorporated into the problem (such as a Riemannian metric in case of the ASD-equation or an almost complex structure in case of the symplectic vortex equation) or perturbs the equations themselves. In the Seiberg-Witten case the latter approach works.

Theorem 1.1.2. [55] Assume $b_{2}^{+}(X)>1$. Then, for a generic self-dual 2form $\eta$, the moduli space $\mathcal{M}_{S W}^{\eta}$ corresponding to the following perturbation of the Seiberg-Witten equations

$$
\left\{\begin{array}{l}
\mathcal{D}_{a} u=0, \\
F_{a}^{+}+\Phi(u)=\eta,
\end{array}\right.
$$

is a nonsingular smooth oriented [ $\square^{\mathrm{C}}$ manifold of dimension

$$
d=\frac{1}{4}\left(c_{1}(L)^{2}-2 \chi(X)-3 \sigma(X)\right)
$$

where $\chi(X)$ and $\sigma(X)$ denote the Euler characteristic and the signature of $X$ correspondingly.

\footnotetext{
${ }^{\mathrm{c}}$ requires certain choices; see [55] for details.
} 
An important property of the Dirac operator is the Weitzenböck formula (see for example [48, 55, 52]), which relates the square of the Dirac operator with the Laplacian of $\nabla^{\hat{a}}$ and components of the curvature $\hat{a}$, where $\hat{a}$ is a connection on spinor bundles determined by $a$ and the Levi-Civita one. In particular, it enables us to obtain a priori bounds for solutions of the Seiberg-Witten equations. In turn, such a priori estimates imply the following compactness result.

Theorem 1.1.3. [55] The Seiberg-Witten moduli space $\mathcal{M}_{S W}^{\eta}$ is compact.

The compactness property is probably the most important feature of the Seiberg-Witten moduli space. It is the main advantage over other similar theories.

Another important consequence of the Weitzenböck formula is that the Seiberg-Witten equations admit variational formulation.

Proposition 1.1.4. [52] Solutions $(a, u)$ of (1.2) are absolute minima of the following functional

$$
S(a, u)=\int_{X}\left(\left|\nabla^{\hat{a}} u\right|^{2}+\left|F_{a}^{+}\right|^{2}+\frac{s}{4}|u|^{2}+\frac{1}{8}|u|^{4}\right) d v l_{X},
$$

where $s$ is a scalar curvature of $X$.

Definition 1.1.5 (Seiberg-Witten invariant: simple case). Pick a $\operatorname{Spin}^{c}(4)$ structure $P_{S p i n^{c}}$ and suppose that $\operatorname{dim} \mathcal{M}_{S W}^{\eta}=0$. It follows from Theorem 1.1.3 that $\mathcal{M}_{S W}^{\eta}$ is a finite collection of points and we define a Seiberg-Witten invariant to be

$$
S W\left(P_{S p i n^{c}}\right)=\sum_{p \in \mathcal{M}_{S W}^{\eta}} \epsilon_{p},
$$

where $\epsilon_{p}= \pm 1$ according to the orientation at the point $p$.

It is a little bit more difficult to define Seiberg-Witten invariant in the general case. First pick a base point $x_{0} \in X$ and consider a subgroup

$$
\mathcal{G}_{0}=\left\{g \in \mathcal{G} \mid g\left(x_{0}\right)=1\right\} \subset \mathcal{G} .
$$

Then the space of solutions to 1.3 modulo $\mathcal{G}_{0}$ is an $S^{1}$ principal bundle over $\mathcal{M}_{S W}^{\eta}$. Let $\mathcal{L} \rightarrow \mathcal{M}_{S W}^{\eta}$ be the associated line bundle.

Definition 1.1.6 (Seiberg-Witten invariant: general case). Let $d=$ $\operatorname{dim} \mathcal{M}_{S W}^{\eta}$. A Seiberg-Witten invariant is the following number

$$
S W\left(P_{\text {Spin }^{c}}\right)=\int_{\mathcal{M}_{S W}^{\eta}} c_{1}(\mathcal{L})^{d / 2}, \quad d \geq 0, d \text { is even }
$$

and $S W\left(P_{\text {Spin }^{c}}\right)=0$ otherwise. 
Denote by $\mathcal{S}(X)$ the set of isomorphism classes of $\operatorname{Spin}^{c}(4)$ structures on $X$.

Theorem 1.1.7. [55] The function

$$
S W: \mathcal{S}(X) \longrightarrow \mathbb{Z}
$$

is well-defined. It is zero on all but finitely many elements of $\mathcal{S}(X)$.

In the remaining part of this section we briefly consider the SeibergWitten equations in the case when the source manifold $X$ is Kähler. Let $K_{X}$ be the canonical line bundle. Pick a $\operatorname{Spin}^{c}(4)$ structure and let $K_{X}^{-1} \otimes L_{0}^{2}$ be its determinant line bundle. Then $W^{+} \cong \Lambda^{0}\left(X ; L_{0}\right) \oplus \Lambda^{0,2}\left(X ; L_{0}\right)$ and hence a spinor $u$ can be written as a pair $u=(\alpha, \beta)$ with $\alpha \in \Omega^{0}\left(X ; L_{0}\right)$ and $\beta \in \Omega^{0,2}\left(X ; L_{0}\right)$.

Theorem 1.1.8. 55] Assume that $X$ is Kähler and let $(a, u)$ be a solution to the Seiberg-Witten equations (1.2). Then the following holds

- connection a defines a holomorphic structure on the determinant line bundle, i.e. $\left(F_{a}\right)^{0,2}=0$;

- $\bar{\alpha} \beta=0$;

- $\alpha$ is a holomorphic section of $L_{0}$ with respect to the holomorphic structure induced by $a$ and $\bar{\beta}$ is a holomorphic section of $K_{X} \otimes L_{0}^{-1}$.

It is sometimes possible to say more about solutions to the Seiberg-Witten equations on Kähler surfaces as well as about the invariant.

Proposition 1.1.9. [55] Let $X$ be a Kähler surface.

- If $\operatorname{deg} K_{X}<0$, then the only solutions to Seiberg-Witten equation are reducible.

- Suppose $\operatorname{deg} K_{X}>0$. Then $S W\left(P_{K_{X}^{-1}}\right)=1$, where $P_{K_{X}^{-1}}$ denotes a $\operatorname{Spin}^{c}(4)$ structure, whose determinant line bundle is $K_{X}^{-1}$.

Finally we would like to note that it is possible to compute the SeibergWitten invariant for minimal algebraic surfaces of general type, which happens to be non-trivial. Similar results for symplectic manifolds were obtained by Taubes [66, 67, 68]. 


\subsubsection{Generalized Seiberg-Witten theory}

In the recent paper [59] V. Pidstrigach proposed a certain generalization of the Seiberg-Witten equations (1.2). The basic idea was to replace the spinor vector bundle by a certain fibre bundle with a nonlinear fibre. More precisely, a fibre of the spinor bundle $W^{+}$can be identified with the simplest hyperKähler manifold $\mathbb{H} \cong \mathbb{C}^{2}$; then one replaces $\mathbb{H}$ with an arbitrary hyperKähler manifold $M$ with certain symmetries. It is then possible to define a Dirac operator, which acts on sections of the "nonlinear spinor bundle". Such generalizations of the Dirac operator were already known to physicists [3, 6]. This idea was also described in [69] for 3-dimensional manifolds.

Further, given a spinor $u$, i.e. a section of nonlinear spinor bundle, it is possible to construct a self-dual 2-form $\Phi(u)$ with the help of a hyperKähler momentum map. Hence, the generalization of the Seiberg-Witten equations can be written in the same form as (1.2).

It was shown in 59] that a variant of the Weitzenböck formula also holds for the above mentioned generalized Dirac operator. This is used to show that the moduli space associated to the generalized Seiberg-Witten equations is compact for the abelian gauge group provided the fibre $M$ admits a hyperKähler potential, i.e. a single function, which is a Kähler potential for all complex structures simultaneously.

\subsubsection{HyperKähler and quaternionic Kähler geometries}

The previous section motivates our interest in hyperKähler manifolds and closely related to them quaternionic Kähler ones. Their study is very interesting on its own and there are several reasons for that. As it was shown by Berger [8] there are very few groups, which can occur as a holonomy group of a simply connected nonsymmetric locally irreducible Riemannian manifold. In particular, Berger's list contains $S p(n)$ and $S p(n) S p(1)$ : the first is a holonomy group of a hyperKähler manifold while the second of a quaternionic Kähler one. It means that hyper- and quaternionic-Kähler manifolds are examples of basic Riemannian geometries. One can describe hyperKähler manifolds in other words as follows.

Definition 1.1.10. A Riemannian manifold $(M, g)$ is hyperKähler if it admits three covariantly constant complex structures $I_{r}, r=1,2,3$ with quaternionic relations

$$
I_{1} I_{2}=-I_{2} I_{1}=I_{3}, \quad I_{r}^{2}=-i d,
$$

compatible with Riemannian structure: $g\left(I_{r} \cdot, I_{r} \cdot\right)=g(\cdot, \cdot)$. 
Hitchin [38 proved in 1987 extremely useful criteria of integrability of complex structures: $I_{r}$ are covariantly constant iff 2 -forms $\omega_{r}(\cdot, \cdot)=g\left(\cdot, I_{r} \cdot\right)$ are symplectic (i.e. closed). ometimes it is convenient to consider all three symplectic forms together as one $\operatorname{Im} \mathbb{H}$-valued 2-form:

$$
\omega=\omega_{1} i+\omega_{2} j+\omega_{3} k .
$$

In contrast to hyperKähler manifolds, a quaternionic Kähler manifold $N$ admits complex structures (and correspondingly 2-forms $\omega_{r}$ ) only locally. Nevertheless the 4 -form $\Omega=w_{1} \wedge \omega_{1}+w_{2} \wedge \omega_{2}+w_{3} \wedge \omega_{3}$, called a fundamental 4-form, exists globally and determines the quaternionic Kähler structure. In this case the integrability means that the fundamental 4-form is covariantly constant and this is equivalent to $d \Omega=0$ provided $\operatorname{dim} N \geq 12$. In dimension 4 quaternionic Kähler by definition means Einstein and self-dual.

Another source of interest to hyper- and quaternionic-Kähler manifolds is physics. It is well known 61] that both classes of manifolds are automatically Einstein and therefore they are of particular interest to physicists. On the other hand, such manifolds naturally appear in the supersymmetric $\sigma$-model [2, 3, 6].

One of the major problems of quaternionic Riemannian geometry is to construct examples of hyper- and quaternionic-Kähler manifolds. It is a sharp contrast to complex geometry, where there are no problems to construct Kähler manifolds.

The first nontrivial example of a hyperKähler metric was one constructed by Eguchi and Hanson [23] on $T^{*} \mathbb{C P}^{1}$, which was lately generalised by Calabi [13] to all $T^{*} \mathbb{C P}^{n}$. In dimension four Gibbons and Hawking [34] described all hyperKähler manifolds with $S^{1}$-symmetry (multiinstanton metrics in physical terminology; see example 2.2 .4 for details).

The basic examples of compact hyperKähler manifolds are flat torii and K3 surfaces. These are in fact the only compact hyperKähler 4-manifolds. The existence of a hyperKähler metric on a K3 surface follows from the Yau's proof of the Calabi conjecture [74, 75]. For a more explicit construction, see [50].

Another useful source of hyperKähler manifolds is (finite- and infinitedimensional) hyperKähler reduction [36]: if a group $G$ acts isometrically and triholomorphically on a hyperKähler manifold $M$ with momentum map $\mu$ : $M \longrightarrow \mathfrak{g}^{*} \otimes \operatorname{Im} \mathbb{H}$ and $a \in \mathfrak{z} \otimes \operatorname{Im} \mathbb{H}$ is a regular value of $\mu$, then $\mu^{-1}(a) / G$ inherits hyperKähler structure.

One of the most important features about hyperKähler structures is that many natural spaces occurring in gauge theory possess such structure. The key point is that very often such spaces can be viewed as infinite-dimensional 
hyperKähler reductions. Among examples are self-dual instantons over $\mathbb{R}^{4}$ [51] or more generally over a 4-dimensional hyperKähler manifold [41, 47] and closely related spaces: monopoles [19, 5, 39, 9], solutions to the self-duality equations over a Riemann surface [38] and Nahm moduli spaces [19, 45]. Coadjoint orbits of complex semisimple Lie groups [46, 44] form a particularly interesting class of hyperKähler manifolds. In fact, the hyperKähler structure on coadjoint orbits is obtained representing them as suitable Nahm moduli spaces.

Concerning quaternionic Kähler manifolds, their geometry strongly depends on the sign of the scalar curvature $s$, which is automatically constant. In particular, if $s=0$ the manifold is locally hyperKähler and therefore there is a strong tendency to include $s \neq 0$ in the definition of quaternionic Kähler manifolds. We are mainly interested in positive quaternionic Kähler manifolds and consider very briefly some of their properties below.

We would like to point out that quaternionic Kähler manifold $N$ is not Kähler unless $s=0$. Moreover, a positive quaternionic Kähler manifold does not even admit a compatible almost complex structure [1].

On the other hand, the quaternionic structure of $N$ can always be untwisted by passing to the total space of a suitable fibre bundle. In particular, Swann shows [65] that for a quaternionic Kähler manifold $N$ with positive scalar curvature there exists a hyperKähler manifold $\mathcal{U}(N)$, which admits a hyperKähler potential (a single function which is a Kähler potential for all complex structures simultaneously). On the other hand, to any hyperKähler manifold with hyperKähler potential one can associate a positive quaternionic Kähler one. Note that the existence of a hyperKähler potential was required to get a compactness property of the moduli space associated to the generalized Seiberg-Witten equations (see section 1.1.3).

All positive symmetric quaternionic Kähler manifolds were classified in [73] and they are called "Wolf spaces". They are the only known examples of complete positive quaternionic Kähler manifolds. For example, in dimension 4 there are only two such manifolds: $\mathbb{H} \mathbb{P}^{1} \cong S^{4}$ and $\mathbb{C P}^{2}$. However, it is no longer true if one considers orbifolds instead of manifolds: correspondent examples were constructed by Galicki and Lawson [32] with the help of quaternionic Kähler reduction method developed in the same paper.

It is completely clear that neither this section nor Section 1.1.1 provide complete overview of the corresponding subjects and it was definitely not the author's aim. My best hope was to indicate some important recent developments in the corresponding areas related to the research of this thesis. 


\subsection{Overview of the thesis}

The central aim of my research presented in this thesis was the study of the generalized Seiberg-Witten equations (see section 1.1.3). The problem involves certain objects, although defined earlier but studied from a different perspective, such as nonlinear Dirac operator or hyperKähler manifolds with prescribed symmetries. To get a better insight into the main problem we study in the first two chapters their properties alone. The last chapter is devoted to the generalized Seiberg-Witten equations.

HyperKähler geometry. As we have already remarked the moduli space of the generalized Seiberg-Witten equations is compact provided the target hyperKähler manifold $M$ admits a hyperKähler potential. In order to formulate the equations it is necessary that $M$ also admits a triholomorphic action of a Lie group $G$ (see [59] for details). In this thesis only the simplest case $G=S^{1}$ will be considered. Natural questions arise: how many such manifolds do we know? what are their properties? These questions are addressed in Chapter 2

A partial answer to the above questions is contained in Swann's paper [65]. Namely, Swann shows that to each hyperKähler manifold with a hyperKähler potential and $G$-symmetry corresponds a quaternionic Kähler manifold with positive scalar curvature also carrying a $G$-symmetry and vice versa. The problem is that we know only a few examples of quaternionic Kähler manifolds with positive scalar curvature.

In Chapter 2 we obtain a classification of all hyperKähler manifolds $M$ with a hyperKähler potential and additional triholomorphic and isometric action of $S^{1}$. It turns out, that such manifolds can be reconstructed by their hyperKähler reductions $\tilde{M}$ with respect to a nonzero value of momentum map. The main result of the chapter is Theorem 2.1.3, which describes how to construct $M$ as well as its hyperKähler structure by a given $\tilde{M}$. This construction can be viewed as an inverse one to the hyperKähler reduction. Moreover, the hyperKähler structure of $M$ is described quite explicitly which allows to obtain not only existence results, but metric and symplectic forms themselves. Dividing such manifolds by $\mathbb{H}^{*}$ as described in [65] we obtain quaternionic Kähler manifolds with $S^{1}$-symmetry (Theorem 2.1.8). In this way we get new examples of hyper- and quaternionic- Kähler manifolds (see Section 2.3). We prove for example that the total spaces of $\mathcal{O}_{\mathbb{P}^{1}}(m), m \geq 1$ admit an Einstein and self-dual metric with positive scalar curvature. On the other hand we obtain interesting relations between known manifolds. For example, it turns out that Gibbons-Hawking spaces are closely related to nilpotent orbits (see example 2.2.4). 
Chapter 2 is self-contained and can be regarded as a contribution to the hyper- and quaternionic-Kähler geometry.

Nonlinear Dirac operator. In Chapter 3 we study properties of the generalized Dirac operator acting on sections of a certain fibre bundle, where fibre is a hyperKähler manifold with certain symmetries. We show that the Dirac operator $\mathcal{D}$ can be regarded as an analogue of $\partial$-operator in complex geometry. Similarly, harmonic spinors, i.e. solutions of the equation $\mathcal{D} u=0$, can be regarded as an analogue of (anti)holomorphic sections in complex context.

Consider for simplicity a case when the source 4-dimensional manifold $X$ is itself hyperKähler with complex structures $\left(J_{1}, J_{2}, J_{3}\right)$, while $\left(I_{1}, I_{2}, I_{3}\right)$ denote the complex structures of $M$. Then harmonic spinors can be viewed as maps $u: X \rightarrow M$ satisfying the quaternionic analogue (3.15) of the Cauchy-Riemann equation. We show in Proposition 3.3.10 that maps satisfying (3.15), i.e. which are believed to be a "right" analogue of holomorphic maps in quaternionic context, are exactly those, whose differential has no triholomorphic component (meaning "commuting with all 3 complex structures"). Hence we propose the term "aholomorphic" for such maps. We also extend the notion of aholomorphicity into quaternionic Kähler context via the Swann construction [65].

Generalized Seiberg-Witten equations over Kähler surfaces. In the last chapter we study the generalized Seiberg-Witten equations over Kähler surfaces as the title suggests. In particular we prove an analogue of Theorem 1.1.8 and consider connections of the generalized Seiberg-Witten equations over Kähler surfaces with other different gauge theories studied in [40, 18, 58]. We consider THC-system defined in [40] (see also 44.8) over the product of Riemann surfaces as a prototype of generalized Seiberg-Witten equations. We show (modulo adiabatic limit conjecture) in Theorem 4.5.6 that in the limit as a metric over one of the curves becomes very large solutions of the THC-system become holomorphic curves in the moduli space of the symplectic vortex equations [18] over the other curve. Then we show (also modulo adiabatic limit conjecture) that analogous result holds for the generalized Seiberg-Witten equations (see theorem 4.6.3).

The main results of the thesis were presented on the Research seminars of Prof.V.Pidstrygach, Göttingen 2002-2005; Summer School Algebraic Groups, Göttingen June-July, 2005; Symposium Geometry, Conformal Field Theory and String Theory, Durham, United Kingdom, July 2005; Seminar Gauge theory and Topology of the Sonderforschungsbereich 701, Bielefeld, October 2005 . 


\subsection{Addendum: conventions}

Quaternions and related groups. By $\mathbb{H}$ we denote the 4-dimensional $\mathbb{R}$-algebra of quaternions with the basis $(1, i, j, k)$ such that

$$
i^{2}=j^{2}=k^{2}=-1, \quad i j=-j i=k .
$$

It follows that any quaternion can be written as $h=h_{0}+h_{1} i+h_{2} j+h_{3} k$ with real numbers $h_{l}$. Sometimes it is convenient to decompose $\mathbb{H}=\mathbb{C} \oplus j \mathbb{C} \cong \mathbb{C}^{2}$ and we write $h=a+j b$, where $a, b \in \mathbb{C}, a=h_{0}+h_{1} i, b=h_{2}-h_{3} i$. Certainly, one may also decompose $\mathbb{H}=\mathbb{C} \oplus \mathbb{C} j$, however the previous decomposition better suits our further conventions. The function $\|\cdot\|: \mathbb{H} \rightarrow \mathbb{R}_{+}$,

$$
\|h\|^{2}=\sum_{l=0}^{4} h_{l}^{2}=|a|^{2}+|b|^{2}
$$

turns $\mathbb{H}$ into the normed space. Further, by the real part of $h$ we mean $h_{0} \in \mathbb{R}$ and by its imaginary part the quaternion $h_{1} i+h_{2} j+h_{3} k$. Consequently we have one more decomposition $\mathbb{H}=\mathbb{R} \oplus \operatorname{Im} \mathbb{H}$, which defines a quaternionic conjugation (or, simply conjugation): $\bar{h}=\operatorname{Re} h-\operatorname{Im} h$.

By $\mathbb{H}^{*}$ we will denote the group of invertible quaternions. A quaternionic unitary group $S p(1)$ is defined as the subgroup of $\mathbb{H}^{*}$ consisting of quaternions of unitary length:

$$
S p(1)=\{q \in \mathbb{H}:|q|=1\} .
$$

Observe that the group $S p(1)$ is isomorphic to $S U(2)$. Indeed, recall that $\mathbb{H} \cong \mathbb{C}^{2}$. Then the isomorphism is given via the map $q \mapsto A_{q}: \mathbb{H} \rightarrow \mathbb{H}$, $A_{q} h=q h$.

Further, the group $\operatorname{Spin}(4)$ is a product of two copies of $S p(1)$ :

$$
\operatorname{Spin}(4)=S p_{+}(1) \times S p_{-}(1),
$$

where we use subscripts " \pm " to distinguish between different copies. Notice that $\operatorname{Spin}(4)$ is a double cover of $S O(4)$ and the isomorphism $\operatorname{Spin}(4) / \pm 1 \cong$ $S O(4)$ is given by

$$
\left(q_{+}, q_{-}\right) \mapsto B_{q_{+}, q_{-}}: \mathbb{H} \rightarrow \mathbb{H}, \quad B_{q_{+}, q_{-}} h=q_{-} h \bar{q}_{+}
$$

and we also take the obvious identification $\mathbb{H} \cong \mathbb{R}^{4}$ into account.

Finally, we define

$$
\operatorname{Spin}^{c}(4)=\operatorname{Spin}(4) \times_{\{ \pm 1\}} S^{1}=\left\{\left[q_{+}, q_{-}, \lambda\right]: q_{ \pm} \in S p_{ \pm}(1), \lambda \in S^{1}\right\} .
$$


Notice that $\operatorname{Spin}^{c}(4) / S^{1} \cong S O(4)$. Hence, the representation of $\operatorname{Spin}^{c}(4)$, which induces a standard representation of $S O(4)$ is given by

$$
\left[q_{+}, q_{-}, \lambda\right] \mapsto B_{\left[q_{+}, q_{-}, \lambda\right]}^{c}: \mathbb{H} \rightarrow \mathbb{H}, \quad B_{\left[q_{+}, q_{-}, \lambda\right]}^{c} h=q_{-} h \bar{q}_{+} .
$$

Spinor Representations. By $W$ we mean the standard $S U(2)-$ representation on $\mathbb{C}^{2}$ or equivalently $S p(1)$-representation on $\mathbb{H}$ by multiplication on the left. Further $W^{ \pm}$denote $\operatorname{Spin}{ }^{c}(4)$-representations on $\mathbb{H}$ given by

$$
\left(\left[q_{+}, q_{-}, \lambda\right], h\right) \mapsto q_{ \pm} h \lambda
$$

and $\mathbb{R}^{4}$ denotes representation of the same group on the same space given by $\left(\left[q_{+}, q_{-}, \lambda\right], h\right) \mapsto q_{-} h \bar{q}_{+}$.

A Clifford multiplication is a homomorphism of $\operatorname{Spin}^{c}(4)$ representations

$$
\mathbb{R}^{4} \otimes W^{+} \longrightarrow W^{-}
$$

defined by the formula

$$
h_{1} \otimes h_{2} \mapsto h_{1} h_{2}
$$

\subsubsection{Dirac operator}

Let $X^{4}$ be a closed oriented Riemannian manifold and $P_{s o}$ be its principal $S O(4)$ bundle of orthonormal oriented frames. A $\operatorname{Spin}^{c}(4)$ principal bundle $P_{\text {Spinc }}$ is called a lifting of $P_{\text {so }}$ if $P_{\text {Spin }^{c}} / S^{1} \cong P_{\text {so }}$, where $S^{1} \subset \operatorname{Spin}^{c}(4)$ is a standard embedding.

Proposition 1.3.1. [55] For any oriented 4-manifold $X$ the principal $S O(4)$ bundle $P_{\text {so }}$ of orthonormal oriented frames can be lifted to Spinc ${ }^{c}(4)$ structure $P_{\text {Spin }^{c} \text {. }}$

Now pick any $\operatorname{Spin}^{c}(4)$ principal bundle $P_{\text {Spin }^{c}}$ as in the above proposition. If we define the "determinant representation" $L$ of $\operatorname{Spin}^{c}(4)$ to be

$$
\rho_{\text {det }}:\left(\left[q_{+}, q_{-}, \lambda\right], z\right) \mapsto \lambda^{2} z, \quad z \in \mathbb{C}
$$

then we get a corresponding Hermitian line bundled $\AA^{\mathrm{d}} L=P_{\operatorname{Spin}^{c}} \times_{\operatorname{Spin}^{c}(4), \rho_{\text {det }}} \mathbb{C}$ called the determinant line bundle. If $H^{2}(X, \mathbb{Z})$ has no torsion, then the lifting $P_{\text {Spin }^{c}}$ is uniquely determined by the Chern class of the determinant line bundle $c_{1}(L) \in H^{2}(X, \mathbb{Z})$.

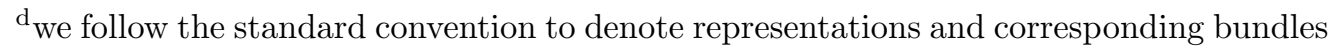
by the same letters.
} 
The representations (1.5) give rise to the corresponding vector bundles $W^{ \pm}$. It follows from (1.6) that we have a homomorphism of vector bundles $T X \otimes W^{+} \rightarrow W^{-}$. The Riemannian metric of $X$ provides an isomorphism $T X \cong T^{*} X$ and therefore we have a map

$$
C l: T^{*} X \otimes W^{+} \longrightarrow W^{-}
$$

also called a Clifford multiplication.

Further, a choice of connection $a$ on $P_{\text {det }}=P_{\text {Spin }} / \operatorname{Spin}(4)$ together with the Levi-Civita connection on $P_{\text {so }}$ determines a connection $\hat{a}$ on $P_{\text {Spin }}$. The connection $a$ can be equivalently viewed as a Hermitian connection on the determinant line bundle $L$. Thus we have a covariant derivative

$$
\nabla^{\hat{a}}: \Gamma\left(W^{+}\right) \longrightarrow \Gamma\left(W^{-}\right) .
$$

Definition 1.3.2 (Dirac operator). The first order differential operator given by the sequence

$$
\mathcal{D}_{a}: \Gamma\left(W^{+}\right) \stackrel{\nabla^{\hat{a}}}{\longrightarrow} \Gamma\left(T^{*} X\right) \otimes \Gamma\left(W^{+}\right) \stackrel{C l}{\longrightarrow} \Gamma\left(W^{-}\right)
$$

is called the Dirac operator.

\subsubsection{Seiberg-Witten equations}

Let $\mathbb{R}_{+}^{3}$ denote the standard representation of $S O_{+}(3)$. If we identify $\mathbb{R}^{3}$ with $\operatorname{Im} \mathbb{H}$, then the induced representation of $\operatorname{Spin}^{c}(4)$ in quaternionic notations can be written as $\left(\left[q_{+}, q_{-}, \lambda\right], h\right) \mapsto q_{+} h \bar{q}_{+}, h \in \operatorname{Im} \mathbb{H}$. Consider the following nonlinear equivariant map

$$
\Phi: W^{+} \longrightarrow \mathbb{R}_{+}^{3} \quad \Phi(h)=h i \bar{h} .
$$

It follows that we obtain the corresponding nonlinear map from the positive spinor bundle into the bundle of self-dual 2-forms on $X$ :

$$
\Phi: W^{+} \longrightarrow \Omega_{+}^{2}(X) .
$$

Definition 1.3.3 (Seiberg-Witten equations). The system of partial differential equations

$$
\left\{\begin{array}{l}
\mathcal{D}_{a} u=0, \\
F_{a}^{+}+\Phi(u)=0
\end{array}\right.
$$

where unknowns are a connection $a$ and spinor $u$ is called the Seiberg-Witten equations. 


\subsubsection{Seiberg-Witten moduli space}

Let $\mathcal{A}\left(P_{\text {det }}\right)$ denote the space of connections on the principal bundle $P_{\text {det }}$. A gauge group $\mathcal{G}=\operatorname{Map}\left(X, S^{1}\right)$ of smooth maps from $X$ into $S^{1}$ acts on pairs $(a, u) \in \mathcal{A}\left(P_{\text {det }}\right) \times \Gamma\left(W^{+}\right):$

$$
g \cdot(a, u)=\left(a-2 g^{-1} d g, g u\right), \quad g \in \mathcal{G} .
$$

The above defined action of the gauge group induces action on the space of solutions to the Seiberg-Witten equations [55]. It is clear that the action is free provided $u \not \equiv 0$. By a suitable perturbation of equations one can achieve [55] that $(a, 0)$ is not a solution of the Seiberg-Witten equations if $b_{2}^{+}>1$.

Definition 1.3.4. The quotient space

$$
\left.\mathcal{M}_{S W}=\left\{(a, u) \in \mathcal{A}\left(P_{\text {det }}\right) \times \Gamma\left(W^{+}\right) \mid(a, u) \text { solves } 1.8\right)\right\} / \mathcal{G}
$$

is called a (Seiberg-Witten) moduli space. 


\section{Chapter 2}

\section{HyperKähler manifolds with hyperKähler potential and triholomorphic $S^{1}$-action}

In order to define a nonlinear generalization of the Dirac operator (see Section 3.2), the target hyperKähler manifold $\left(M, g, I_{1}, I_{2}, I_{3}\right)$ must admit certain symmetries. The most important ingredient is a so-called permuting action of $S p(1)$ (or $S O(3))$.

Definition 2.0.5. An isometric action of the group $S p(1)$ is called permuting if the following holds

$$
\mathcal{L}_{\zeta} \omega=[\zeta, \omega] \quad \Longleftrightarrow \quad\left(L_{q}\right)^{*} \omega=q \omega \bar{q}, \quad q \in S p(1),
$$

where $\zeta \in \operatorname{Im} \mathbb{H} \cong \mathfrak{s p}(1)$ and $\omega=\omega_{1} i+\omega_{2} j+\omega_{3} k$ is the quaternion-valued 2-form with $\omega_{l}(\cdot, \cdot)=g\left(\cdot, I_{l} \cdot\right)$.

We will also say, that $S p(1)$ with the above property permutes complex structures of $M$.

Swann 65] shows that permuting action of $S p(1)$ can be extended to homothetic action of the whole $\mathbb{H}^{*}=\mathbb{R}_{+}^{*} \times S p(1)$ if the vector field $I Y_{I}$ is independent of a complex structure $I$, where $Y_{I}$ is a Killing vector field of $S^{1} \subset S p(1)$ which preserves $I$. In particular

$$
I_{1} Y_{1}=I_{2} Y_{2}=I_{3} Y_{3}=-Y_{0}
$$

where we put $Y_{r}=Y_{I_{r}}$ for short and a vector field $Y_{0}$ generates homothetic action of $\mathbb{R}_{+}^{*} \subset \mathbb{H}^{*}:\left(L_{r}\right)^{*} g=r^{2} g$. We will also call such $\mathbb{H}^{*}$-action permuting. Under these circumstances $N=M / \mathbb{H}^{*}$ has positive scalar curvature and carries a quaternionic Kähler structure. 
On the other hand, for any quaternionic Kähler manifold $N$ with positive scalar curvature Swann constructs a hyperKähler manifold $\mathcal{U}(N)$ which enjoys permuting action of $\mathbb{H}^{*}$. Such manifolds are also distinguished by the property of carrying a hyperKähler potential, i.e. function $\rho: M \longrightarrow \mathbb{R}$ which is Kähler potential for each complex structure simultaneously.

It was shown in [59] that the Seiberg-Witten moduli space associated to the generalized Seiberg-Witten equations is compact provided $M$ admits a permuting $\mathbb{H}^{*}$ action or, equivalently, a hyperKähler potential.

In this section we study hyperKähler manifolds $M$ with hyperKähler potential and triholomorphic action of $S^{1}$. The triholomorphic action of $S^{1}$ is necessary for the definition of the Seiberg-Witten equations. First we show how to reconstruct the manifold $M$ by its hyperKähler reduction $\tilde{M}$ with respect to a nonzero value of momentum map. This is done in Theorem 2.1.3. which simultaneously provides a classification of hyperKähler manifolds with hyperKähler potential and triholomorphic action of $S^{1}$. Then we describe correspondent quaternionic Kähler manifolds (see Theorem 2.1.8). In Section 2.3 we describe new examples of hyper- and quaternionic-Kahler manifolds making use of a certain freedom in choice of parameters of the construction. We prove for example that the total spaces of $\mathcal{O}_{\mathbb{P}^{1}}(m), m \geq 1$ admit an Einstein and self-dual metric with positive scalar curvature. The last section is devoted to Kähler structure on quaternionic Kähler manifolds with positive scalar curvature naturally invoked by the action of $S^{1}$. Theorem 2.4.1 was known earlier from twistor theory, however our proof is new. We not only prove the existence of Kähler structure, but also compute it explicitly.

\section{$2.1 \quad S^{1}$-Symmetry}

Let $M$ be a hyperKähler manifold with $\mathbb{H}^{*}$-action permuting complex structures. Suppose also that $M$ admits a hyperKähler action of $S^{1}$ (which we prefer to denote $S_{0}^{1}$ in order to distinguish this group from another one, which will appear later and is also isomorphic to $S^{1}$ ) with momentum map $\mu: M \longrightarrow \operatorname{Im} \mathbb{H}$,

$$
d \mu=-\imath_{K_{0}} \omega,
$$

where $K_{0}$ is a Killing vector field of $S_{0}^{1}$. We assume also that these two actions commute. It follows that

$$
\mu \circ L_{x}=x \mu \bar{x}, \quad x \in \mathbb{H}^{*} .
$$

Now fix an imaginary quaternion, say $i$, and consider corresponding level set $P=\mu^{-1}(i)$. Since $x m=L_{x} m \in \mu^{-1}(x i \bar{x})$ and $\mathbb{H}^{*}$ acts transitively on 
$\operatorname{Im} \mathbb{H} \backslash\{0\}$ the map

$$
f: \mathbb{H}^{*} \times P \longrightarrow M \backslash \mu^{-1}(0), \quad(x, m) \mapsto x m,
$$

is surjective. Notice that

$$
M_{0}=M \backslash \mu^{-1}(0)
$$

is open and everywhere dense submanifold of $M$.

However the map $f$ is not injective. Indeed $\mu^{-1}(i)=P$ inherits action of $\operatorname{Stab}_{i}=S^{1} \subset \mathbb{H}^{*}$ (to which we now give a label $S_{r}^{1}$ ) and it follows that points $(x, m)$ and $(x z, \bar{z} m), z \in S_{r}^{1}$ are mapped into the same point $x m$ in $M_{0}$. Thus the manifold $M_{0}$ can be described as $\mathbb{H}^{*} \times_{S_{r}^{1}} P$. Now the challenge is to express the hyperKähler structure of $M_{0}$ in terms of its "components" $\mathbb{H}^{*}$ and $P$.

While the first "component" $\mathbb{H}^{*}$ is quite easy the second one needs to be understood more deeply for the future purposes.

\subsubsection{Induced structure on $\mathrm{P}$}

First observe that $\tilde{M}:=P / S_{0}^{1}$ is just a hyperKähler reduction of $M$ and therefore is itself a hyperKähler manifold. Here we assume that $i$ is a regular value of the momentum map. Thus $P$ can be thought of as $S_{0}^{1}$-principal bundle over $\tilde{M}$. Moreover it comes equipped with a connection

$$
\xi(\cdot)=v g\left(K_{0}, \cdot\right) \in \Omega^{1}(P),
$$

where $v^{-1}=g\left(K_{0}, K_{0}\right), v: \tilde{M} \longrightarrow \mathbb{R}_{>0}$. Notice that the induced metric $\tilde{g}$ on $\tilde{M}$, the connection $\xi$ and the function $v$ together determine the metric on $P$ since $T . P \cong \mathbb{R} K_{0} \oplus T . \tilde{M}$ :

$$
g_{\mathrm{P}}=\tilde{g}+v^{-1} \xi^{2} .
$$

The connection $\xi$ defines a horizontal lift $\hat{\mathrm{u}} \in T P$ of a tangent vector $\mathrm{u} \in T \tilde{M}$.

As we have already remarked $P$ inherits the action of $S_{r}^{1}$, which descends to $\tilde{M}$. The latter action has a nice property (inherited from $M$ ) of fixing complex structure $I_{1}$ and rotating the plane spanned by $I_{2}$ and $I_{3}$. Denote by $K_{r}$ a Killing vector field of $S_{r}^{1}$-action on $\tilde{M}$ and by $w$ the squared norm of $K_{r}$ :

$$
w: \tilde{M} \rightarrow \mathbb{R}_{>0}, \quad w=\left\|K_{r}\right\|^{2} .
$$

Below we will also use a quaternion-valued 1-form $\eta$ generated by $K_{r}$ :

$$
\eta=\imath_{K_{r}} \tilde{g}+\imath_{K_{r}} \tilde{\omega} \in \Omega^{1}(\tilde{M} ; \mathbb{H}) .
$$

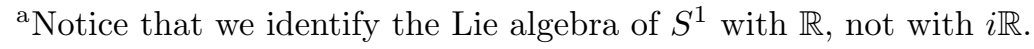


Further, recall that $Y_{1}$ is the Killing vector field of the $S_{r}^{1}$-action on $P$. Then $Y_{1}$ and $K_{r}$ are related as follows. First observe that T. $M=$ T.P甲 $\mathbb{R} I_{1} K_{0} \oplus \mathbb{R} I_{2} K_{0} \oplus \mathbb{R} I_{3} K_{0}$ and one also has

$$
\text { T. } P=\operatorname{Ker} \mu_{*}, \quad \mu_{*} I_{1} K_{0}=v^{-1} i, \mu_{*} I_{2} K_{0}=v^{-1} j \quad \text { and } \quad \mu_{*} I_{3} K_{0}=v^{-1} k .
$$

Now taking $x=\exp (i t)$ in formula $(2.3)$ and differentiating with respect to $t$ one obtains that the formula $Y_{1}=\hat{K}_{r}+a K_{0}$ holds on $P$. The same argument gives that $\mu_{*} Y_{0}=2 i$ or in other words $Y_{0}=\hat{Y}^{\prime}+b K_{0}+2 v I_{1} K_{0}$. It follows from the equation $I_{1} Y_{0}=Y_{1}$ that $Y^{\prime}=-I_{1} K_{r}, b=0, a=-2 v$. Summing up we obtain

$$
\begin{gathered}
Y_{0}=-I_{1} \hat{K}_{r}-2 v I_{1} K_{0}, \quad Y_{1}=\hat{K}_{r}+2 v K_{0}, \\
Y_{2}=I_{3} \hat{K}_{r}+2 v I_{3} K_{0}, \quad Y_{3}=-I_{2} \hat{K}_{r}-2 v I_{2} K_{0} .
\end{gathered}
$$

Remark 2.1.1. Since actions of $S_{0}^{1}$ and $S_{r}^{1} \subset \mathbb{H}^{*}$ commute, it follows that the connection $\xi$ enjoys additional property of being $S_{r}^{1}$ invariant. On infinitesimal level this means that $0=\mathcal{L}_{Y_{1}} \xi=\imath_{Y_{1}} d \xi+d \imath_{Y_{1}} \xi=\imath_{K_{r}} F_{\xi}+2 d v$, where $F_{\xi} \in \Omega^{1}(\tilde{M})$ denotes the curvature form of $\xi$. Thus, invariance of $\xi$ with respect to action of $S_{r}^{1}$ on $P$ is equivalent to

$$
\imath_{K_{r}} F_{\xi}+2 d v=0 .
$$

Note also that the function $v$ is $S_{r}^{1}$-invariant by the same reason.

\subsubsection{Metric}

Since $M$ is a Riemannian manifold the map $f$, defined by (2.4), induces a metric $f^{*} g$ on $\mathbb{H}^{*} \times P$. Notice that since $f$ is not injective, this metric degenerates on tangent vectors to fibres. Our next aim is to calculate $f^{*} g$ explicitly in terms of tensors on $\mathbb{H}^{*}$ and $\tilde{M}$ as well as the connection $\xi$ and function $v$.

Let $(x, m) \in \mathbb{H}^{*} \times P$ and $\left(h_{1}, \mathrm{v}_{1}\right),\left(h_{2}, \mathrm{v}_{2}\right) \in T_{x} \mathbb{H}^{*} \times T_{m} P$. Put also $\alpha=x^{-1} h_{1}, \beta=x^{-1} h_{2} \in T_{1} \mathbb{H}^{*}$ and denote by $\mathrm{Y}_{\alpha}$ and $\mathrm{Y}_{\beta}$ the Killing vector fields of $\mathbb{H}^{*}$-action at the point $m$ corresponding to the Lie algebra elements $\alpha$ and $\beta$. Obviously $\left(\mathrm{Y}_{1}, \mathrm{Y}_{i}, \mathrm{Y}_{j}, \mathrm{Y}_{k}\right)=\left(Y_{0}, Y_{1}, Y_{2}, Y_{3}\right)$. Further, one has

$$
\begin{aligned}
f^{*} g\left(\left(h_{1}, \mathrm{v}_{1}\right),\left(h_{2}, \mathrm{v}_{2}\right)\right) & =g\left(\left(L_{x}\right)_{*}\left(\mathrm{Y}_{\alpha}+\mathrm{v}_{1}\right),\left(L_{x}\right)_{*}\left(\mathrm{Y}_{\beta}+\mathrm{v}_{2}\right)\right)= \\
& =|x|^{2} g\left(\mathrm{Y}_{\alpha}+\mathrm{v}_{1}, \mathrm{Y}_{\beta}+\mathrm{v}_{2}\right) .
\end{aligned}
$$

Thus we see that essentially the following three terms have to be computed: $g\left(\mathrm{Y}_{\alpha}, \mathrm{Y}_{\beta}\right), g\left(\mathbf{Y}_{\alpha}, \mathrm{v}\right)$ and $g\left(\mathrm{v}_{1}, \mathrm{v}_{2}\right)$. 
The first term. Since relation 2.2 holds, we get

$$
g\left(\mathbf{Y}_{\alpha}, \mathbf{Y}_{\beta}\right)=g\left(\sum_{r=0}^{3} \alpha_{r} Y_{r}, \sum_{r=0}^{3} \beta_{r} Y_{r}\right)=g\left(Y_{0}, Y_{0}\right) \operatorname{Re}(\alpha \bar{\beta}) .
$$

Recall that $w$ denotes the squared norm of $K_{r}$ and therefore it follows from 2.7 that $g\left(Y_{0}, Y_{0}\right)=w+4 v^{2} v^{-1}=4 v+w$. So finally we have

$$
g\left(\mathbf{Y}_{\alpha}, \mathbf{Y}_{\beta}\right)=(4 v+w) \operatorname{Re}(\alpha \bar{\beta})
$$

The second term. First decompose v into horizontal and vertical parts: $\mathrm{v}=\hat{\mathrm{v}}^{\prime}+\xi(\mathrm{v}) K_{0}$. Taking into account formulae 2.7) again, one obtains

$$
\begin{aligned}
& g\left(\mathrm{Y}_{\beta}, \mathrm{v}\right)=g\left(\mathrm{Y}_{\beta}, \mathrm{v}^{\prime}\right)+2 \beta_{1} \xi(\mathrm{v})= \\
& \quad=\tilde{g}\left(-\beta_{0} I_{1} K_{r}+\beta_{1} K_{r}+\beta_{2} I_{3} K_{r}-\beta_{3} I_{2} K_{r}, \mathrm{v}^{\prime}\right)+2 \beta_{1} \xi(\mathrm{v})= \\
& \quad=\beta_{0} \tilde{\omega}_{1}\left(K_{r}, \mathrm{v}^{\prime}\right)+\beta_{1} \tilde{g}\left(K_{r}, \mathrm{v}^{\prime}\right)-\beta_{2} \tilde{\omega}_{3}\left(K_{r}, \mathrm{v}^{\prime}\right)+\beta_{3} \tilde{\omega}_{2}\left(K_{r}, \mathrm{v}^{\prime}\right)+2 \beta_{1} \xi(\mathrm{v}) .
\end{aligned}
$$

Slightly abusing notations, we also use the letter $\eta$ for the pull-back of the form 2.6 to $P$. Then the above formula can be written in a more compact form:

$$
g\left(\left(R_{m}\right)_{*} \beta, \mathrm{v}\right)=-\operatorname{Re}(2 \beta i \xi(\mathrm{v})+\beta i \eta(\mathrm{v})) .
$$

The third term. This has been already computed and is given by (2.5).

Remark 2.1.2. Below we follow conventions of [35]. In particular, if $\zeta_{1}$ and $\zeta_{2}$ are (quaternion-valued) 1-forms, then

$$
\begin{aligned}
& \left(\zeta_{1} \odot \zeta_{2}\right)\left(v_{1}, v_{2}\right)=\zeta_{1}\left(v_{1}\right) \zeta_{2}\left(v_{2}\right)+\zeta_{1}\left(v_{2}\right) \zeta_{2}\left(v_{1}\right), \\
& \left(\zeta_{1} \wedge \zeta_{2}\right)\left(v_{1}, v_{2}\right)=\zeta_{1}\left(v_{1}\right) \zeta_{2}\left(v_{2}\right)-\zeta_{1}\left(v_{2}\right) \zeta_{2}\left(v_{1}\right) .
\end{aligned}
$$

Now, recalling that $\alpha$ and $\beta$ contain shift by $x^{-1}=\bar{x} /|x|^{2}$ we obtain a final form of the metric:

$$
f^{*} g=(4 v+w) \operatorname{Re} d x \otimes d \bar{x}-\operatorname{Re}(\bar{x} d x i \odot(2 \xi+\eta))+|x|^{2}\left(\tilde{g}+v^{-1} \xi^{2}\right) .
$$

\subsubsection{Symplectic forms}

In this section we will describe symplectic forms in the similar manner as we did with the metric above.

The pull-back of $\omega$ can be written as

$$
\begin{aligned}
f^{*} \omega\left(\left(h_{1}, \mathrm{v}_{1}\right),\left(h_{2}, \mathrm{v}_{2}\right)\right) & =\omega\left(\left(L_{x}\right)_{*}\left(\mathrm{Y}_{\alpha}+\mathrm{v}_{1}\right),\left(L_{x}\right)_{*}\left(\mathrm{Y}_{\beta}+\mathrm{v}_{2}\right)\right)= \\
& =x \omega\left(\mathrm{Y}_{\alpha}+\mathrm{v}_{1}, \mathrm{Y}_{\beta}+\mathrm{v}_{2}\right) \bar{x}
\end{aligned}
$$


where $\alpha$ and $\beta$ are the same as in Section 2.1.2. Therefore we have to compute three terms analogous to those, which appear in the metric computation.

The first term. The computation is similar to the one above:

$$
\begin{aligned}
& \omega\left(\mathbf{Y}_{\alpha}, \mathbf{Y}_{\beta}\right)= \\
& \quad=i g\left(\alpha_{0} Y_{0}+\alpha_{1} Y_{1}+\alpha_{2} Y_{2}+\alpha_{3} Y_{3}, \beta_{0} Y_{1}-\beta_{1} Y_{0}+\beta_{2} Y_{3}-\beta_{3} Y_{2}\right)+ \\
& \quad+j g\left(\alpha_{0} Y_{0}+\alpha_{1} Y_{1}+\alpha_{2} Y_{2}+\alpha_{3} Y_{3}, \beta_{0} Y_{2}-\beta_{1} Y_{3}-\beta_{2} Y_{0}+\beta_{3} Y_{1}\right)+ \\
& \quad+k g\left(\alpha_{0} Y_{0}+\alpha_{1} Y_{1}+\alpha_{2} Y_{2}+\alpha_{3} Y_{3}, \beta_{0} Y_{3}+\beta_{1} Y_{2}-\beta_{2} Y_{1}-\beta_{3} Y_{0}\right)= \\
& \quad=g\left(Y_{0}, Y_{0}\right)\left(i\left(-\alpha_{0} \beta_{1}+\alpha_{1} \beta_{0}-\alpha_{2} \beta_{3}+\alpha_{3} \beta_{2}\right)+\right. \\
& \left.\quad+j\left(-\alpha_{0} \beta_{2}+\alpha_{2} \beta_{0}+\alpha_{1} \beta_{3}-\alpha_{3} \beta_{1}\right)+k\left(-\alpha_{0} \beta_{3}+\alpha_{3} \beta_{0}-\alpha_{1} \beta_{2}+\alpha_{2} \beta_{1}\right)\right)= \\
& \quad=(4 v+w) \operatorname{Im}(\alpha \bar{\beta}) .
\end{aligned}
$$

The second term. Decomposing v into horizontal $\hat{\mathrm{v}}^{\prime}$ and vertical $\xi(\mathrm{v}) K_{0}$ parts one obtains:

$$
\begin{aligned}
& \omega\left(\mathrm{Y}_{\alpha}, \mathrm{v}\right)=i\left(-2 \alpha_{0} \xi(\mathrm{v})+\omega_{1}\left(\left(R_{m}\right)_{*} \alpha, \hat{\mathrm{v}}^{\prime}\right)\right)+ \\
& +j\left(-2 \alpha_{3} \xi(\mathrm{v})+\omega_{2}\left(\left(R_{m}\right)_{*} \alpha, \hat{\mathrm{v}}^{\prime}\right)\right)+k\left(-2 \alpha_{2} \xi(\mathrm{v})+\omega_{3}\left(\left(R_{m}\right)_{*} \alpha, \hat{\mathrm{v}}^{\prime}\right)\right)= \\
& =i\left(-2 \alpha_{0} \xi(\mathrm{v})-\alpha_{0} \tilde{g}\left(K_{r}, \mathrm{v}^{\prime}\right)+\alpha_{1} \tilde{\omega}_{1}\left(K_{r}, \mathrm{v}^{\prime}\right)-\alpha_{2} \tilde{\omega}_{2}\left(K_{r}, \mathrm{v}^{\prime}\right)-\alpha_{3} \tilde{\omega}_{3}\left(K_{r}, \mathrm{v}^{\prime}\right)\right)+ \\
& +j\left(-2 \alpha_{3} \xi(\mathrm{v})+\alpha_{0} \tilde{\omega}_{3}\left(K_{r}, \mathrm{v}^{\prime}\right)+\alpha_{1} \tilde{\omega}_{2}\left(K_{r}, \mathrm{v}^{\prime}\right)+\alpha_{2} \tilde{\omega}_{1}\left(K_{r}, \mathrm{v}^{\prime}\right)-\alpha_{3} \tilde{g}\left(K_{r}, \mathrm{v}^{\prime}\right)\right)+ \\
& +k\left(-2 \alpha_{2} \xi(\mathrm{v})-\alpha_{0} \tilde{\omega}_{2}\left(K_{r}, \mathrm{v}^{\prime}\right)+\alpha_{1} \tilde{\omega}_{3}\left(K_{r}, \mathrm{v}^{\prime}\right)+\alpha_{2} \tilde{g}\left(K_{r}, \mathrm{v}^{\prime}\right)+\alpha_{3} \tilde{\omega}_{1}\left(K_{r}, \mathrm{v}^{\prime}\right)\right)= \\
& =-2 \operatorname{Im}(\alpha i) \xi(\mathrm{v})-\operatorname{Im}(\alpha i \eta(\mathrm{v})) .
\end{aligned}
$$

The third term. It is easy to see that

$$
\omega\left(\mathrm{v}_{1}, \mathrm{v}_{2}\right)=\omega\left(\mathrm{v}_{1}^{\prime}, \mathrm{v}_{2}^{\prime}\right)=\tilde{\omega}\left(\mathrm{v}_{1}, \mathrm{v}_{2}\right),
$$

where the pull-back is also implied.

Thus, recalling that $\alpha=x^{-1} h_{1}=|x|^{-2} \bar{x} h_{1}$, the $\operatorname{Im} \mathbb{H}-$-valued form $\varphi=f^{*} \omega$ can be written as

$$
\varphi=\frac{4 v+w}{2} d x \wedge d \bar{x}+x \tilde{\omega} \bar{x}-2 \operatorname{Im}(d x i \bar{x}) \wedge \xi-\operatorname{Im}(d x i \wedge \eta \bar{x}) .
$$

\subsubsection{Inverse Construction}

Now we can look on the above considerations in reverse order in the following sense. Suppose $\tilde{M}$ is a hyperKähler manifold with metric $\tilde{g}$ and hyperKähler structure $\tilde{\omega}$. Further, a group $S_{r}^{1}$ acts on $\tilde{M}$ preserving complex structure $I_{1}$ and rotating $I_{2}$ and $I_{3}$ in the sense $\left(L_{z}\right)^{*} \tilde{\omega}=z \tilde{\omega} \bar{z}, z \in S_{r}^{1}$. Pick an $S_{0}^{1}$ principal bundle $P$ with a connection $\xi$ and extend the action of $S_{r}^{1}$ to $P$ such that it commutes with $S_{0}^{1}$ (at least locally such extension always exists). 
Consider further a manifold $M_{0}=\mathbb{H}^{*} \times_{S_{r}^{1}} P$. We would like to define a metric $g$ and hyperKähler structure $\omega$ on $M_{0}$ such that their pull-backs to $\mathbb{H}^{*} \times P$ are given by formulae $(2.9)$ and $(2.10)$ respectively. The first thing to show is that these expressions define invariant and basic tensors on $\mathbb{H}^{*} \times P$. One can easily check that both tensors are invariant provided $\xi$ is $S_{r}^{1}$-invariant (see also remark 2.1.5). Let $\chi$ be a Killing vector field of the $S_{r}^{1}$-action on $\mathbb{H}^{*} \times P$. It follows that $\chi=K^{*}-Y_{1}$, where $K^{*}$ is a Killing vector field of the $S_{r}^{1}$-action on $\mathbb{H}^{*}$ by right multiplication, i.e. $d x\left(K^{*}\right)=x i$. Then the equalities $\imath_{\chi} g=0, \imath_{\chi} \varphi=0$ can be checked directly. For example, the last one follows from the following computation:

$$
\begin{aligned}
\left(\imath_{\chi} \varphi\right)(\alpha, \mathrm{v})= & \frac{1}{2}(4 v+w)(x i \bar{\alpha}-\alpha \overline{x i})-x \tilde{\omega}\left(K_{r}, \mathrm{v}\right) \bar{x}- \\
& -2 \operatorname{Im}(x i i \bar{x} \xi(\mathrm{v})+\alpha i \bar{x} 2 v)-\operatorname{Im}\left(x i i \eta(\mathrm{v}) \bar{x}+\alpha i \eta\left(K_{r}\right) \bar{x}\right)= \\
& =(4 v+w) \operatorname{Im}(x i \bar{\alpha})-x \tilde{\omega}\left(K_{r}, \mathrm{v}\right) \bar{x}-2 \cdot 0- \\
& -4 v \operatorname{Im}(x i \bar{\alpha})+x \tilde{\omega}\left(K_{r}, \mathrm{v}\right) \bar{x}-w \operatorname{Im} x i \bar{\alpha}= \\
& =0 .
\end{aligned}
$$

The next question is wether the 2 -form $\omega \in \Omega^{2}\left(M_{0} ; \operatorname{Im} \mathbb{H}\right)$ is closed. As we have seen, the pull-back $\varphi$ of $\omega$ to $\mathbb{H}^{*} \times P$ is basic and therefore this is equivalent to $\varphi$ being closed. Now $d \varphi$ is a quaternion-valued 3-form on $\mathbb{H}^{*} \times P$ and by the Künneth formula $\Omega^{3}\left(\mathbb{H}^{*} \times P ; \operatorname{Im} \mathbb{H}\right) \cong \bigoplus_{l=0}^{3} \Omega^{l}\left(\mathbb{H}^{*} ; \operatorname{Im} \mathbb{H}\right) \otimes$ $\Omega^{3-l}(P ; \operatorname{Im} \mathbb{H})$. Thus $d \varphi$ decomposes in 4 components: $d \varphi=\sum_{l=0}^{3}(d \varphi)_{(l, 3-l)}$, $(d \varphi)_{(l, 3-l)} \in \Omega^{l}\left(\mathbb{H}^{*} ; \operatorname{Im} \mathbb{H}\right) \otimes \Omega^{3-l}(P ; \operatorname{Im} \mathbb{H})$. It is easy to see that $(d \varphi)_{(0,3)}$ and $(d \varphi)_{(3,0)}$ vanish identically and it remains to compute the remaining two components of $d \varphi$.

It follows directly from the expression for $\varphi$ that

$$
\begin{aligned}
(d \varphi)_{(1,2)} & =d x \wedge \tilde{\omega} \bar{x}+x \tilde{\omega} \wedge d \bar{x}+2 \operatorname{Im}(d x i \bar{x}) \wedge F_{\xi}+\operatorname{Im}(d x i \wedge d \eta \bar{x})= \\
& =\operatorname{Im}\left(d x \wedge\left(2 \tilde{\omega}+2 i F_{\xi}+i d \eta\right) \bar{x}\right)
\end{aligned}
$$

and this vanishes iff

$$
-2 i \tilde{\omega}+2 F_{\xi}+d \eta=0 .
$$

By the Cartan formula $[i, \tilde{\omega}]=\mathcal{L}_{K_{r}} \tilde{\omega}=d\left(\imath_{K_{r}} \tilde{\omega}\right)$. But then the above equation can be rewritten as $2 F_{\xi}=-d\left(\imath_{K_{r}} \tilde{g}\right)-d\left(\imath_{K_{r}} \tilde{\omega}\right)+2 i \tilde{\omega}=-d\left(\imath_{K_{r}} \tilde{g}\right)-2 \tilde{\omega}_{1}$. Thus the vanishing of $(d \varphi)_{(1,2)}$ is equivalent to

$$
F_{\xi}=-\frac{1}{2} d\left(\imath_{K_{r}} \tilde{g}\right)-\tilde{\omega}_{1} .
$$


For the other nontrivial component of $d \varphi$ one obtains

$$
\begin{aligned}
(d \varphi)_{(2,1)} & =\frac{1}{2}(4 d v+d w) \wedge d x \wedge d \bar{x}+2 \operatorname{Im}(d x i \wedge d \bar{x}) \wedge \xi-\operatorname{Im}(d x i \wedge \eta \wedge d \bar{x})= \\
& =\frac{1}{2} \operatorname{Im}(d x \wedge(-(4 d v+d w)-4 i \xi-2 i \eta) \wedge d \bar{x})
\end{aligned}
$$

Suppose $\theta$ is a quaternion-valued 1 -form on $\tilde{M}$ and consider the equation $\operatorname{Im}(d x \wedge \theta \wedge d \bar{x})=0$ on $\mathbb{H}^{*} \times \tilde{M}$, which turns out to be equivalent to Re $\theta=0$. Indeed, $d x \wedge \theta \wedge d \bar{x}=-(\operatorname{Re} \theta) \wedge d x \wedge d \bar{x}+d x \wedge \operatorname{Im} \theta \wedge d \bar{x}$ and the last summand is real-valued: $\overline{d x \wedge \operatorname{Im} \theta \wedge d \bar{x}}=(-1) d x \wedge \overline{\operatorname{Im} \theta} \wedge d \bar{x}=d x \wedge \operatorname{Im} \theta \wedge d \bar{x}$.

Therefore $(d \varphi)_{(2,1)}$ vanishes iff

$$
4 d v+d w=2 \imath_{K_{r}} \tilde{\omega}_{1} .
$$

Thus, the 2-form $\varphi$ descends to a closed form on $M_{0}=\mathbb{H}^{*} \times_{S_{r}^{1}} P$ if and only if the three equations are satisfied : (2.8), (2.11) and (2.12). But the last equation follows from the first two. Indeed, since $S_{r}^{1}$ acts isometrically we have $0=\mathcal{L}_{K_{r}}\left(\imath_{K_{r}} \tilde{g}\right)=\imath_{K_{r}} d\left(\imath_{K_{r}} \tilde{g}\right)+d\left(\imath_{K_{r}} \imath_{K_{r}} \tilde{g}\right)$ which means $\imath_{K_{r}} d\left(\imath_{K_{r}} \tilde{g}\right)=-d w$. Now taking the operator $\imath_{K_{r}}$ of both sides of equation (2.11) and using (2.8) we obtain equation 2.12.

It was first remarked in [36] that a hyperKähler manifold with an $S^{1}$ action which preserves one complex structure and permutes the other two has a Kähler potential. Since our conventions slightly differ we reproduce this simple computation.

Let $\tilde{\rho}: \tilde{M} \longrightarrow \mathbb{R}$ be a momentum map of $S_{r}^{1}$, i.e. a solution of the equation

$$
d \tilde{\rho}=-\imath_{K_{r}} \tilde{\omega}_{1} .
$$

On the one hand we have $d\left(I_{2}^{*} d \tilde{\rho}\right)=d i\left(\partial_{2}-\bar{\partial}_{2}\right) \tilde{\rho}=-2 i \partial_{2} \bar{\partial}_{2} \tilde{\rho}$. But on the other hand $I_{2}^{*} \imath_{K_{r}} \tilde{\omega}_{1}=\imath_{K_{r}} \tilde{\omega}_{3}$ and therefore $-d\left(I_{2}^{*} d \tilde{\rho}\right)=d\left(I_{2}^{*} \imath_{K_{r}} \tilde{\omega}_{1}\right)=$ $d\left(\imath_{K_{r}} \tilde{\omega}_{3}\right)=\mathcal{L}_{K_{r}} \tilde{\omega}_{3}=2 \tilde{\omega}_{2}$. Putting this together we obtain that $\tilde{\rho}$ satisfies

$$
i \partial_{2} \bar{\partial}_{2} \tilde{\rho}=\tilde{\omega}_{2}
$$

or, in other words, $\tilde{\rho}$ is a Kähler potential for $\tilde{\omega}_{2}$. It is clear that $\tilde{\rho}$ is also a Kähler potential for $\tilde{\omega}_{3}$ since these forms are not distinguished by the $S_{r}^{1}$ action. However $\tilde{\rho}$ needs not to be a Kähler potential for $\tilde{\omega}_{1}$.

Now if we remark that $I_{1}^{*} d \tilde{\rho}=\imath_{K_{r}} \tilde{g}$ and consequently

$$
-2 i \partial_{1} \bar{\partial}_{1} \tilde{\rho}=d\left(\imath_{K_{r}} \tilde{g}\right),
$$

then equation 2.11) can be written in a particularly nice form: $F_{\xi}=i \partial_{1} \bar{\partial}_{1} \tilde{\rho}-$ $\tilde{\omega}_{1}$, i.e. the function $\tilde{\rho}$ is a hyperKähler potential iff $F_{\xi}=0$. 
Now we can find the function $v$ (up to a constant) from equation 2.12) (or, equivalently, from (2.8)):

$$
v=-\frac{w+2 \tilde{\rho}}{4}
$$

Therefore the following theorem is essentially proven.

Theorem 2.1.3. Let a group $S_{r}^{1}$ act isometrically on a hyperKähler manifold $\tilde{M}$ such that $\left(L_{z}\right)^{*} \tilde{\omega}=z \tilde{\omega} \bar{z}$. Further, let $P \longrightarrow \tilde{M}$ be an $S_{0}^{1}$-principal bundle with a connection $\xi \in \Omega^{1}(P)$. Suppose also, that the function $v$ defined by formula (2.13) is everywhere positive, where $w$ denotes squared norm of the Killing vector field $K_{r}$ of $S_{r}^{1}$, while $\tilde{\rho}$ is its momentum map. Extend the action of $S_{r}^{1}$ to $P$ such that it commutes with the action of $S_{0}^{1}$. Then (2.9) and (2.10) define a hyperKähler structure on $M_{0}=\mathcal{H}(\tilde{M})=\mathbb{H}^{*} \times_{S_{r}^{1}} P$ if and only if

$$
F_{\xi}=i \partial_{1} \bar{\partial}_{1} \tilde{\rho}-\tilde{\omega}_{1}
$$

Furthermore the left action of $\mathbb{H}^{*}$ induces a transitive action on the 2sphere of complex structures and therefore $\mathcal{H}(\tilde{M})$ has a hyperKähler potential

$$
\rho=-\frac{4 v+w}{2}|x|^{2}
$$

Finally, for any hyperKähler manifold $M$ with permuting action of $\mathbb{H}^{*}$ and triholomorphic one of $S^{1}$, the open everywhere dense submanifold $M_{0}=$ $M \backslash \mu^{-1}(0)$ can be obtained as $\mathcal{H}(\tilde{M})$, where $\tilde{M}$ is as above.

Proof. It remains to show that the symmetric tensor given by formula (2.9) provides a non-negative bilinear form at any point of the tangent space to $\mathbb{H}^{*} \times P$ as well as to prove formula 2.15 for the hyperKähler potential.

First we have a decomposition $T\left(\mathbb{H}^{*} \times P\right)=T \mathbb{H} \oplus T P=T \mathbb{H} \oplus \mathbb{R} K_{0} \oplus$ $\pi^{*} T \tilde{M}$. Further decompose $T \tilde{M}$ as $\operatorname{span}\left(K_{r}, I_{1} K_{r}, I_{2} K_{r}, I_{3} K_{r}\right) \oplus E$, where $E$ denotes the orthogonal complement. Thus we have

$$
T\left(\mathbb{H}^{*} \times P\right)=T \mathbb{H} \oplus \mathbb{R} K_{0} \oplus \pi^{*} \operatorname{span}\left(K_{r}, I_{1} K_{r}, I_{2} K_{r}, I_{3} K_{r}\right) \oplus \pi^{*} E,
$$

and we can write a tangent vector as $\mathrm{w}=\mathrm{w}^{*}+a K_{0}+\beta K_{r}+\mathrm{v}$, where $a$ is a 
real number and $\beta$ is a quaternion ${ }^{b}$. If $d x\left(\mathrm{w}^{*}\right)=\alpha \in \mathbb{H}$, then

$$
\begin{aligned}
g(\mathrm{w}, \mathrm{w}) & =(4 v+w)|\alpha|^{2}+|x|^{2}\left(|\beta|^{2}+\|\mathrm{v}\|^{2}+\frac{a^{2}}{v}\right)-2 \operatorname{Re}(\bar{x} \alpha i(2 a+w \beta))= \\
& =4 v|\alpha|^{2}-4 a \operatorname{Re}(\bar{x} \alpha i)+|x|^{2} v^{-1} a^{2}+ \\
& +w\left(|\alpha|^{2}-2 \operatorname{Re}(\bar{x} \alpha i \beta)+|\beta x|^{2}\right)+|x|^{2}\|\mathrm{v}\|^{2}= \\
& =\left|2 \sqrt{v} \alpha i-\frac{a x}{\sqrt{v}}\right|^{2}+w|\alpha i-\beta \bar{x}|^{2}+|x|^{2}\|\mathrm{v}\|^{2} \geq 0 .
\end{aligned}
$$

Further, it was shown in [11 that if a hyperKähler manifold $M$ admits a permuting $\mathbb{H}^{*}$-action, then the squared norm of any Killing vector field generating this action is a hyperKähler potential (up to a constant $-2^{\text {C }}$ ). Now the permuting action of $\mathbb{H}^{*}$ on $\mathcal{H}(\tilde{M})$ is induced by the left multiplication on the first component of $\mathbb{H}^{*} \times P$. In particular, the Killing vector field of $\mathbb{R}^{*} \subset$ $\mathbb{H}^{*}$ is the vector field $\mathrm{w}^{*}$ s.t. $d x\left(\mathrm{w}^{*}\right)=x$. Its squared norm (multiplied by $-1 / 2)$ with respect to metric $(2.9)$ is exactly the right-hand side of (2.15).

Remark 2.1.4. It is easy to see that the hyperKähler reduction of $\mathcal{H}(\tilde{M})$ by $S_{0}^{1}$ is $\tilde{M}$ (certainly not a surprise in view of Section 2.1.1). Thus the construction $\mathcal{H}(\cdot)$ may be regarded as a kind of "hyperKähler induction", i.e. an inverse construction to the hyperKähler reduction.

Remark 2.1.5. The equation (2.14) determines the bundle $P$ : its Chern class is $-\frac{1}{2 \pi}\left[\tilde{\omega}_{1}\right] \in H^{2}(\tilde{M} ; \mathbb{Z})$ and the integrality of $\frac{1}{2 \pi}\left[\tilde{\omega}_{1}\right]$ is the only obstruction for the existence of solutions to (2.14). Furthermore, any solution $\xi$ is automatically $S_{r}^{1}$-invariant and the Killing vector field $Y_{1}$ of $S_{r}^{1}$-action on $P$ satisfies $Y_{1}=\hat{K}_{r}+2 v K_{0}$. Indeed, as we have seen the right hand side of equation (2.14) may be written in the form $-\frac{1}{2} d\left(\imath_{K_{r}} \tilde{g}\right)-\tilde{\omega}_{1}$ and this immediately implies $S_{r}^{1}$-invariancy of $\xi$. Further, we may decompose $Y_{1}$ on the horizontal and vertical parts: $Y_{1}=\hat{K}_{r}+a K_{0}$. Then by Remark 2.1.1 we have $\imath_{K_{r}} F_{\xi}+d a=0$. On the other hand equation (2.14) implies $\imath_{K_{r}} F_{\xi}=-2 d v$ and the statement follows.

It is worth pointing out that equality $a=2 v$ holds only up to a constant. This phenomenon will be discussed in details in Section 2.3 below. At this point we will ignore this subtlety implying that a constant is chosen properly, i.e. such that equation $Y_{1}=\hat{K}_{r}+2 v K_{0}$ holds.

\footnotetext{
${ }^{\mathrm{b}}$ Any tangent space of a hyperKähler manifold carries an action of $\mathbb{H}$. In particular if $\beta=\beta_{0}+\beta_{1} i+\beta_{2} j+\beta_{3} k \in \mathbb{H}$, we write $\beta K_{r}$ instead of $\beta_{0} K_{r}+\sum_{l=1}^{3} \beta_{l} I_{l} K_{r}$ for the sake of brevity.

${ }^{c}$ the minus sign appears because of different sign convention in the definition of hyperKähler potential.
} 
Remark 2.1.6 (Complex structures). We would like to indicate how one can describe complex structures of $\mathcal{H}(\tilde{M})$. It is convenient for a while to index complex structures of a hyperKähler manifold by imaginary quaternions $q$ of unit length. Let $I_{q}$ be one of the complex structures of $M$ and $(h, \mathrm{v}) \in$ $T \mathbb{H}^{*} \times T P$. Consider the equation

$$
f_{*}\left(h_{1}, \mathrm{v}_{1}\right)=I_{q} f_{*}(h, \mathrm{v}),
$$

where the map $f$ is as defined in (2.4). Its solution $\left(h_{1}, \mathrm{v}_{1}\right)=J_{q}(h, \mathrm{v})$ is defined only up to the Killing vector field $\chi$; although the operator $J_{q}$ is not defined on $T\left(\mathbb{H}^{*} \times P\right)$, it is well-defined on the factorspace $T\left(\mathbb{H}^{*} \times P\right) / \operatorname{span}(\chi)$. Since we are free to add any vector of $\operatorname{span}(\chi)$ to both $(h, \mathrm{v})$ and $\left(h_{1}, \mathrm{v}_{1}\right)$ it can be assumed that $\xi(\mathrm{v})=0=\xi\left(\mathrm{v}_{1}\right)$. Under these conventions equation (2.16) has a unique solution $J_{q}(h, \mathrm{v})=\left(q h, I_{x^{-1} q x} \mathrm{v}\right)$.

Remark 2.1.7. Suppose that the $S_{r}^{1}$-action is induced by a permuting $\mathbb{H}^{*}$ action (and standard inclusion $S_{r}^{1} \subset \mathbb{H}^{*}$ ), or equivalently, the momentum map of the $S_{r}^{1}$-action is not only Kähler potential but also hyperKähler [65]. It follows from equation (2.14) that the bundle $P$ is flat and we can take it to be trivial so that topologically $\mathcal{H}(\tilde{M})=\mathbb{H}^{*} \times \tilde{M}$. Moreover, it follows from the proof of the theorem that $v$ is constant so that we may put $v=1$. This determines a metric and symplectic forms.

Further, it turns out that in this case $\mathcal{H}(\tilde{M})$ is isometric to $\mathbb{H}^{*} \times \tilde{M}$ with its product metric. Indeed, direct computation shows that the map $\mathcal{H}(\tilde{M}) \longrightarrow \mathbb{H}^{*} \times \tilde{M},(x, m) \mapsto(x, x m)$ is an isometry.

\subsubsection{Quaternionic Flip}

In the previous section for any hyperKähler manifold $\tilde{M}$ with a certain $S^{1}$ symmetry we have constructed another hyperKähler manifold $M_{0}=\mathcal{H}(\tilde{M})$ with hyperKähler potential. Then Swann's results [65] imply that the manifold $N_{0}=M_{0} / \mathbb{H}^{*}=P / S_{r}^{1}$ is quaternionic Kähler. In this section we will describe its quaternionic Kähler structure.

First notice that in order to obtain quaternionic Kähler structure on $N_{0}$ we have to consider a riemannian version of the quotient $M_{0} / \mathbb{H}^{*}$, that is to pick a level set of a hyperKähler potential and divide it by the group $S p(1)$; in this case we may view complex structures of $N_{0}$ as induced by those of $M_{0}$ on $\operatorname{span}\left(Y_{0}, Y_{1}, Y_{2}, Y_{3}\right)^{\perp} \subset T M_{0}$.

Let us again return to the viewpoint of Section 2.1.1, i.e. $P=\mu^{-1}(i) \subset M$ and let $\lambda=(4 v+w)^{-1 / 2}$. Since the restriction of the hyperKähler potential $\rho$ to $P$ equals $-(4 v+w) / 2$, a map

$$
l: p \mapsto \lambda(p) \cdot p=L_{\lambda(p)} p, \quad p \in P,
$$


is a diffeomorphism between $P$ and $Q=\rho^{-1}(-1 / 2) \cap \mu_{c}^{-1}(0) \cap\left\{\mu_{1}>0\right\}$, where $\mu_{c}=\mu_{2}+i \mu_{3}$. Thus our next aim is to compute the tensors $g\left(\operatorname{pr} \circ l_{*} \cdot, \operatorname{pr} \circ l_{*} \cdot\right)$ and $\omega\left(\operatorname{prol}_{*} \cdot, \operatorname{prol}_{*} \cdot\right)$, where $p r$ means a projection onto $\operatorname{span}\left(Y_{0}, Y_{1}, Y_{2}, Y_{3}\right)^{\perp}$.

First we may decompose a vector $\mathrm{u} \in T_{p} P \subset T_{p} M$ as $\mathrm{u}^{\prime}+\sum_{l=0}^{3} a_{l} Y_{l}$. The coefficients $a_{l}$ can be found from the following relations:

$$
\begin{aligned}
& a_{0} g\left(Y_{0}, Y_{0}\right)=g\left(\mathrm{u}, Y_{0}\right)=g\left(\mathrm{u},-I_{1} \hat{K}_{r}-2 v I_{1} K_{0}\right)=\tilde{\omega}_{1}\left(K_{r}, \mathrm{u}\right), \\
& a_{1} g\left(Y_{1}, Y_{1}\right)=g\left(\mathrm{u}, Y_{1}\right)=g\left(\mathrm{u}, \hat{K}_{r}+2 v K_{0}\right)=2 \xi(\mathrm{u})+\tilde{g}\left(K_{r}, \mathrm{u}\right), \\
& a_{2} g\left(Y_{2}, Y_{2}\right)=g\left(\mathrm{u}, Y_{2}\right)=g\left(\mathrm{u}, I_{3} \hat{K}_{r}+2 v I_{3} K_{0}\right)=-\tilde{\omega}_{3}\left(K_{r}, u\right), \\
& a_{3} g\left(Y_{3}, Y_{3}\right)=g\left(\mathrm{u}, Y_{3}\right)=g\left(\mathrm{u},-I_{2} \hat{K}_{r}-2 v I_{2} K_{0}\right)=\tilde{\omega}_{2}\left(K_{r}, u\right) .
\end{aligned}
$$

The expressions for $a_{l}$ become more compact in quaternionic notations. Indeed, if we put $a=a_{0}+a_{1} i+a_{2} j+a_{3} k \in \mathbb{H}$ and recall the definition (2.6) of 1-form $\eta$, then

$$
a=\frac{1}{4 v+w}(2 \xi(\mathrm{u})+\bar{\eta}(\mathrm{u})) i .
$$

Since $l_{*}=\left(L_{\lambda(p)}\right)_{*}+d \lambda Y_{0}(l(p))$, we have $\operatorname{pr} l_{*} \mathrm{~V}=\left(L_{\lambda(p)}\right)_{*} \mathrm{v}^{\prime}$ and therefore

$$
\begin{aligned}
g\left(\operatorname{pr} l_{*} \mathrm{u}, \operatorname{pr} l_{*} \mathrm{v}\right) & =g\left(\left(L_{\lambda(p)}\right)_{*} \mathrm{u}^{\prime},\left(L_{\lambda(p)}\right)_{*} \mathrm{v}^{\prime}\right)= \\
& =\lambda^{2} g\left(\mathrm{u}^{\prime}, \mathrm{v}^{\prime}\right)= \\
& =\lambda^{2} g\left(\mathrm{u}-a Y_{0}, \mathrm{v}-b Y_{0}\right)= \\
& =\lambda^{2}\left(g(\mathrm{u}, \mathrm{v})-g\left(a Y_{0}, \mathrm{v}\right)-g\left(\mathrm{u}, b Y_{0}\right)+g\left(a Y_{0}, b Y_{0}\right)\right),
\end{aligned}
$$

where we also put $\mathrm{v}=\mathrm{v}^{\prime}+b Y_{0}$. Now it is easy to compute every single summand in the last expression. Indeed, the first summand is given by formula (2.5). Taking into account decompositions (2.7) one obtains $g\left(a Y_{0}, \mathrm{v}\right)=$ $-\operatorname{Re}(\operatorname{ai}(2 \xi+\eta)(\mathrm{v}))=(4 v+w)^{-1} \operatorname{Re}((2 \xi+\bar{\eta})(\mathrm{u})(2 \xi+\eta)(\mathrm{v}))$. Since vectors $Y_{l}$ are pairwise orthogonal we get: $g\left(a Y_{0}, b Y_{0}\right)=(4 v+w) \operatorname{Re}(a \bar{b})=$ $(4 v+w)^{-1} \operatorname{Re}((2 \xi+\bar{\eta})(\mathrm{u})(2 \xi+\eta)(\mathrm{v}))$. Finally, gathering all terms together one has after a simplification:

$$
g_{\mathrm{N}}=\frac{1}{4 v+w}\left(\tilde{g}+\frac{1}{v} \xi^{2}-\frac{1}{2(4 v+w)}(2 \xi+\bar{\eta}) \odot(2 \xi+\eta)\right) .
$$

It is convenient to introduce a 1 -form

$$
\psi=\frac{1}{g\left(Y_{1}, Y_{1}\right)} g\left(Y_{1}, \cdot\right)=\frac{1}{4 v+w}\left(2 \xi+\imath_{K_{r}} \tilde{g}\right),
$$

which is a connection on the principal fibre bundle $P \longrightarrow N_{0}$ (assuming that $S_{r}^{1}$ acts freely on $P$ ), i.e. it is $S_{r}^{1}$-invariant and $\psi\left(Y_{1}\right)=1$. Then the 
expression for the metric takes the following form:

$$
g_{\mathrm{N}}=\frac{1}{4 v+w}\left(\tilde{g}+\frac{1}{v} \xi^{2}-\frac{1}{2} \psi^{2}\right)-\frac{1}{2(4 v+w)^{2}} \sum_{l=1}^{3}\left(\imath_{K_{r}} \tilde{\omega}_{l}\right)^{2} .
$$

The arguments similar to those at the beginning of Section 2.1.4 show that formula (2.19) defines a metric on $N_{0}$.

The fundamental 4-form $\Omega$ can be obtained in the similar manner. Indeed,

$$
\begin{aligned}
\chi(\mathrm{u}, \mathrm{v}) & =\omega\left(\operatorname{pr} l_{*} \mathrm{u}, \operatorname{pr} l_{*} \mathrm{v}\right)=\lambda^{2} \omega\left(\mathrm{u}-a Y_{0}, \mathrm{v}-b Y_{0}\right)= \\
& =\lambda^{2}\left(\omega(\mathrm{u}, \mathrm{v})-\omega\left(a Y_{0}, \mathrm{v}\right)-\omega\left(\mathrm{u}, b Y_{0}\right)+\omega\left(a Y_{0}, b Y_{0}\right)\right) .
\end{aligned}
$$

Arguing similarly as we did when computing the metric, we obtain finally

$$
\chi=\frac{1}{4 v+w} \tilde{\omega}-\frac{1}{2(4 v+w)^{2}}(2 \xi+\bar{\eta}) \wedge(2 \xi+\eta) .
$$

Componentwise spelling of this formula is

$$
\begin{aligned}
& \chi_{1}=\frac{1}{4 v+w}\left(\tilde{\omega}_{1}-\psi \wedge \imath_{K_{r}} \tilde{\omega}_{1}\right)+\frac{1}{(4 v+w)^{2}} \imath_{K_{r}} \tilde{\omega}_{2} \wedge \imath_{K_{r}} \tilde{\omega}_{3}, \\
& \chi_{2}=\frac{1}{4 v+w}\left(\tilde{\omega}_{2}-\psi \wedge \imath_{K_{r}} \tilde{\omega}_{2}\right)-\frac{1}{(4 v+w)^{2}} \imath_{K_{r}} \tilde{\omega}_{1} \wedge \imath_{K_{r}} \tilde{\omega}_{3}, \\
& \chi_{3}=\frac{1}{4 v+w}\left(\tilde{\omega}_{3}-\psi \wedge \imath_{K_{r}} \tilde{\omega}_{3}\right)+\frac{1}{(4 v+w)^{2}} \imath_{K_{r}} \tilde{\omega}_{1} \wedge \imath_{K_{r}} \tilde{\omega}_{2} .
\end{aligned}
$$

With respect to the action of $S_{r}^{1}$ on $P$ all three forms $\chi_{l} \in \Omega^{2}(P)$ are basic, however only $\chi_{1}$ is invariant:

$$
\begin{aligned}
\mathcal{L}_{Y_{1}} \chi_{1} & =\frac{1}{(4 v+w)^{2}}\left(-2 \imath_{K_{r}} \tilde{\omega}_{3} \wedge \imath_{K_{r}} \tilde{\omega}_{3}+\imath_{K_{r}} \tilde{\omega}_{2} \wedge 2 \imath_{K_{r}} \tilde{\omega}_{2}\right)=0, \\
\mathcal{L}_{Y_{1}} \chi_{2} & =\frac{1}{4 v+w}\left(-2 \tilde{\omega}_{3}+\psi \wedge 2 \imath_{K_{r}} \tilde{\omega}_{3}\right)+\frac{1}{(4 v+w)^{2}} \imath_{K_{r}} \tilde{\omega}_{1} \wedge 2 \imath_{K_{r}} \tilde{\omega}_{2}=-2 \chi_{3}, \\
\mathcal{L}_{Y_{1}} \chi_{3} & =\frac{1}{4 v+w}\left(2 \tilde{\omega}_{2}-\psi \wedge 2 \imath_{K_{r}} \tilde{\omega}_{2}\right)-\frac{1}{(4 v+w)^{2}} \imath_{K_{r}} \tilde{\omega}_{1} \wedge 2 \imath_{K_{r}} \tilde{\omega}_{3}=2 \chi_{2},
\end{aligned}
$$

It follows that a $4-$ form

$$
\Omega=\chi_{1} \wedge \chi_{1}+\chi_{2} \wedge \chi_{2}+\chi_{3} \wedge \chi_{3}
$$

is basic and invariant and therefore descends to $N_{0}$. Integrability of such defined quaternionic Kähler structure follows from integrability of the hyperKähler structure on $M_{0}=\mathcal{H}(\tilde{M})=\mathcal{U}\left(N_{0}\right)$ [65].

Theorem 2.1.8. Let the assumptions of Theorem 2.1.3 be satisfied. Then $N_{0}=\mathcal{Q}(\tilde{M})=P / S_{r}^{1}$ is a quaternionic Kähler manifold with respect to the metric (2.19) and the fundamental 4 -form (2.21), where $\chi_{l}$ and $\psi$ are defined by (2.20) and (2.18) respectively. Moreover $\mathcal{Q}(\tilde{M})$ admits a quaternionic Kähler action of $S^{1}$ and its Swann bundle $\mathcal{U}\left(N_{0}\right)$ is $\mathcal{H}(\tilde{M})$. 


\section{$2.2 \quad$ Examples}

Example 2.2.1 ( $T^{*} \mathbb{C P}^{n}$ with the Calabi metric). The hyperKähler quotient of $\mathbb{H}^{n+1}$ by $S^{1}$ acting by multiplication on the left with respect to nonzero value of momentum map is topologically $T^{*} \mathbb{C P}^{n}$. Hitchin [37] shows, that the metric coincides with the one defined by Calabi 13. Therefore $\mathcal{H}\left(T^{*} \mathbb{C P}^{n}\right)=\mathbb{H}^{n+1}$ with its flat metric and $\mathcal{Q}\left(T^{*} \mathbb{C P}^{n}\right)=\mathbb{H} \mathbb{P}^{n}$ (in both cases with zero level set of corresponding momentum map being removed).

Example 2.2.2 (Flat manifold, adjoint action). Let us take a copy of quaternions $\mathbb{H}_{y}$ as a manifold $\tilde{M}$ with the following action of $S_{r}^{1}:(z, y) \mapsto z y \bar{z}$ (one can also regard $\mathbb{H}$ as $T^{*} \mathbb{C}$ with fibrewise action of $S_{r}^{1}$; see also remark 2.2.3). In this case $1 / 4(w+2 \tilde{\rho})=1 / 2\left(y_{2}^{2}+y_{3}^{2}\right)$, where $y=y_{0}+y_{1} i+y_{2} j+y_{3} k$. Adding $1 / 2$ we may write function $v$ in the form

$$
v=\frac{1}{2}\left(1-y_{2}^{2}-y_{3}^{2}\right)
$$

and it is positive on $\mathbb{R}_{y_{0} y_{1}}^{2} \times \mathrm{D}_{y_{2} y_{3}}^{2}$, where $\mathrm{D}^{2} \subset \mathbb{R}^{2}$ is an open disc of radius 1 . The principal bundle $P$ is trivial and therefore $\mathcal{Q}\left(\mathbb{R}^{2} \times \mathrm{D}^{2}\right)=\mathbb{R}^{2} \times \mathrm{D}^{2}$ with the following metric:

$$
\begin{array}{r}
g_{\mathrm{N}}=\frac{1}{2(1+d)}\left(\frac{1-d}{1+d} \operatorname{Re} d y \otimes d \bar{y}+\frac{4 d}{1-d^{2}}\left(y_{0} d y_{1}+y_{3} d y_{2}\right)^{2}-\right. \\
\left.-\frac{1}{1+d}\left(y_{0} d y_{1}+y_{3} d y_{2}\right) \odot\left(y_{2} d y_{3}-y_{3} d y_{2}\right)\right),
\end{array}
$$

where $d=y_{2}^{2}+y_{3}^{2}$. Therefore the above metric is Einstein and self-dual. However it is incomplete.

Similarly, one can compute the metric and symplectic forms on $\mathcal{H}\left(\mathbb{R}^{2} \times\right.$ $\left.\mathrm{D}^{2}\right)=\mathbb{H}^{*} \times \mathbb{R}^{2} \times \mathrm{D}^{2}$ but the metric is also incomplete.

Remark 2.2.3. The above manifolds are examples of a large class of hyperKähler manifolds admitting $S_{r}^{1}$-action. Namely Kaledin [43] and independently Feix [24] proved the existence of a hyperKähler metric on a (neighborhood of the zero section of) cotangent bundle $T^{*} \mathcal{M}$ to a real-analytic Kähler manifold $\mathcal{M}$. The above examples show that the function $v$ can be both positive everywhere and only on a proper open subset of $T^{*} \mathcal{M}$; also $\mathcal{H}\left(T^{*} \mathcal{M}\right)$ can be both complete and incomplete.

Example 2.2.4 (Gibbons-Hawking Spaces). As we have already mentioned in the introduction, all hyperKähler 4-manifolds with $S^{1}$-symmetry were described by Gibbons and Hawking 34 and their construction is as 
follows. If $Z^{4}$ is hyperKähler and admits $S^{1}$-symmetry with a Killing vector field $K_{0}$, then its hyperKähler momentum map $\mu=\mu_{1} i+\mu_{2} j+\mu_{3} k$ represents $Z$ as a fibration over $\mathbb{R}^{3}$ with generic fibre $S^{1}$, so that, excluding critical points of the momentum map, one can write the metric as

$$
g_{\mathrm{GH}}=\nu\left(d x_{1}^{2}+d x_{2}^{2}+d x_{3}^{2}\right)+\nu^{-1} \xi^{2}, \quad x_{l}=\mu_{l}, l=1,2,3,
$$

where $\nu: \mathbb{R}^{3} \longrightarrow \mathbb{R}_{>0}, \nu^{-1}=\left\|K_{0}\right\|^{2}$, and $\xi$ is a connection form. It is then an easy exercise to write down 2 -forms which are closed provided

$$
F_{\xi}=-* d \nu
$$

It follows form the Bianchi identity that $\nu$ is harmonic. We would like to point out that $Z^{4}$ is determined by the function $\nu$ (harmonic and positive) since the connection $\xi$ can be found from equation (2.23). The GibbonsHawking-Ansatz is a choice of a particular function $\nu$ :

$$
\nu(x)=\sum_{i=1}^{n} \frac{1}{\left|x-y_{i}\right|}, \quad y_{i} \in \mathbb{R}^{3} .
$$

In general the above 4-manifold does not admit an $S_{r}^{1}$-action. However when all the poles $y_{i}$ of the function $\nu$ lie on one line (say $x_{1}$-axis), then such an action does exist; its projection to $\mathbb{R}^{3} \cong \operatorname{Im} \mathbb{H}$ is then $(z, x) \mapsto$ $z x \bar{z}, x \in \operatorname{Im} \mathbb{H}$. A direct (and tedious) computation shows that the function $v$ (see $(2.13)$ ) is everywhere positive and therefore the construction $\mathcal{H}(Z)$ (see Theorem 2.1.3 is defined on the whole Gibbons-Hawking space $Z$. However we will construct it in a different way. by

Let a torus $\mathbb{T}^{n}=S_{z_{1}}^{1} \times \cdots \times S_{z_{n}}^{1}$ act on a flat hyperKähler manifold $\mathbb{H}^{n+1}$

$$
\left(z_{1}, \ldots, z_{n}, y_{1}, \ldots, y_{n+1}\right) \mapsto\left(y_{1} z_{1} \bar{z}_{2} \ldots \bar{z}_{n}, y_{2} z_{1} z_{2}, \ldots, y_{n} z_{1} z_{n}, y_{n+1} z_{1}\right)
$$

Consider a hyperKähler reduction $Z_{n}$, i.e. the space of solutions to

$$
\begin{aligned}
& y_{1} i \bar{y}_{1}+y_{2} i \bar{y}_{2}+y_{3} i \bar{y}_{3} \cdots+y_{n} i \bar{y}_{n}+y_{n+1} i \bar{y}_{n+1}=c_{1} i, \\
& -y_{1} i \bar{y}_{1}+y_{2} i \bar{y}_{2}=c_{2} i, \\
& -y_{1} i \bar{y}_{1}+y_{3} i \bar{y}_{3}=c_{3} i, \\
& -y_{1} i \bar{y}_{1}+y_{n} i \bar{y}_{n} \quad=c_{n} i,
\end{aligned}
$$

modulo $\mathbb{T}^{n}$, where $c_{l} \in \mathbb{R}$. It is an example of a 4 -dimensional toric hyperKähler manifold [10]. The following action of $S^{1}$

$$
\left(w, y_{1}, \ldots, y_{n+1}\right) \mapsto\left(y_{1}, \ldots, y_{n}, y_{n+1} w\right)
$$


descends to a hyperKähler one on $Z_{n}$. It follows that $Z_{n}$ is a GibbonsHawking space. In order to identify it we have to compute the function $\nu$ (see $(2.22)$ ). The Killing vector field of the action 2.25) is $K_{0}=\left(0, \ldots, 0, y_{n+1} i\right)$ and $\nu^{-1}$ is a squared norm of $K_{0}$ with respect to the metric of hyperKähler reduction, i.e. squared norm of the projection of $K_{0}$ to $V^{\perp}$, where $V$ is spanned by the Killing vector fields of $\mathbb{T}^{n}: V=\operatorname{span}\left(K_{1}, \ldots, K_{n}\right)$ and

$$
\begin{aligned}
& K_{1}=\left(y_{1} i, y_{2} i, y_{3} i, \ldots, y_{n} i, y_{n+1} i\right) \text {, } \\
& K_{2}=\left(-y_{1} i, y_{2} i, 0, \ldots, 0,0\right) \text {, } \\
& K_{3}=\left(-y_{1} i, 0, y_{3} i, \ldots, 0,0\right) \text {, } \\
& K_{n}=\left(-y_{1} i, 0,0, \ldots, y_{n} i, 0\right) \text {. }
\end{aligned}
$$

If we write the projection of $K_{0}$ on $V$ as $u_{1} K_{1}+\cdots+u_{n} K_{n}$, then the coefficients $u_{1}, \ldots, u_{n}$ are found from the system $\sum_{s=1}^{n}\left\langle K_{l}, K_{s}\right\rangle u_{s}=\left\langle K_{l}, K_{0}\right\rangle, l=\overline{1, n}$. Denote $a_{s}=\left|y_{s}\right|^{2}$ for a while; then the above mentioned system has the following form

$$
\begin{aligned}
& \sum_{s=0}^{n} a_{s} u_{1}+\left(a_{2}-a_{1}\right) u_{2}+\left(a_{3}-a_{1}\right) u_{3}+\cdots+\left(a_{n}-a_{1}\right) u_{n}=a_{n+1}, \\
& \left(a_{2}-a_{1}\right) u_{1}+\left(a_{2}+a_{1}\right) u_{2}+\quad a_{1} u_{3}+\cdots+\quad a_{1} u_{n}=0 \text {, } \\
& \left(a_{3}-a_{1}\right) u_{1}+\quad a_{1} u_{2}+\left(a_{3}+a_{1}\right) u_{3}+\cdots+\quad a_{1} u_{n}=0, \\
& \left(a_{n}-a_{1}\right) u_{1}+\quad a_{1} u_{2}+\quad a_{1} u_{3}+\cdots+\left(a_{1}+a_{n}\right) u_{n}=0 .
\end{aligned}
$$

A direct computation shows that

$$
\begin{aligned}
& u_{1}=a_{n+1} \sum_{i=1}^{n} a_{1} \ldots \hat{a}_{i} \ldots a_{n} / \Delta \\
& u_{s}=a_{n+1}\left((n+1) a_{1} \ldots \hat{a}_{s} \ldots a_{n}-\sum_{i=1}^{n} a_{1} \ldots \hat{a}_{i} \ldots a_{n}\right) / \Delta, \quad s=\overline{2, n}
\end{aligned}
$$

is a solution of system (2.26), where $\Delta=n^{2} a_{1} \cdots a_{n}+\sum a_{1} \ldots \hat{a}_{i} \ldots a_{n+1}$ and $\hat{a}_{i}$ means that this factor is omitted in the corresponding monomial.

Further,

$$
\nu^{-1}=\left\|p r_{V^{\perp}} K_{0}\right\|^{2}=\left\|K_{0}\right\|^{2}-\left\|\sum_{s} u_{s} K_{s}\right\|^{2}=a_{n+1}-\sum_{l, s}\left\langle K_{l}, K_{s}\right\rangle u_{l} u_{s}
$$

Now multiply the $l$ 's equation of system 2.26 by $u_{l}$ and sum up. Recall also that the coefficients of system (2.26) are exactly $\left\langle K_{l}, K_{s}\right\rangle$ and therefore 
$\nu^{-1}=a_{n+1}-a_{n+1} u_{1}$. After a simple transformation one obtains

$$
\nu=\frac{1}{a_{n+1}}+\frac{1}{n^{2}} \sum_{s=1}^{n} \frac{1}{a_{s}}=\frac{1}{\left|y_{n+1}\right|^{2}}+\frac{1}{n^{2}} \sum_{s=1}^{n} \frac{1}{\left|y_{s}\right|^{2}} .
$$

Now we have to express $\nu$ as a function of $x=y_{n+1} i \bar{y}_{n+1} \in \operatorname{Im} \mathbb{H} \cong \mathbb{R}^{3}$ since $y_{n+1} i \bar{y}_{n+1}$ is the momentum map of action 2.25).

First, one has $\left|y_{n+1}\right|^{2}=|x|$ for free. Then it is easy to find each term $y_{l} i \bar{y}_{l}$ from the system 2.24). Indeed, subtracting from the first equation the sum of the rest, one gets $n y_{1} i \bar{y}_{1}=-x+\left(c_{1}-c_{2}-\cdots-c_{n}\right) i$; now one easily has $n y_{l} i \bar{y}_{l}=-x+d_{l} i, d_{l}=c_{1}+(n-1) c_{l}-\sum_{s \neq l, s \geq 2} c_{s}$. Remark that one can choose constants $c_{l}$ such that all $d_{l}$ 's are different. Thus $\left|y_{l}\right|^{2}=\left|x-d_{l} i\right|$ and therefore we have

$$
\nu=\frac{1}{|x|}+\frac{1}{n} \sum_{l=1}^{n} \frac{1}{\left|x-d_{l} i\right|} .
$$

Proposition 2.2.5. The Gibbons-Hawking space $Z_{n}$ corresponding to the function $\nu$ with $n+1$ poles (2.27) is a hyperKähler reduction of the flat space $\mathbb{H}^{n+1}$ by a torus $\mathbb{T}^{n}$. Similarly, the manifold $\mathcal{H}\left(Z_{n}\right)$ is a hyperKähler reduction of $\mathbb{H}^{n+1}$ by $\mathbb{T}^{n-1}$, i.e. both are toric hyperKähler manifolds.

Remark 2.2.6. The fact, that Gibbons-Hawking spaces can be obtained as hyperKähler reductions of a flat space by a torus has long been known in physical literature: see [31] and references therein.

Proof. It remains to prove that $\mathcal{H}\left(Z_{n}\right)$ is a toric hyperKähler manifold. First observe that $Z_{1}$ is a hyperKähler reduction of $\mathbb{H}^{2}$ by a circle and therefore is homothetic to $T^{*} \mathbb{C P}^{1}$ with Calabi metric when $c_{1} \neq 0$ (see (2.24)) or $\mathbb{H}$ with its flat metric when $c_{1}=0$. In both cases $\mathcal{H}\left(Z_{1}\right)=\mathbb{H}^{2}$.

If $n=2$ we may choose constants $\left(c_{1}, c_{2}\right)$ to be $(0,1)$. It is well known (see [65], for example) that the hyperKähler reduction $\mathbb{H}^{3} / / / S_{z_{1}}^{1}$ with respect to the zero value of the momentum map is a nilpotent orbit $\mathcal{O}$; consequently $\mathcal{O}$ has a hyperKähler potential. Further the space $Z_{2}$ is a hyperKähler reduction $\mathcal{O} / / / S_{z_{2}}^{1}$ but now with respect to the $i$ 's level set of the momentum map. In other words, $\mathcal{H}\left(Z_{2}\right)=\mathcal{O}$.

In general we may assume that $c_{1}=0, c_{n}=1$ and the other $c$ 's are integers. Then we may change the action of the torus $\mathbb{T}^{n}$ such that the hyperKähler reduction is still diffeomorphic to $Z_{n}$ but the level of momentum map becomes $(0, \ldots, 0, i)$. For the sake of simplicity we put $n=3$. Indeed, then the system $(2.24)$ is equivalent to

$$
\begin{aligned}
y_{1} i \bar{y}_{1}+y_{2} i \bar{y}_{2}+y_{3} i \bar{y}_{3}+y_{4} i \bar{y}_{4} & =0 \\
\left(c_{2}-1\right) y_{1} i \bar{y}_{1}+y_{2} i \bar{y}_{2}-c_{2} y_{3} i \bar{y}_{3} & =0 \\
-y_{1} i \bar{y}_{1}+y_{3} i \bar{y}_{3} & =i,
\end{aligned}
$$


and the left-hand sides of the above equations are momentum maps of the following $\mathbb{T}_{w}^{3}=S_{w_{1}}^{1} \times S_{w_{2}}^{1} \times S_{w_{3}}^{1}$-action:

$$
\left(y_{1}, y_{2}, y_{3}, y_{4}\right) \mapsto\left(y_{1} w_{1} w_{2}^{c_{2}-1} \bar{w}_{3}, y_{2} w_{1} w_{2}, y_{3} w_{1} \bar{w}_{2}^{c_{2}} w_{3}, y_{4} w_{1}\right) .
$$

Moreover, there is a bijection $\mathbb{T}_{w}^{3} \rightarrow \mathbb{T}^{3}, z_{1}=w_{1}, z_{2}=w_{2}, z_{3}=w_{3} \bar{w}_{1}^{c_{1}}$ which respects the corresponding actions. It follows that $Z_{3}$ is a hyperKähler reduction of $\mathbb{H}^{4}$ by $\mathbb{T}_{w}^{3}$, which we can regard as being performed in two steps: first hyperKähler reduction $\hat{Z}_{3}$ of $\mathbb{H}^{4}$ by $S_{w_{1}}^{1} \times S_{w_{2}}^{1}$ with respect to the zero level of momentum map and then reduction of $\hat{Z}_{3}$ by $S_{w_{3}}^{1}$ with respect to $i$ 's level of momentum map. Thus $\hat{Z}_{3}$ has a hyperKähler potential and $\mathcal{H}\left(Z_{3}\right)=\hat{Z}_{3}$.

\subsection{Indeterminacy of function $v$ : further ex- amples}

As we have seen in Section 2.1.4 the function $v$ is defined by formula (2.13) only up to a constant and this has strong consequences as we will see below. Recall that the action of $S_{r}^{1}$ should be lifted from $\tilde{M}$ to $P$ such that its Killing vector field $Y_{1}$ equals to $\hat{K}_{r}+2 v K_{0}$ (see 2.7)). This implies that we are free to take $\tilde{v}=v+m / 2$ instead of $v$, where $m$ is an integer whenever $v+m / 2$ remains everywhere positive. However in this case one needs to modify the lifting of the $S_{r}^{1}$-action to $P$ to get that the Killing vector field is given by $\hat{K}_{r}+2 \tilde{v} K_{0}$. Therefore we get that the manifolds $\mathbb{H}^{*} \times_{S_{r}^{1}} P$ and $P / S_{r}^{1}$ again carry hyperKähler and quaternionic Kähler structures correspondingly, where the modified action of $S_{r}^{1}$ is implied. It turns out that a modification of the $S_{r}^{1}$-action can change the topology of the $\mathcal{H}$ and $\mathcal{Q}$ constructions.

Consider Example 2.2.1 again. Let us first take $n=1$ for the sake of simplicity. Then $P=\mu^{-1}(i)=\left\{\left(y_{1}, y_{2}\right) \in \mathbb{H} \mid y_{1} i \bar{y}_{1}+y_{2} i \bar{y}_{2}=i\right\}$ and the actions of $S_{0}^{1}$ and $S_{r}^{1}$ are given by right- and left- multiplications correspondingly. Since $T^{*} \mathbb{C P}^{1}$ is obtained as a hyperKähler quotient of $\mathbb{H}^{2}$ by $S_{0}^{1}$ it follows that the function $v$ is positive (explicitely, $v^{-1}=\left|y_{1}\right|^{2}+\left|y_{2}\right|^{2}$ ). Therefore we may consider $\tilde{v}=v+1 / 2$ and the modified action of $S_{r}^{1}$ takes a form

$$
z \cdot\left(z_{1}, z_{2}, w_{1}, w_{2}\right)=\left(z^{2} z_{1}, z^{2} z_{2}, w_{1}, w_{2}\right),
$$

where $y_{l}=z_{l}+w_{l} j, l=1,2$. Now as a consequence of the above observation we obtain the following statement.

Proposition 2.3.1. The total space of $T \mathbb{C P}^{1}(-1)=\mathcal{O}(1)$ carries a quaternionic Kähler structure. 
Proof. First observe that $\left(\mathbb{H}^{2} \backslash 0\right) / S_{r}^{1} \cong \mathbb{C P}_{\left[z_{1}: z_{2}\right]}^{1} \times \mathbb{R}_{>0} \times \mathbb{C}_{w_{1} w_{2}}^{2}$, where the projection onto $\mathbb{R}_{>0}$ is given by the function $\left(z_{1}, z_{2}, w_{1}, w_{2}\right) \mapsto\left|z_{1}\right|^{2}+\left|z_{2}\right|^{2}$ and the action $(2.28)$ is implied. The equation $\mu=i$, defining $P \subset \mathbb{H}^{2} \backslash 0$, can be rewritten as $\left|z_{1}\right|^{2}+\left|z_{2}\right|^{2}=1+\left|w_{1}\right|^{2}+\left|w_{2}\right|^{2}, z_{1} w_{1}+z_{2} w_{2}=0$. It follows that $P / S_{r}^{1} \cong\left\{\left(\left[z_{1}: z_{2}\right], w_{1}, w_{2}\right) \in \mathbb{C P}^{1} \times \mathbb{C}^{2} \mid z_{1} w_{1}+z_{2} w_{2}=0\right\}$. Now the statement follows from the Euler exact sequence: $0 \rightarrow \mathcal{O}(-1) \rightarrow \mathbb{C}^{2} \rightarrow$ $T \mathbb{C P}^{1}(-1) \rightarrow 0$.

The next natural task is to identify the Swann bundle of $\mathcal{O}(1)$. But first we would like to prove an auxiliary lemma.

Lemma 2.3.2. Let $P \rightarrow X$ be an $S^{1}$-principal bundle and $L \rightarrow X$ be the corresponding line bundle. Consider the following action of $S^{1}$ on $P \times \mathbb{C}$ : $z \cdot(p, w)=\left(p z^{r}, z^{s} w\right)$, where $r$ and $s$ are integers and $r$ is positive. Then

$$
(P \times \mathbb{C}) / S^{1} \cong L^{-s}
$$

Proof. Let $Q_{r, s}$ denote the space $P \times \mathbb{C}$ with the action of $S^{1}$ as in the statement of the lemma. Then we have an equivariant map $Q_{r, s} \rightarrow Q_{r, r s},(p, w) \mapsto$ $\left(p, w^{s}\right)$. Clearly it is surjective; although it is not injective, it descends to a bijective map of quotients $Q_{r, s} / S^{1} \rightarrow Q_{r, r s} / S^{1}$. But the last quotient is exactly $L^{-s}$.

Proposition 2.3.3. The total space of $2 \mathcal{O}(1) \oplus \mathcal{O}(-1)$ carries a hyperKähler structure and is a Swann bundle of $\mathcal{O}(1)$.

Proof. First, consider $\mathbb{H}^{*}$ with quaternionic variable $x$ as $\mathbb{C}^{2} \backslash 0$ with variables $w_{3}$ and $w_{4}: x=w_{3}+w_{4} j$. Similarly to the proof of Proposition 2.3.1. first consider a bigger space $\left(\mathbb{H}^{*} \times \mathbb{H}^{2}\right) / S_{r}^{1}$. Now the action has the form $z \cdot\left(z_{1}, z_{2}, w_{1}, w_{2}, w_{3}, w_{4}\right)=\left(z^{2} z_{1}, z^{2} z_{2}, w_{1}, w_{2}, \bar{z} w_{3}, z w_{4}\right)$. Further, since the Hopf fibration is the principal bundle of $\mathcal{O}(-1)$ and using Lemma 2.3.2 we get

$$
\left(\mathbb{H}^{*} \times \mathbb{H}^{2}\right) / S_{r}^{1} \cong \mathcal{O}(1) \oplus \mathcal{O}(-1) \oplus \mathbb{C}_{w_{1} w_{2}}^{2} \times \mathbb{R}_{>0},
$$

where the projection to $\mathbb{R}_{>0}$ is again given by the function $\left|z_{1}\right|^{2}+\left|z_{2}\right|^{2}$.

The condition $\left(z_{1}, z_{2}, w_{1}, w_{2}\right) \in P$ extracts $\mathcal{O}(1)$ out of $\mathbb{C}^{2} \times \mathbb{R}_{>0}$ in the same way as in the proof of Proposition 2.3.1.

The above analysis applies without changes for higher dimensions and the following proposition holds.

Proposition 2.3.4. The total space of $T \mathbb{C P}^{n}(-1)$ carries a quaternionic Kähler structure and $\mathcal{U}\left(T \mathbb{C P}^{n}(-1)\right)=\mathcal{O}(1) \oplus \mathcal{O}(-1) \oplus T \mathbb{C P}^{n}(-1)$. 
Finally, consider a general situation $\tilde{v}=v+m / 2$. In this case $P=$ $\left\{\left(y_{1}, \ldots, y_{n+1}\right) \mid y_{1} i \bar{y}_{1}+\cdots+y_{n+1} i \bar{y}_{n+1}=i\right\}$ and the modified action of $S_{r}^{1}$ becomes:

$$
\begin{aligned}
& z \cdot\left(z_{1}, \ldots, z_{n+1}, w_{1}, \ldots, w_{n+1}\right)= \\
& \quad=\left(z^{1+m} z_{1}, \ldots, z^{1+m} z_{n+1}, z^{1-m} w_{1}, \ldots, z^{1-m} w_{n+1}\right) .
\end{aligned}
$$

Theorem 2.3.5. Let $Q_{m}^{n}$ and $H_{m}^{n}$ denote the total spaces of $T \mathbb{C P}^{n}(m-2)$, $m \geq 1$ and $\mathcal{O}(1) \oplus \mathcal{O}(-1) \oplus T \mathbb{C P}^{n}(m-2)$ correspondingly. Then $Q_{m}^{n}$ is a quaternionic Kähler manifold, $H_{m}^{n}$ is a hyperKähler one and $\mathcal{U}\left(Q_{m}^{n}\right)=H_{m}^{n}$. In particular, $Q_{m}^{1}=\mathcal{O}_{\mathbb{P}^{1}}(m), m \geq 1$ are quaternionic Kähler manifolds, i.e. Einstein and self-dual.

\subsection{Kähler structure on $N_{0}$}

In contrast to a hyperKähler manifold, almost complex structures of a quaternionic Kähler manifold $N$ are defined only locally, i.e. we have a distinguished rank 3 subbbundle $\mathcal{I} \subset \operatorname{End}(T N)$ called a structure bundle, which locally admits a basis consisting of three almost complex structures with quaternionic relations. Since the metric induces an isomorphism $T N \cong T^{*} N$, one gets an embedding of $\mathcal{I}$ in $\Lambda^{2} T^{*} N$. Locally this is given by passing from an almost complex structure $I$ to the associated 2 -form $\omega_{I}(\cdot, \cdot)=g(\cdot, I \cdot)$. We will not distinguish between $\mathcal{I}$ and its image in $\Lambda^{2} T^{*} N$. An analogue of a momentum map can be defined in the quaternionic Kähler context, but now it will be a section of a structure bundle (see [31] for details).

Theorem 2.4.1. Let $N$ be a quaternionic Kähler manifold of positive scalar curvature. Suppose also that $N$ admits a quaternionic Kähler action of $S^{1}$ with momentum section $\mu_{N} \in \Gamma(\mathcal{I})$. Then $N_{0}=N \backslash\left\{\mu_{N}=0\right\}$ is a Kähler manifold.

Proof. Let $M$ be the Swann bundle of $N$. Then $M$ admits a hyperKähler action of $S^{1}$ [65]. Let $\mu=\mu_{1} i+\mu_{c} j: M \rightarrow \operatorname{Im} \mathbb{H}$ be its momentum map. Since the function $\mu_{c}: M \rightarrow \mathbb{C}$ is $I_{1}$-holomorphic, the submanifold $M_{c}=\{m \in$ $\left.M: \mu_{c}(m)=0\right\}$ has an induced Kähler structure. Further, $M_{c}^{+}=\left\{m \in M_{c}\right.$ : $\left.\mu_{1}(m)>0\right\}$ is an open submanifold of $M_{c}$. The group $S_{r}^{1}$ preserves $I_{1}$ and one may consider the Kähler reduction of $M_{c}^{+}$with respect to a non-zero value of the momentum map : $M_{c}^{+} / / S_{r}^{1} \cong M_{c}^{+} / \mathbb{C}_{r}^{*}$. It remains to observe that $M_{c}^{+}=\mu^{-1}(i) \times \mathbb{R}_{>0}=P \times \mathbb{R}_{>0}$ and therefore $M_{c}^{+} / \mathbb{C}_{r}^{*} \cong P / S_{r}^{1} \cong N_{0}$.

When a quaternionic Kähler manifold $N$ admits an action of $S^{1}$, one can normalize the momentum section $\mu_{N} \in \Gamma(\mathcal{I})$ and consider it as an almost 
complex structure $\hat{I}$ over $N_{0}$. It turns out that $\hat{I}$ is integrable and it is easy to see from the proof that it coincides with the complex structure implied by Theorem 2.4.1 (our proof of the above theorem itself represents an alternative proof of the integrability of $\hat{I}$ in case when $N$ has positive scalar curvature). In this form Theorem 2.4.1 was known earlier [7, 60. Although the complex structure $\hat{I}$ is a section of the structure bundle $\mathcal{I}$, the Kähler metric of $N_{0}$ must not coincide with the quaternionic Kähler one as we will see in the sequel. Note also that $\mathcal{I}$ does not admit a section which defines an integrable complex structure on the whole manifold $N$ (see [1] for extensive discussion of this phenomenon). Taking this into account, one may consider $N_{0}$ as "the largest" open submanifold of $N$ where it is still possible to choose an integrable complex structure.

Our next aim is to express the Kähler structure of $N_{0}$ similarly to the quaternionic Kähler one (see section 2.1.5).

Recall that $N_{0} \cong P / S_{r}^{1}=M_{c}^{+} / \mathbb{C}_{r}^{*}$. In order to get a metric and Kähler form on $N_{0}$ we have to express $N_{0}$ as a Kähler reduction, i.e. we have to fix a level set of momentum map and divide it by $S_{r}^{1} \subset \mathbb{C}_{r}^{*}$. In our case the momentum map of the $S_{r}^{1}$-action is nothing else but the hyperKähler potential $\rho$ (restricted to $M_{c}^{+}$). Recall also that we have an isomorphism (2.17) between $P$ and $Q=\rho^{-1}(-1 / 2) \cap M_{c}^{+}$. Further, one has $T . M_{c}=$ $\operatorname{span}\left(I_{2} K_{0}, I_{3} K_{0}\right)^{\perp} \subset T . M$ and $T . \rho^{-1}(-1 / 2)=\operatorname{span}\left(Y_{0}\right)^{\perp}$. It follows that T. $Q=\operatorname{span}\left(I_{2} K_{0}, I_{3} K_{0}, Y_{0}\right)^{\perp}$ because $Y_{0}$ is perpendicular to both $I_{2} K_{0}$ and $I_{3} K_{0}$ (see (2.7)). In particular $Y_{1} \in T Q$; this also follows from the fact that $S_{r}^{1}$ preserves $Q$. Further, the Kähler reduction procedure implies that T. $N_{0}$ is identified with $\operatorname{span}\left(Y_{1}\right)^{\perp} \subset T . Q$ and the Kähler form and metric are obtained as a restriction of the corresponding tensors to span $\left(Y_{1}\right)^{\perp}$. Remark that the quaternionic Kähler metric was obtained as the one induced on a different subbundle, namely on span $\left(Y_{1}, Y_{2}, Y_{3}\right)^{\perp} \subset T Q$.

Let $\mathrm{u} \in T_{p} P$. Then we may decompose $\mathrm{u}=\mathrm{u}^{\prime}+\psi(\mathrm{u}) Y_{1}$, where $\mathrm{u}^{\prime}$ is orthogonal to $Y_{1}$. Now denote by $\Pi$ an orthogonal projector on $\operatorname{span}\left(Y_{1}\right)^{\perp}$ in $T Q$. Then for the Kähler metric $\hat{g}_{\mathrm{N}}$ we have:

$$
\begin{aligned}
& \hat{g}_{\mathrm{N}}(\mathrm{u}, \mathrm{v})=g\left(\Pi l_{*} \mathrm{u}, \Pi l_{*} \mathrm{v}\right)= \\
& \quad=g\left(\left(L_{\lambda(p)}\right)_{*} \mathrm{u}^{\prime}+d \lambda(\mathrm{u}) Y_{0}(\lambda(p) p),\left(L_{\lambda(p)}\right)_{*} \mathrm{v}^{\prime}+d \lambda(\mathrm{v}) Y_{0}(\lambda(p) p)\right)= \\
& \quad=\lambda^{2} g\left(\mathrm{u}-\psi(\mathrm{u}) Y_{1}+d \lambda(\mathrm{u}) Y_{0}, \mathrm{v}-\psi(\mathrm{v}) Y_{1}+d \lambda(\mathrm{v}) Y_{0}\right)= \\
& \quad=\lambda^{2}\left(g(\mathrm{u}, \mathrm{v})-\psi(\mathrm{u}) g\left(Y_{1}, \mathrm{v}\right)-\psi(\mathrm{v}) g\left(Y_{1}, \mathrm{u}\right)+d \lambda(\mathrm{u}) g\left(Y_{0}, \mathrm{v}\right) d \lambda(\mathrm{v}) g\left(Y_{0}, \mathrm{u}\right)\right) .
\end{aligned}
$$

As we already know $g(\mathrm{u}, \mathrm{v})=\left(\tilde{g}+v^{-1} \xi^{2}\right)(\mathrm{u}, \mathrm{v})$. By the definition of $\psi$ one has $g\left(Y_{1}, \mathrm{v}\right)=(4 v+w) \psi(\mathrm{v})$. Further, $g\left(Y_{0}, \mathrm{v}\right)=g\left(-I_{1} K_{r}-2 v I_{1} K_{0}, \hat{\mathrm{v}}+\xi(\mathrm{v}) K_{0}\right)=$ 
$\imath_{K_{r}} \tilde{\omega}_{1}(\mathrm{v})$. Therefore we obtain $\hat{g}_{\mathrm{N}}=\lambda^{2}\left(\tilde{g}+v^{-1} \xi^{2}-(4 v+w) \psi^{2}+d \lambda \odot \imath_{K_{r}} \tilde{\omega}_{1}\right)$. Since $d \lambda=(4 v+w)^{-3 / 2} \imath_{K_{r}} \tilde{\omega}_{1}$ we may finally write

$$
\hat{g}_{\mathrm{N}}=\frac{1}{4 v+w} \tilde{g}+\frac{1}{v(4 v+w)} \xi^{2}-\psi^{2}+\frac{1}{(4 v+w)^{5 / 2}}\left(\imath_{K_{r}} \tilde{\omega}_{1}\right)^{2} .
$$

The Kähler form $\hat{\omega}_{N}$ may be obtained in a similar manner. Indeed,

$$
\begin{aligned}
\hat{\omega}_{N}(\mathrm{u}, \mathrm{v}) & =\omega_{1}\left(\Pi l_{*} \mathrm{u}, \Pi l_{*} \mathrm{v}\right)= \\
& =\omega_{1}\left(\left(L_{\lambda(p)}\right)_{*} \mathrm{u}^{\prime}+d \lambda(\mathrm{u}) Y_{0}(l(p)),\left(L_{\lambda(p)}\right)_{*} \mathrm{v}^{\prime}+d \lambda(\mathrm{v}) Y_{0}(l(p))\right)= \\
& =\omega_{1}\left(\left(L_{\lambda(p)}\right)_{*} \mathrm{u}^{\prime},\left(L_{\lambda(p)}\right)_{*} \mathrm{v}^{\prime}\right)= \\
& =\lambda^{2} \omega_{1}\left(\mathrm{u}-\psi(\mathrm{u}) Y_{1}, \mathrm{v}-\psi(\mathrm{v}) Y_{1}\right) .
\end{aligned}
$$

Since $\omega_{1}(\mathrm{u}, \mathrm{v})=\tilde{\omega}_{1}(\mathrm{u}, \mathrm{v})$ and $\omega_{1}\left(Y_{1}, \mathrm{u}\right)=g\left(\hat{K}_{r}+2 v K_{0}, I_{1} \hat{\mathrm{u}}+\xi(\mathrm{u}) I_{1} K_{0}\right)=$ $\imath_{K_{r}} \tilde{\omega}_{1}(\mathrm{u})$, we obtain the Kähler form as

$$
\hat{\omega}_{N}=\frac{1}{4 v+w}\left(\tilde{\omega}_{1}-\psi \wedge \imath_{K_{r}} \tilde{\omega}_{1}\right)
$$

Remark 2.4.2. As we have already remarked, we may regard the form $\psi$ as a connection on the $S_{r}^{1}$-principal bundle $P \rightarrow N_{0}$. Lets compute its curvature. We have

$$
F_{\psi}=-\frac{1}{(4 v+w)^{2}}(4 d v+d w) \wedge\left(2 \xi+\imath_{K_{r}} \tilde{g}\right)+\frac{1}{4 v+w}\left(2 d \xi+d \imath_{K_{r}} \tilde{g}\right)
$$

It follows from equations 2.11 and 2.12 that

$$
F_{\psi}=-\frac{2}{4 v+w}\left(\imath_{K_{r}} \tilde{\omega}_{1} \wedge \psi+\tilde{\omega}_{1}\right)=-2 \hat{\omega}_{N}
$$

This observation provides an "internal" interpretation of the Kähler form $\hat{\omega}_{N}$ in the following sense. Let $N$ be a quaternionic Kähler manifold with positive scalar curvature and $F \rightarrow N$ be the principal $S O(3)$ bundle associated to the structure bundle $\mathcal{I}$. Observe that $F$ is equipped with the natural connection induced by the Levi-Civita one. Suppose also that $N$ admits a quaternionic Kähler action of the circle and denote by $\mu_{N}$ its momentum section. As it was explained above one can think about $\mu_{N}$ on $N_{0}=N \backslash\left\{\mu_{N}=0\right\}$ as a section of $F$ (restricted to $N_{0}$ ). This means that we get $S^{1}$-subbundle $Q$ of $F$. The curvature of the induced connection $\psi$ is a $-1 / 2-$ multiple of the Kähler form $\hat{\omega}_{N}$. 


\section{Chapter 3}

\section{Nonlinear Dirac operator and harmonic spinors}

This chapter is devoted to a nonlinear generalization of the celebrated Dirac operator. In the preparatory Section 3.1 we discuss some representations of $\operatorname{Spin}^{c}(4)$. The results of Section 3.1 will be used in the sequel. In Section 3.2 we define a nonlinear generalization of the Dirac operator following [59] and study its properties. The nonlinear Dirac operator $\mathcal{D}$ acts on sections $u$ of a certain fibre bundle, where a fibre is a hyperKähler manifold $M$ admitting $S p(1)$ permuting and $S^{1}$ triholomorphic actions. When the Dirac operator is applied to a section $u$ one obtains a section of some vector bundle and therefore harmonic spinors are well-defined. We show that harmonic spinors are analogous to antiholomorphic sections of complex geometry (see Lemma 3.2.15 and its corollary). These results are of fundamental importance and will be used below as well as in Chapter 4 .

In Section 3.3 we study properties of solutions to the Cauchy-RiemannFueter equation (3.11), which is a quaternionic analogue of the CauchyRiemann equation in the complex geometry, and their relation to harmonic spinors. In particular, we prove that solutions to the Cauchy-RiemannFueter equation are exactly those maps whose differential has vanishing triholomorphic component (Proposition 3.3.10). These results are extended for maps between quaternionic Kähler manifolds and more generally for sections of vector bundles with quaternionic structures.

In the last section we study the case when the action of $S p(1)$ can be extended to the permuting action of $\mathbb{H}^{*}$. Combining our previous results with Swann's construction we get relations between earlier studied objects (harmonic spinors, aholomorphic maps and sections). These objects were defined earlier by different authors (and under a variety of names), however the properties and relations between them are new. 


\subsection{Spinor representations}

Recall that by $W$ we denote the standard $S U(2)$-representation on $\mathbb{C}^{2}$ or equivalently the $S p(1)$-representation on $\mathbb{H}$ by multiplication on the left. Further, $W^{ \pm}$denote $\operatorname{Spin}^{c}(4)$-representations on $\mathbb{H}$ given by $\left(\left[q_{+}, q_{-}, \lambda\right], h\right) \mapsto$ $q_{ \pm} h \lambda$ and $\mathbb{R}^{4}$ denotes the representation of the same group on the same space given by $\left(\left[q_{+}, q_{-}, \lambda\right], h\right) \mapsto q_{-} h \bar{q}_{+}$.

Any quaternionic module $V$ can be considered as a real vector space equipped with three complex structures $\left(I_{1}, I_{2}, I_{3}\right)$ with quaternionic relations. Consequently, any such vector space can be regarded as being equipped with a standard representation of $S p(1)$, namely $q=q_{0}+q_{1} i+q_{2} j+q_{3} k \equiv$ $q_{0} \mathrm{Id}+q_{1} I_{1}+q_{2} I_{2}+q_{3} I_{3}$.

Proposition 3.1.1. Let $V$ be a quaternionic module and denote by $V_{I_{1}}$ the same vector space but considered as a complex vector space with respect to the complex structure $I_{1}$. Under the isomorphism

$$
V_{\mathbb{C}}=\mathbb{C} \otimes V \cong \mathbb{H} \otimes_{\mathbb{C}} V_{I_{1}}, \quad z \otimes v \mapsto z \otimes v-j z \otimes I_{2} v
$$

the natural action of $S p(1)$ becomes multiplication on the left:

$$
z \otimes q \cdot v \mapsto q z \otimes v-q j z \otimes I_{2} v,
$$

i.e. we have the isomorphism of representations $V_{\mathbb{C}} \cong W \otimes_{\mathbb{C}} V_{I_{1}}$.

Remark 3.1.2. The complex tensor product in the statement above is defined via the relation $h i \otimes_{\mathbb{C}} v=h \otimes_{\mathbb{C}} I_{1} v$.

Proof. It is sufficient to check the statement for $q=i, j, k$. Indeed, one has

$z \otimes I_{1} v \mapsto z \otimes I_{1} v-j z \otimes I_{2} I_{1} v=i z \otimes v+j z i \otimes I_{2} v=i\left(z \otimes v-j z \otimes I_{2} v\right) ;$

$z \otimes I_{2} v \mapsto z \otimes I_{2} v-j z \otimes I_{2} I_{2} v=z \otimes I_{2} v+j z \otimes v=j\left(z \otimes v-j z \otimes I_{2} v\right) ;$

$z \otimes I_{3} v \mapsto z \otimes I_{1} I_{2} v-j z \otimes I_{1} v=i z \otimes I_{2} v-j z i \otimes v=k\left(z \otimes v-j z \otimes I_{2} v\right)$.

Corollary 3.1.3. Suppose we are given a representation of the group $S p(1) \times_{\{ \pm 1\}} S^{1} \cong U(2)$ on a quaternionic module $V$ such that the action of $S p(1) \subset S p(1) \times_{\{ \pm 1\}} S^{1}$ is standard and the action of the subgroup $S^{1}$ is quaternionic. Further, assume that the representation of $\operatorname{Spin}^{c}(4)$ on $V$ is induced via the homomorphism

$$
\operatorname{Spin}^{c}(4) \longrightarrow \operatorname{Spin}^{c}(4) / S p_{-}(1) \cong U_{+}(2) .
$$

Then the isomorphism $V_{\mathbb{C}} \cong \mathbb{H} \otimes_{\mathbb{C}} V$ induces an isomorphism of representations

$$
V_{\mathbb{C}} \cong W^{+} \otimes_{\mathbb{C}} V^{\prime}
$$

where $V^{\prime}$ is a representation of $S^{1}$ on $V$. 
Clifford multiplication First recall (see (1.4)) that one can consider the standard representation $\mathbb{R}^{4}$ of $S O(4)$ as being induced by $\operatorname{Spin}^{c}(4)$. Then under the hypotheses of Corollary 3.1 .3 we can define the following homomorphism of representations

$$
\mathbb{R}^{4} \otimes V_{\mathbb{C}} \cong \mathbb{R}^{4} \otimes W^{+} \otimes V^{\prime} \longrightarrow W^{-} \otimes V^{\prime}
$$

given by the formula:

$$
x \otimes(z \otimes v) \mapsto x z \otimes v-x j z \otimes I_{2} v .
$$

Observe also that homomorphism (3.2) induces a homomorphism between the real parts of the corresponding representations:

$$
\mathbb{R}^{4} \otimes V=\left[\mathbb{R}^{4} \otimes V_{\mathbb{C}}\right]_{r} \longrightarrow\left[W^{-} \otimes V^{\prime}\right]_{r}
$$

Remark that if $V=W^{+}$homomorphism (3.4) is the well-known Clifford multiplication:

$$
\mathbb{R}^{4} \otimes W^{+} \longrightarrow W^{-} .
$$

Therefore we will also call homomorphism (3.4) a Clifford multiplication. Slightly abusing notations, we will refer to its complexification $(3.2)$ also as Clifford multiplication if it will be clear from the context which homomorphism is implied.

\subsection{The Nonlinear Dirac operator}

Pick a $\operatorname{Spin}^{c}(4)$-structure $P_{\text {Spin }^{c}} \stackrel{\pi}{\longrightarrow} X$ over a four-dimensional oriented Riemannian manifold $\left(X, g_{X}\right)$. Fix also a hyperKähler manifold $M^{4(n+1)}$ equipped with an action of the group $U(2) \cong S p(1) \times_{ \pm 1} S^{1}$ with the following properties: the action of $S p(1)$ is permuting while the action of $S^{1}$ is hyperKähler.

Remark 3.2.1. There is a certain freedom in choice of complex structures on a hyperKähler manifold. Indeed, the space of triples of complex structures compatible with the preferred one is naturally isomorphic to $S O(3)$. However the requirement that the action of $S p(1)$ is permuting eliminates this freedom. From now on $\left(I_{1}, I_{2}, I_{3}\right)$ denotes the complex structures of $M$ such that the action of $S p(1)$ is permuting with respect to this triple.

The homomorphism (3.1) defines an action of $\operatorname{Spin}^{c}(4)$ on $M$. Denote by $K_{\xi}^{M}$ its Killing vector field corresponding to a Lie algebra element $\xi \in \mathfrak{s p i n} \mathfrak{n}^{\mathfrak{c}}(4)$. Further, define $\mathrm{M}$ to be a fibre bundle over $X$ with fibre $M$ :

$$
\mathrm{M}=P_{\text {Spinc }^{c}} \times_{\operatorname{Spin}^{c}(4)} M .
$$


We consider equivariant maps $u: P_{\text {Spin }^{c}} \rightarrow M$ as an analogue of spinors; one can also think about $u$ as a section of $\mathrm{M} \rightarrow X$. The space of all equivariant maps will be denoted by $\operatorname{Map}^{\operatorname{Spin}^{c}(4)}\left(P_{\text {Spin }^{c}}, M\right)$.

Next, pick a connection $a$ on a principal $S^{1}$-bundle $P_{d e t}=P_{\text {Spinc }} / \operatorname{Spin}(4)$, which is called a determinant bundle. Together with the Levi-Civita connection it equips $P_{\text {Spinc }}$ with a connection $\hat{a}$.

Covariant derivative. To any spinor $u$ one can associate an equivariant map $\hat{a} u: T P_{\text {Spin }^{c}} \rightarrow T M$ : if $\mathrm{v} \in T_{p} P_{\text {Spin }^{c}}$ then $\hat{a}(\mathrm{v}) \in \mathfrak{s p i n}^{\mathfrak{c}}(4)$ and we can put $\hat{a} u(\mathrm{v})=K_{\hat{a}(\mathrm{v})}^{M}(u(p)) \in T_{u(p)} M$. A covariant derivative of the spinor $u$ is then defined by

$$
\nabla^{a} u=u_{*}+\hat{a} u \in \operatorname{Map}^{\operatorname{Spin}^{c}(4)}\left(T P_{\text {Spin }^{c}}, T M\right) .
$$

It is easy to see that the above expression vanishes on the vertical vectors and this means that the covariant derivative can be regarded as an operator

$$
\nabla^{a}: \Gamma(\mathrm{M}) \rightarrow \Gamma\left(T^{*} X \otimes \pi_{!}\left(u^{*} T M\right)\right),
$$

where $\pi_{!}\left(u^{*} T M\right) \rightarrow X$ denotes the factor of $u^{*} T M \rightarrow P_{S p i n^{c}}$ by the group action.

Remark 3.2.2. Strictly speaking, the operator $\nabla^{a}$ is not well-defined, since its image depends on the element of the domain. However one can define $\nabla^{a}$ as a section of a certain vector bundle as follows. Consider an evaluation map ev $: \operatorname{Map}^{\operatorname{Spin}^{c}(4)}\left(P_{\text {Spin }^{c}}, M\right) \times P_{\text {Spin }^{c}} \rightarrow M,(u, p) \mapsto u(p)$. Then one gets the following diagram

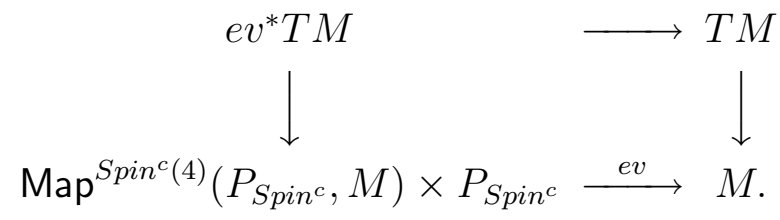

Dividing the first column by the group action one gets a vector bundle $\mathcal{E}$ over an the infinite dimentional space $\operatorname{Map}^{\operatorname{Spin}^{c}(4)}\left(P_{\text {Spin }^{c}}, M\right) \times X$; the restriction of $\mathcal{E}$ to $\{u\} \times X$ coincides with $\pi_{!}\left(u^{*} T M\right)$. Then $\nabla^{a}$ is well-defined as a section of $\mathcal{E} \rightarrow \operatorname{Map}^{\operatorname{Spin}^{c}(4)}\left(P_{\text {Spin }^{c}}, M\right) \times X$.

In order to keep the exposition clear, we will not keep to the above formalism of vector bundles over infinite-dimensional spaces.

Dirac operator. Now by the definition of a hyperKähler manifold the tangent bundle $T M$ carries a natural structure of a quaternionic module. Moreover the requirement that the action of $S p(1)$ is permuting implies that the induced action is the standard one. It follows from Proposition 3.1.1 that

$$
T_{\mathbb{C}} M \cong \mathbb{H} \otimes E^{\prime},
$$


where $E^{\prime}$ coincides with $T M$ as a vector bundle but the action of $S p_{+}(1) \subset$ $\operatorname{Spin}^{c}(4)$ is trivial; on the contrary the action of $U_{+}(2)$ on the first factor is standard. Therefore

$$
\pi_{!}\left(u^{*} T_{\mathbb{C}} M\right) \cong W^{+} \otimes E,
$$

where $E=\pi_{!}\left(u^{*} E^{\prime}\right)$ and by $W^{+}$we denoted the positive spinor bundle over $X$ corresponding to the representation denoted by the same letter. It follows from (3.4) that we have a map

$$
C l: T^{*} X \otimes \pi_{!}\left(u^{*} T M\right) \longrightarrow\left[W^{-} \otimes E\right]_{r} .
$$

With this understood, following [59] we give the following definition.

Definition 3.2.3. The first order differential operator given by the sequence

$$
\mathcal{D}_{a}: \Gamma(\mathrm{M}) \stackrel{\nabla^{a}}{\longrightarrow} \Gamma\left(T^{*} X \otimes \pi_{!}\left(u^{*} T M\right)\right) \stackrel{C l}{\longrightarrow} \Gamma\left(\left[W^{-} \otimes E\right]_{r}\right)
$$

is called a (generalized) Dirac operator.

Remark 3.2.4. When $M$ is a flat hyperKähler manifold $\mathbb{H}$ we recover the usual (linear) Dirac operator $\mathcal{D}_{a}: \Gamma\left(W^{+}\right) \rightarrow \Gamma\left(W^{-}\right)$.

Remark 3.2.5. One can define rigorously the generalized Dirac operator as a section of a vector bundle over an infinite-dimensional space in the same vane as we defined the covariant derivative in Remark 3.2.2. Indeed, it remains to note that the target of the Dirac operator $\left[W^{-} \otimes E\right]_{r}$ is a vector bundle over $\operatorname{Map}^{\operatorname{Spin}^{c}(4)}\left(P_{\text {Spin }^{c}}, M\right) \times X$.

Remark 3.2.6. The Dirac operator (3.5) is a nonlinear Fredholm map [59].

Remark 3.2.7. When the source manifold carries a Spin(4) structure one may define a Dirac operator with coefficients in $M$ by the above described scheme (see also Example 3.2.8). In that case $M$ is required to carry a permuting action of $S p(1)$, however a triholomorphic one of $S^{1}$ is not necessary for the definition and the resulting Dirac operator is determined by the LeviCivita connection.

Example 3.2.8 (SO-Dirac operator). Below we consider the Dirac operator in case when the target manifold $M$ admits a permuting $S O(3)$ action. For the sake of simplicity we take the most basic example $\mathbb{H}^{*} / \pm 1$.

Conventions. Take $W_{0}=\mathbb{H}^{*} / \pm 1$ as a hyperKähler manifold $M$. It is convenient in this example to take the hyperKähler structure on $W_{0}$ induced by the left quaternionic structure on $\mathbb{H}$. Then a permuting action of $S O(3) \cong$ $S p(1) / \pm 1$ is given by

$$
([q],[m]) \mapsto[q m]
$$


Further,

$$
T W_{0}=\left(\mathbb{H}^{*} \times \mathbb{H}\right) / \pm 1=\left\{[m, h]=[-m,-h] \mid m \in \mathbb{H}^{*}, h \in \mathbb{H}\right\}
$$

and the induced action on $T W_{0}$ is the following

$$
([q],[m, h]) \mapsto[q m, q h] .
$$

Consider the group $S O(4) \cong S p_{+}(1) \cdot S p_{-}(1)=\left(S p_{+}(1) \times S p_{-}(1)\right) / \pm 1$ (see Section 1.3). Consequently we have a homomorphism

$$
S O(4) \rightarrow S p_{+}(1) / \pm 1 \cong S O_{+}(3)
$$

and it defines an action of $S O(4)$ on $W_{0}$, which in turn induces an action of $S O(4)$ on $T W_{0}$.

Remark 3.2.9. We have also another action of the group $S O(4)$ on $T W_{0}$, namely

$$
\left(\left[q_{+}, q_{-}\right],[m, h]\right) \mapsto\left[q_{+} m, q_{-} h\right] .
$$

Note, that this action is not induced from the one on $W_{0}$.

Clifford multiplication. Let $X^{4}$ be an oriented Riemannian manifold and $\pi^{s o}: P_{s o} \rightarrow X$ be its principal $S O(4)$ bundle of orthonormal oriented frames. Consider an equivariant map $\psi: P_{s o} \longrightarrow W_{0}^{+}$or equivalently a section of a fibre bundle $\mathcal{W}_{0}^{+}=P_{s o} \times_{S O(4)} W_{0}^{+}$, where the sign " + " is used to keep in mind that the action of $S O(4)$ is induced by $S_{+}(3)$.

Remark 3.2.10. If the source manifold $X$ is Spin, then $\mathcal{W}_{0}^{+}=\left(W^{+} \backslash 0\right) / \pm 1$. Notice that even if $X$ is not Spin, the bundle $\mathcal{W}_{0}^{+}$does exist.

Now consider an equivariant vector bundle $\psi^{*} T W_{0}^{+} \longrightarrow P_{s o}$ which gives rise to $E^{+}=\pi_{!}^{s o}\left(\psi^{*} T W_{0}^{+}\right)$. Recall that there exists another action $(3.8)$ of $S O(4)$ on $T W_{0}$, which we now prefer to denote by $T W_{0}^{-}$. Consequently, there exists a vector bundle $E^{-}=\pi_{!}^{s o}\left(\psi^{*} T W_{0}^{-}\right)$.

One can think about $E^{+}$as a vector bundle of classes $[p, \xi]$, where $p \in$ $P_{s o}, \xi \in T_{\psi(p)} W_{0}$ such that $[p, \xi] \equiv\left[p g, g^{-1} \xi\right], g \in S O(4)$, i.e.

$$
[p,[\psi(p), h]] \equiv\left[p g,\left[\bar{q}_{+} \psi(p), \bar{q}_{+} h\right]\right], \quad g=\left[q_{+}, q_{-}\right] .
$$

Similarly, $E^{-}$is a vector bundle of classes $[p,[\psi(p), h]]$ such that

$$
[p,[\psi(p), h]] \equiv\left[p g,\left[\bar{q}_{+} \psi(p), \bar{q}_{-} h\right]\right], \quad g=\left[q_{+}, q_{-}\right] .
$$

Consider also $T X \cong T^{*} X$ in the same manner:

$$
[p, \mathrm{v}] \equiv\left[p g, \bar{q}_{-} \mathrm{v} q_{+}\right], \quad g=\left[q_{+}, q_{-}\right], \quad \mathrm{v} \in \mathbb{H} .
$$


Then we can define a variant of the Clifford multiplication $\mathrm{Cl}: T^{*} X \otimes$ $E^{+} \longrightarrow E^{-}$by the formula

$$
[p, \mathrm{v}] \otimes[p,[\psi(p), h]] \mapsto[p,[\psi(p), \mathrm{v} h]] .
$$

Indeed, take $g \in S O(4), g=\left[q_{+}, q_{-}\right]$. Then the following computation

$$
\left[p g, \bar{q}_{-\mathrm{v}} q_{+}\right] \otimes\left[p g,\left[\bar{q}_{+} \psi(p), \bar{q}_{+} h\right]\right] \mapsto\left[p g,\left[\bar{q}_{+} \psi(p), \bar{q}_{-} \mathrm{v} h\right]\right]=[p,[\psi(p), \mathrm{v} h]]
$$

shows that the Clifford multiplication 3.9 is well-defined.

Dirac Operator. After the Clifford multiplication is defined, we can also construct a Dirac operator. Indeed, consider the space of $S O(4)$-equivariant maps $\operatorname{Map}^{S O(4)}\left(P_{s o}, W_{0}^{+}\right)$or, equivalently, the space of sections of the associated bundle $\mathcal{W}_{0}^{+}=P_{\text {so }} \times_{S O(4)} W_{0}^{+}$.

Now the bundle $P_{s o}$ is equipped with the Levi-Civita connection $\nabla^{L C}$. Hence the following sequence defines a Dirac operator:

$$
\mathcal{D}: \Gamma\left(\mathcal{W}_{0}^{+}\right) \stackrel{\nabla^{L C}}{\longrightarrow} \Gamma\left(T^{*} X \otimes E^{+}\right) \stackrel{C l}{\longrightarrow} \Gamma\left(E^{-}\right) .
$$

Remark 3.2.11. We can also consider the operator 3.10 as a particular case of the Definition 3.2.3. Indeed, this corresponds to the manifold $M=\mathbb{H}^{*} / \pm 1$ and the trivial action of $S^{1}$. The above calculations show that it is independent of the other choices and in particular it is canonically defined by the $S O(4)-$ structure of $X$.

Remark 3.2.12. Consider a local chart $U$ of $X$ and pick a trivialization of $P_{s o}$ over $U$. In this trivialization an equivariant map $\psi$ can be written as $\psi_{U}: U \longrightarrow \mathbb{H}$. Then

$$
\mathcal{D} \psi_{U}=\frac{\partial \psi_{U}}{\partial x_{0}}+i \frac{\partial \psi_{U}}{\partial x_{1}}+j \frac{\partial \psi_{U}}{\partial x_{2}}+k \frac{\partial \psi_{U}}{\partial x_{3}}+(0 \text {-order terms }) .
$$

Remark 3.2.13 (Twisted SO-Dirac operator). Again assume that the target hyperKähler manifold admits a permuting $S O(3)$-action. Pick a principal $G$-bundle $\pi^{G}: P_{G} \rightarrow X$ over the source manifold $X$ with a connection $a$ and put $P=P_{\text {so }} \times P_{G} \stackrel{\pi}{\longrightarrow} X$. Note that the bundle $P$ is equipped with the connection determined by the Levi-Civita connection and $a$. Assume further that the action of $G$ is triholomorphic and that it commutes with the $S O(3)$ action. As in Example 3.2.8 we take $M^{*}=\mathbb{H}^{*} / \pm 1$ as the target manifold (we reserve the symbol $W_{0}$ for the $S O(3)$-action (3.6) ). Finally, denote by $E^{G}$ the bundle $T M^{*}$ with the induced action of $G$.

It is easy to see that the scheme of Example 3.2 .8 also applies in this case. Equivalently, one may complexify the tangent bundle of $M^{*}$ and apply the 
scheme of Definition 3.2 .3 since $\pi_{!}\left(u^{*} T_{\mathbb{C}} M^{*}\right) \cong \mathcal{W}_{0}^{+} \otimes \pi_{!}^{G}\left(u^{*} E^{G}\right)$. In any case we get a Dirac operator twisted by $\pi_{!}^{G}\left(u^{*} E^{G}\right)$ :

$$
\mathcal{D}_{a}: \Gamma(\mathrm{M}) \rightarrow \Gamma\left(E^{-} \otimes \pi_{!}^{G}\left(u^{*} E^{G}\right)\right) .
$$

Definition 3.2.14. A spinor $u \in \Gamma(\mathrm{M})$ such that $\mathcal{D}_{a} u=0$ is called harmonic.

Lemma 3.2.15. Pick $p \in P_{\text {Spin }^{c}}, \pi(p)=x$. Since there exists a projection $P_{\text {Spinc }} \rightarrow P_{\text {so }}$, one gets a basis $\left(\mathrm{v}_{0}, \mathrm{v}_{1}, \mathrm{v}_{2}, \mathrm{v}_{3}\right)$ of $T_{x} X$. Further, let $u \in \operatorname{Map}^{\operatorname{Spin}^{c}(4)}\left(P_{\text {Spin }^{c}}, M\right)$ and put $\mathrm{w}_{i}=u_{*}\left(\tilde{\mathrm{v}}_{i}\right) \in T_{u(p)} M$, where $\tilde{\mathrm{v}}_{i} \in T_{p} P_{\text {Spin }^{c}}$ denotes a horizontal lift of $\mathrm{v}_{i}$ with respect to the connection $\hat{a}$. Then the harmonicity of the spinor $u$ at the point $x$ is equivalent to the following equation:

$$
\mathrm{w}_{0}+I_{1} \mathrm{w}_{1}+I_{2} \mathrm{w}_{2}+I_{3} \mathrm{w}_{3}=0 .
$$

Proof. The complexification of the Clifford multiplication $T^{*} X \otimes \pi_{!}\left(u^{*} T M\right) \rightarrow$ $\left[W^{-} \otimes E\right]_{r}$ can be described by the following sequence

$$
T . X \otimes T . M \longrightarrow T . X \otimes(\mathbb{C} \otimes T . M) \longrightarrow T . X \otimes W^{+} \otimes E \longrightarrow W^{-} \otimes E .
$$

The basis $\left(\mathrm{v}_{0}, \mathrm{v}_{1}, \mathrm{v}_{2}, \mathrm{v}_{3}\right)$ provides an isomorphism $T_{x} X \cong \mathbb{H}$. Then the maps in the sequence above are given explicitly (see (3.3) ) by

$\mathrm{v} \otimes \mathrm{w} \mapsto \mathrm{v} \otimes(1 \otimes \mathrm{w}) \mapsto \mathrm{v} \otimes\left(1 \otimes \mathrm{w}-j \otimes I_{2} \mathrm{w}\right) \mapsto \mathrm{v} \cdot 1 \otimes \mathrm{w}-\mathrm{v} \cdot j \otimes I_{2} \mathrm{w}$.

According to the definition of the Dirac operator, take $\mathrm{v}=1, w=w_{0} ; \mathrm{v}=$ $i, w=w_{1} ; \cdots$ and then sum up the result:

$$
\begin{gathered}
\left(1 \otimes \mathrm{w}_{0}-j \otimes I_{2} \mathrm{w}_{0}\right)+\left(i \otimes \mathrm{w}_{1}-k \otimes I_{2} \mathrm{w}_{1}\right)+ \\
+\left(j \otimes \mathrm{w}_{2}+1 \otimes I_{2} \mathrm{w}_{2}\right)+\left(k \otimes \mathrm{w}_{3}+i \otimes I_{2} \mathrm{w}_{3}\right)=0,
\end{gathered}
$$

After a simplification one obtains

$$
1 \otimes\left(\mathrm{w}_{0}+I_{1} \mathrm{w}_{1}+I_{2} \mathrm{w}_{2}+I_{3} \mathrm{w}_{3}\right)+j \otimes\left(-I_{2} \mathrm{w}_{0}+I_{3} \mathrm{w}_{1}+\mathrm{w}_{2}-I_{1} \mathrm{w}_{3}\right)=0 .
$$

But this is equivalent to equation (3.11.

Denote by $\mathcal{V}$ the vertical subbundle of $T P_{\text {Spin }^{c}}$, i.e. $\mathcal{V}$ consists of tangent vectors to fibers of $P_{\text {Spin }} \rightarrow X$. The connection $\hat{a}$ provides a decomposition $T P_{\text {Spin }^{c}}=\mathcal{V} \oplus \mathcal{H}$, where $\mathcal{H}$ is defined as the kernel of $\hat{a}$ and is called the horizontal subbundle. Since $\mathcal{H} \cong \pi^{*} T X$ we get a distinguished quaternionic structure $\left(J_{1}, J_{2}, J_{3}\right)$ on $\mathcal{H}$. Indeed, observe that we have a natural projection $P_{\text {Spin }} \rightarrow P_{\text {Spin }} / U_{-}(2)=P_{\Lambda_{+}^{2}}$, where the $S O(3)$-principal bundle $P_{\Lambda_{+}^{2}}$ is 
the principal bundle of $\Lambda_{+}^{2} T^{*} X$. With the help of metric on $X$, one can naturally identify the fibre of $P_{\Lambda_{+}^{2}}$ at each point $x \in X$ with the set of triples $\left(\mathrm{J}_{1}, \mathrm{~J}_{2}, \mathrm{~J}_{3}\right), \mathrm{J}_{l} \in \operatorname{End}\left(T_{x} X\right)$ such that $\mathrm{J}_{1} \mathrm{~J}_{2}=\mathrm{J}_{3}=-\mathrm{J}_{2} \mathrm{~J}_{1}$ and each $\mathrm{J}_{l}$ is an orthogonal operator preserving the orientation of $T_{x} X$. Although the operators $J_{l}$ are not necessarily globally defined, the projection map $P_{\text {Spin }} \rightarrow$ $P_{\Lambda_{+}^{2}}$ gives rise to a global quaternionic structure on $\mathcal{H}$.

One can also describe the quaternionic structure $\left(J_{1}, J_{2}, J_{3}\right)$ as follows. Let $p \in P_{\text {Spin }^{c}}$ and $\left(\tilde{\mathrm{v}}_{0}, \tilde{\mathrm{v}}_{1}, \tilde{\mathrm{v}}_{2}, \tilde{\mathrm{v}}_{3}\right)$ be as in Lemma 3.2.15. Then the quternionic structure is defined by the requirement $\tilde{\mathrm{v}}_{l}=-\bar{J}_{l} \tilde{\mathrm{v}}_{0}, l=1,2,3$.

Corollary 3.2.16. A map $u \in \operatorname{Map}^{\operatorname{Spin}^{c}(4)}\left(P_{\text {Spin }^{c}}, M\right)$ is a harmonic spinor if and only if it satisfies the following equation

$$
u_{*}-I_{1} u_{*} J_{1}-I_{2} u_{*} J_{2}-I_{3} u_{*} J_{3}=0 \text { on } \mathcal{H} \text {. }
$$

Proof. For any $\mathrm{v} \in T X$ one can choose $p \in P_{\text {Spinc }^{c}}$ such that the basis $\left(\mathrm{v}_{0}, \mathrm{v}_{1}, \mathrm{v}_{2}, \mathrm{v}_{3}\right)$ defined by $p$ satisfies $\mathrm{v}_{0}=\mathrm{v}$. According to the definition of $\left(J_{1}, J_{2}, J_{3}\right)$ one has $\tilde{\mathrm{v}}_{l}=-J_{l} \tilde{\mathrm{v}}_{0}=-J_{l} \tilde{\mathrm{v}}, l=1,2,3$. Now it follows from Lemma 3.2 .15 that

$$
u_{*} \tilde{\mathrm{V}}-I_{1} u_{*} J_{1} \tilde{\mathrm{v}}-I_{2} u_{*} J_{2} \tilde{\mathrm{v}}-I_{3} u_{*} J_{3} \tilde{\mathrm{v}}=0 .
$$

It remains to note that the above equation is valid for any $\mathrm{v} \in T X$.

The above corollary reveals a deep analogy between the Dirac operator and the $\partial$-operator of complex geometry. Let us first recall the definition of the $\partial$-operator.

Definition 3.2.17. Let $\left(Y, I_{Y}\right)$ and $\left(Z, I_{Z}\right)$ be complex manifolds. Assume that a Lie group $G$ acts holomorphically on $Z$ and pick a $G$-principal bundle $\pi^{G}: P_{G} \rightarrow Y$ with a connection $A$. Then the $\partial_{A}$-operator is given by the following sequence

$$
\partial_{A}: \Gamma\left(P_{G} \times_{G} Z\right) \stackrel{\nabla}{\longrightarrow} \Omega^{1}(Y) \otimes \Gamma\left(\pi_{!}^{G}\left(u^{*} T Z\right)\right) \rightarrow \Omega^{1,0}(Y) \otimes \Gamma\left(\pi_{!}^{G}\left(u^{*} T Z\right)\right),
$$

where the last arrow is induced by the projection $\Omega^{1}(Y) \rightarrow \Omega^{1,0}(Y)$.

We will suppress the dependence of $\partial_{A}$-operator on $A$ in notations, if the connection will be clear from the context.

Further, we also have a splitting of the tangent bundle of $P_{G}$ on vertical and horizontal parts: $T P_{G}=\mathcal{V} \oplus \mathcal{H}$. It is well-known (see for example [40] for the case of $\bar{\partial}$-operator) that $\partial u=0$ is equivalent to

$$
u_{*}-I_{Z} u_{*} I_{Y}=0 \quad \text { on } \mathcal{H} \text {. }
$$

We will further develop the analogy between Dirac and $\partial$ operators below.

\footnotetext{
${ }^{\text {a }}$ notice that $\left(I_{1}, I_{2}, I_{3}\right)$ is the distinguished triple on $M$; see Remark 3.2 .1 for details
} 
Remark 3.2.18. Under assumptions of Definition 3.2 .17 the operator $\bar{\partial}_{A}$ is defined by the sequence

$$
\bar{\partial}_{A}: \Gamma\left(P_{G} \times_{G} Z\right) \stackrel{\nabla}{\longrightarrow} \Omega^{1}(Y) \otimes \Gamma\left(\pi_{!}^{G}\left(u^{*} T Z\right)\right) \rightarrow \Omega^{0,1}(Y) \otimes \Gamma\left(\pi_{!}^{G}\left(u^{*} T Z\right)\right) .
$$

As we have already mentioned above, the condition $\bar{\partial}_{A} u=0$ is equivalent to $I_{Z} u_{*}=u_{*} I_{Y}$ on $\mathcal{H}$.

Convention 3.2.19. We say, that $u \in \operatorname{Map}^{G}\left(P_{G}, Z\right)$ is an equivariant holomorphic map with respect to the connection $A$, if it defines a holomorphic section of the associated bundle $P_{G} \times_{G} Z$, i.e. if $\bar{\partial}_{A} u=0$. Notice, that the source of an equivariant holomorphic map must not be a complex manifold.

\subsection{Aholomorphic maps}

Assume for a while that the source manifold $X$ is hyperKähler, that is it admits three global complex structures $\left(J_{1}, J_{2}, J_{3}\right)$. In particular the principal bundle of $\Lambda_{+}^{2} X$, is trivial (as well as the connection). Suppose $M$ admits a permuting $S O(3)$-action. Since $S O_{-}(3)$ acts trivially on $M$, we can identify spinors with the usual maps $u: X \rightarrow M$. We then obtain from Corollary 3.2 .16 that $u$ is harmonic iff

$$
u_{*}-I_{1} u_{*} J_{1}-I_{2} u_{*} J_{2}-I_{3} u_{*} J_{3}=0 .
$$

The maps satisfying the above equation are of particular importance in quaternionic geometry and we will study their properties below.

\subsubsection{Linear algebra: aquaternionic operators}

Let $\left(U, J_{1}, J_{2}, J_{3}\right)$ and $\left(V, I_{1}, I_{2}, I_{3}\right)$ be quaternionic modules. Define an operator $C: H_{\mathbb{R}}(U, V) \longrightarrow \operatorname{Hom}_{\mathbb{R}}(U, V)$ by the formula:

$$
C: A \mapsto I_{1} A J_{1}+I_{2} A J_{2}+I_{3} A J_{3} .
$$

Proposition 3.3.1. The operator $C$ has the following properties

(i) $C^{2}=3-2 C$;

(ii) the spectrum consists of 1 and -3 ;

(iii) the space $\operatorname{Hom}_{\mathbb{R}}(U, V)$ decomposes as a direct sum of eigenspaces of the operator $C$ :

$$
\operatorname{Hom}_{\mathbb{R}}(U, V)=B_{+} \oplus B_{-},
$$

where $B_{+}$corresponds to eigenvalue +1 and $B_{-}$to -3 . 
Proof. The following computation

$$
\begin{aligned}
C^{2}(A) & =I_{1}\left(I_{1} A J_{1}+I_{2} A J_{2}+I_{3} A J_{3}\right) J_{1}+ \\
& +I_{2}\left(I_{1} A J_{1}+I_{2} A J_{2}+I_{3} A J_{3}\right) J_{2}+ \\
& +I_{3}\left(I_{1} A J_{1}+I_{2} A J_{2}+I_{3} A J_{3}\right) J_{3}= \\
& =3 A-C(A)
\end{aligned}
$$

shows that the property (i) holds. The other two properties are direct consequences of the first one.

In the sequel we will use the following notations:

$$
\begin{aligned}
& B_{0}=\left\{A \in \operatorname{Hom}_{\mathbb{R}}(U, V) \mid A \circ J_{l}=I_{l} \circ A, l=1,2,3\right\}=H o m_{\mathbb{H}}(U, V) ; \\
& B_{r}=\left\{A \in \operatorname{Hom}_{\mathbb{R}}(U, V) \mid A \circ J_{r}=I_{r} \circ A, A \circ J_{l}=-I_{l} \circ A, l \neq r\right\},
\end{aligned}
$$

where $r$ runs through $1,2,3$.

Remark 3.3.2. There is no nontrivial operator which anticommutes with all complex structures. Indeed, if $A$ would be such operator, then one would have $C(A)=3 A$, but 3 is not an eigenvalue of $C$. The same argument shows that there is no nontrivial operator, which commutes with two complex structures and anticommutes with the third one.

Proposition 3.3.3. The following properties hold:

(i) the linear spaces $B_{r}$ are isomorphic: $B_{0} \cong B_{1} \cong B_{2} \cong B_{3}$;

(ii) one has a decomposition

$$
H_{\mathrm{O}} \mathrm{m}_{\mathbb{R}}(U, V)=B_{0} \oplus B_{1} \oplus B_{2} \oplus B_{3} .
$$

Proof. Observe that for example an isomorphism between $B_{0}$ and $B_{1}$ can be obtained as multiplication by $I_{1}$ on the left. This proves (i).

Further, for any operator $A \in \operatorname{Hom}_{\mathbb{R}}(U, V)$ we have a decomposition $A=A_{0}+A_{1}+A_{2}+A_{3}$ with

$$
\begin{aligned}
& A_{0}=\frac{1}{4}(A-C(A)), \\
& A_{1}=\frac{1}{4}\left(A-I_{1} A J_{1}+I_{2} A J_{2}+I_{3} A J_{3}\right), \\
& A_{2}=\frac{1}{4}\left(A+I_{1} A J_{1}-I_{2} A J_{2}+I_{3} A J_{3}\right), \\
& A_{3}=\frac{1}{4}\left(A+I_{1} A J_{1}+I_{2} A J_{2}-I_{3} A J_{3}\right),
\end{aligned}
$$

and each component lies in the corresponding subspace. Thus (ii) is also proven. 
Corollary 3.3.4. The decompositions (3.13) and (3.14) are related as follows:

$$
\begin{array}{ll}
B_{-}=B_{0}=\operatorname{Hom}_{\mathbb{H}}(U, V), & \operatorname{dim} B_{-}=4 m n \\
B_{+}=B_{1} \oplus B_{2} \oplus B_{3}, & \operatorname{dim} B_{+}=12 m n
\end{array}
$$

where we assume $\operatorname{dim}_{\mathbb{R}} U=4 m ; \operatorname{dim}_{\mathbb{R}} V=4 n$.

Proof. It easily follows from the definition of subspaces $B_{r}$ that $C(A)=-3 A$ for any $A \in B_{0}$ and $C(A)=A$ for any $A \in B_{r}, r=1,2,3$.

Definition 3.3.5. We say that an operator $A \in H_{0} m_{\mathbb{R}}(U, V)$ is aquaternionic, if $C(A)=A$, where $C$ is given by 3.12 .

Proposition 3.3.6. An operator $A \in H_{0} m_{\mathbb{R}}(U, V)$ is aquaternionic if and only if its quaternionic linear component vanishes.

Proof. Any operator $A \in H_{0} m_{\mathbb{R}}(U, V)$ can be decomposed as $A=A_{+}+A_{-}$ due to 3.13$)$. It follows from Corollary 3.3.4 that the equation $C(A)=A$ is equivalent to $A_{-}=0$ or in other words that the quternionic linear component of $A$ vanishes.

We suggest the term aquaternionic for operators $A$ satisfying $C(A)=$ $A$ since it reflects the property that $A$ has a vanishing quaternionic linear component.

Remark 3.3.7. If both spaces $U$ and $V$ carry an Euclidean scalar product then so does $\operatorname{Hom}_{\mathbb{R}}(U, V) \cong U^{*} \otimes V$. If the quaternionic structures are consistent with the Euclidean ones, i.e. if the complex structures are orthogonal operators, then decomposition (3.13) is in fact orthogonal. Therefore

$$
C(A)=A \Longleftrightarrow A \in\left(H_{\mathbb{H}}(U, V)\right)^{\perp}
$$

The space of linear homomorphisms as $S p(1)$-representation.

Again, let $\left(U, J_{1}, J_{2}, J_{3}\right)$ and $\left(V, I_{1}, I_{2}, I_{3}\right)$ be quaternionic modules. As we have already remarked $U$ and $V$ carry the standard representation of $S p(1)$, which will be denoted by the same letters. If we want to consider those spaces as trivial $S p(1)$-representations, we use $\tilde{U}$ and $\tilde{V}$. Further, $W \equiv W_{1}$ denotes the standard $S p(1)$-representation by left multiplication on the space of quaternions $\mathbb{H}$; then all other irreducible representations of $S p(1)$ are just symmetric powers of the standard representation [12]: $W_{n}=S^{n} W$. By $\mathbb{R}^{3}$ we denote the standard representation of $S O(3)$ or the induced representation of its double cover $S p(1)$. 
Proposition 3.3.8. We have an isomorhism of $S p(1)$-representations

$$
\operatorname{Hom}_{\mathbb{R}}(U, V) \cong \mathbb{R}^{3} \otimes_{\mathbb{R}} \operatorname{Hom}_{\mathbb{H}}(\tilde{U}, \tilde{V}) \oplus \operatorname{Hom}_{\mathbb{H}}(\tilde{U}, \tilde{V}) .
$$

Moreover,

$$
B_{+}=\mathbb{R}^{3} \otimes \operatorname{Hom}_{\mathbb{H}}(\tilde{U}, \tilde{V}), \quad B_{-}=\operatorname{Hom}_{\mathbb{H}}(\tilde{U}, \tilde{V})
$$

Proof. First observe that

$$
\operatorname{Hom}_{\mathbb{R}}(U, V)_{\mathbb{C}} \cong\left(U^{*} \otimes_{\mathbb{R}} V\right)_{\mathbb{C}} \cong U_{\mathbb{C}} \otimes_{\mathbb{C}} V_{\mathbb{C}}
$$

Further, it follows from Proposition 3.1.1 that

$$
U_{\mathbb{C}} \otimes_{\mathbb{C}} V_{\mathbb{C}} \cong W \otimes_{\mathbb{C}} \tilde{U} \otimes_{\mathbb{C}} W \otimes_{\mathbb{C}} \tilde{V} \cong W \otimes_{\mathbb{C}} W \otimes_{\mathbb{C}}\left(\tilde{U} \otimes_{\mathbb{C}} \tilde{V}\right)
$$

But according to the Clebsch-Gordon formula [12] we have $W \otimes_{\mathbb{C}} W \cong W_{2} \oplus \mathbb{C}$ and therefore

$$
\operatorname{Hom}_{\mathbb{R}}(U, V)_{\mathbb{C}} \cong\left(W_{2} \oplus \mathbb{C}\right) \otimes_{\mathbb{C}} H_{\mathrm{Com}}\left(\tilde{U} \otimes_{\mathbb{C}} \tilde{V}\right),
$$

where $H_{0} m_{\mathbb{C}}\left(\tilde{U} \otimes_{\mathbb{C}} \tilde{V}\right)$ denotes the space of operators $A$ such that $A J_{1}=I_{1} A$. Since the real part of $H_{0} m_{\mathbb{C}}\left(\tilde{U} \otimes_{\mathbb{C}} \tilde{V}\right)$ is the set of fixed points of the operator $A \mapsto-I_{2} A J_{2}$, i.e $\operatorname{Hom}_{\mathbb{H}}\left(\tilde{U} \otimes_{\mathbb{C}} \tilde{V}\right)$, we get

$$
\begin{aligned}
\operatorname{Hom}_{\mathbb{R}}(U, V) & \cong\left(\left[W_{2}\right]_{r} \oplus \mathbb{R}\right) \otimes_{\mathbb{R}}\left[\operatorname{Hom}_{\mathbb{C}}\left(\tilde{U} \otimes_{\mathbb{C}} \tilde{V}\right)\right]_{r} \cong \\
& \cong\left[W_{2}\right]_{r} \otimes_{\mathbb{R}} H_{\mathbb{H}}(\tilde{U}, \tilde{V}) \oplus \operatorname{Hom}_{\mathbb{H}}(\tilde{U}, \tilde{V}) .
\end{aligned}
$$

Now it only remains to note that $\left[W_{2}\right]_{r} \cong \mathbb{R}^{3}$.

\subsubsection{Aholomorphic maps between hyperKähler mani- folds}

Let $\left(X, J_{1}, J_{2}, J_{3}\right)$ and $\left(M, I_{1}, I_{2}, I_{3}\right)$ be two hyperKähler manifolds (in this section one may work equally well with hypercomplex manifolds, but we will remain in the Riemannian category) and let $u: X \rightarrow M$ be a smooth map. Then its differential $u_{*}$ is a linear map between the two quaternionic modules $T X$ and $T M$.

Definition 3.3.9 (Aholomorphic map). A map $u: X \rightarrow M$ is said to be aholomorphic if its differential is aquaternionic, i.e if

$$
C\left(u_{*}\right)=I_{1} u_{*} J_{1}+I_{2} u_{*} J_{2}+I_{3} u_{*} J_{3}=u_{*} .
$$


Proposition 3.3.10. A map $u$ is aholomorphic if and only if the triholomorphic (i.e. quaternionic linear) component of its differential $u_{*}$ vanishes.

Proof. This is a consequence of the corresponding statement for linear operators (see Proposition 3.3.6).

Aholomorphic maps were already studied, however under a variety of different names. They naturally arise in the supersymmetric gauged $\sigma$-model and appeared in physical literature [2, 25] for the first time as "triholomorphic maps" (warning: we reserve the term "triholomorphic" for quaternionic linear maps) or "hyperinstantons". Such maps were studied also from a mathematical viewpoint by Chen [15], Chen and Li [16] ("quaternionic maps"), Wang [71] ("triholomorphic maps"). Joyce [42] ("q-holomorphic functions") considered the case of a flat target manifold $\mathbb{H}$. Equation (3.15) was known long ago and was introduced in 1934 by Fueter [28]("regular functions") for the simplest case of maps $u: \mathbb{H} \rightarrow \mathbb{H}$ in his attempts to construct a quaternionic analogue of the theory of complex holomorphic maps. The equation (3.15) is often called the Cauchy-Riemann-Fueter equation. An extensive exposition of the theory can be found in 64.

In the author's opinion the proposed term aholomorphic map better reflects the properties of maps satisfying equation (3.15), namely the fact that the differential of solutions to (3.15) has a vanishing triholomorphic component.

Observe that the aholomorphicity condition (3.15) is elliptic only in case when a source manifold $X$ is four-dimensional.

In the new terminology we have the following proposition.

Proposition 3.3.11. Assume that $X^{4}$ is hyperKähler. Then a spinor $u$ : $X \rightarrow M$ is harmonic if and only if it is aholomorphic.

\subsubsection{Aholomorphic sections}

Definition 3.3.12. Let $E \longrightarrow X$ be a vector bundle. A rank 3 subbundle $\mathcal{I} \subset \operatorname{End}(E)$ is said to be a quaternionic structure, if it has local bases $\left(I_{1}, I_{2}, I_{3}\right)$ such that $I_{l}^{2}=-I d ; I_{1} I_{2}=-I_{2} I_{1}=I_{3}$.

Note that the quaternionic structure $\mathcal{I}$ considered as a vector bundle has a natural euclidean metric and orientation.

Now suppose that $E_{1}$ and $E_{2}$ are vector bundles with quaternionic structures $\mathcal{I}_{1}$ and $\mathcal{I}_{2}$. Futhermore, suppose that $\mathcal{I}_{1}$ and $\mathcal{I}_{2}$ are isomorphic (as oriented euclidean vector bundles) and fix an isomorphism $\mathrm{F}$ between them. 
The isomorphism $\mathrm{F}$ defines an operator $C=C_{\mathrm{F}} \in \Gamma\left(\operatorname{End}\left(\operatorname{Hom}\left(E_{1}, E_{2}\right)\right)\right)$ in a natural way. Indeed, pick a local basis $\left(I_{1}, I_{2}, I_{3}\right)$ of $\mathcal{I}_{1}$ and denote by $\left(J_{1}, J_{2}, J_{3}\right)$ its image under F. Then

$$
C=J_{1} \cdot I_{1}+J_{2} \cdot I_{2}+J_{3} \cdot I_{3}
$$

and it does not depend on the choice of a local basis.

Definition 3.3.13. A section $A \in \Gamma\left(\operatorname{Hom}\left(E_{1}, E_{2}\right)\right)$ is said to be aquaternionic with respect to the isomorphism $\mathrm{F}$, if $C_{\mathrm{F}}(A)=A$.

Definition 3.3.14. Let $f$ be a map between two quaternionic Kähler manifolds $N_{1}$ and $N_{2}$. Assume that $f^{*} \mathcal{I}_{2}$ is isomorphic to $\mathcal{I}_{1}$ and fix an isomorphism $\mathrm{F}: \mathcal{I}_{1} \longrightarrow f^{*} \mathcal{I}_{2}$. We say that the map $f$ is aholomorphic with respect to $\mathrm{F}$ if $d f \in \Gamma\left(T^{*} N_{1} \otimes f^{*} T N_{2}\right)$ is aquaternionic.

Observe that a map $f$ between two hyperKähler manifolds is aholomorhic in the sense of Definition 3.3 .9 if it is aholomorhic with respect to the identity isomorphism.

Aholomorphic maps between quaternionic Kähler manifolds were studied mainly in the realm of topological $\sigma$-model [33, 2] although under different names.

Pick a $G$-principal bundle $\pi^{G}: P_{G} \rightarrow X$ with a connection $a \in \Omega^{1}\left(P_{G} ; \mathfrak{g}\right)$. Let $N$ be an (almost) quaternionic Kähler manifold with a quaternionic Kähler action of $G$. Consider an equivariant map $\varphi \in \operatorname{Map}^{G}\left(P_{G}, N\right)$ or equivalently a corresponding section $\varphi \in \Gamma(\mathcal{N})$, where $\mathcal{N}=P_{G} \times_{G} N$. Observe, that

$$
\nabla^{a} \varphi \in \Gamma\left(T^{*} X \otimes \pi_{!}^{G}\left(\varphi^{*} T N\right)\right) \cong \Gamma\left(\operatorname{Hom}\left(T X, \pi_{!}^{G}\left(\varphi^{*} T N\right)\right)\right)
$$

and both vector bundles $T X$ and $\pi_{!}^{G}\left(\phi^{*} T N\right)$ have quaternionic Kähler structures. Indeed, since the action of $G$ is quaternionic Kähler, the latter bundle has the induced quaternionic structure from $T N$. But the tangent bundle of any oriented Riemannian 4-dimensional manifold automatically carries an almost quaternionic structure. In fact there are two such non-isomorphic structures: one can be identified with $\Lambda_{+}^{2} X$ and the other with $\Lambda_{-}^{2} X$. We prefer $\Lambda_{+}^{2} X$ since it better fits our conventions.

Now assume that $\Lambda_{+}^{2} X$ and $\pi_{!}^{G}\left(\varphi^{*} \mathcal{I}\right)$ are isomorphic and fix an isomorphism $\mathrm{F}: \Lambda_{+}^{2} X \stackrel{\sim}{\longrightarrow} \pi_{!}^{G}\left(\varphi^{*} \mathcal{I}\right)$, where $\mathcal{I}$ is the structure bundle of $N$.

Definition 3.3.15. We say that a section $\varphi \in \Gamma(\mathcal{N})$ is aholomorphic with respect to the pair $(a, \mathrm{~F})$, if its covariant derivative $\nabla^{a} \varphi$ is an aquaternionic section of $\operatorname{Hom}\left(T X, \pi_{!}^{G}\left(\varphi^{*} T N\right)\right)$. 
Remark 3.3.16. Locally the notion of aholomorphicity can be expressed as follows. Pick an orthonormal oriented local basis $\mathrm{v}=\left(\mathrm{v}_{0}, \mathrm{v}_{1}, \mathrm{v}_{2}, \mathrm{v}_{3}\right)$ of $T X$ and consider $\varphi$ as an equivariant map. Further, $v$ determines a local basis of $\Lambda_{+}^{2} T X$. The isomorphism $\mathrm{F}$ in turn determines a local basis $\left(I_{1}, I_{2}, I_{3}\right)$ of the pull-back of $\mathcal{I}$. Then $\varphi$ is aholomorphic iff

$$
\varphi_{*} \tilde{\mathrm{V}}_{0}+I_{1} \varphi_{*} \tilde{\mathrm{V}}_{1}+I_{2} \varphi_{*} \tilde{\mathrm{V}}_{2}+I_{3} \varphi_{*} \tilde{\mathrm{V}}_{3}=0
$$

where $\tilde{\mathrm{v}}_{l}$ denotes the horizontal lift of $\mathrm{v}_{l}$ with respect to the connection $a$.

\subsection{Harmonic spinors and aholomorphic sec- tions}

In this section we assume that the source manifold $X$ is spinor. This is not essential, however the exposition is clearer.

Denote by $P_{\text {Spin }}$ the $\operatorname{Spin}(4)$-structure of $X, P_{+}=P_{\text {Spin }} / S p_{-}(1)$ is then an $S p_{+}(1)$-structure. Since the subgroup $S p_{-}(1)$ acts trivially on $M$ we may assume that spinors $u$ are elements of $\operatorname{Map}^{S p_{+}(1)}\left(P_{+}, M\right)$. Suppose also that the permuting action of $S p(1)$ on $M$ can be extended to the permuting action of $\mathbb{H}^{*}$. Then $N=M / \mathbb{H}^{*}$ is a quaternionic Kähler manifold and we have a natural projection $\kappa: M \rightarrow N$.

As it was already pointed out in Chapter 2 the manifold $M$ can be recovered by $N$. More precisely, denote by $\mathcal{F} \longrightarrow N$ an $S O(3)$-principal bundle of local almost complex structures on $N$. Take $W_{0}$ as in Example 3.2 .8 and consider a fibre bundle $\mathcal{F} \times{ }_{S O(3)} W_{0}$ where the action is given via quaternionic multiplication (by quaternionic conjugate) on the right. It turns out that the total space of this bundle is the original manifold $M$ (see [65] for details). Moreover, the permuting action of $S p(1)$ now can be described as a fiberwise multiplication on the left.

Remark 3.4.1. There is an inclusion $\mathcal{F} \hookrightarrow M$. Indeed, from the standard inclusion $S^{3} \hookrightarrow \mathbb{H}$ one obtaines $S O(3) \hookrightarrow W_{0}$ and therefore

$$
\mathcal{F} \cong \mathcal{F} \times_{S O(3)} S O(3) \hookrightarrow \mathcal{F} \times_{S O(3)} W_{0}=M
$$

Note also that this inclusion can be written explicitly as $f \mapsto[f, 1]$ so that $f \cdot q \mapsto[f \cdot q, 1]=\bar{q}[f, 1]$. This implies in particular that an invariant map $P_{+} \longrightarrow \mathcal{F}$ becomes equivariant if we consider it as a map $P_{+} \longrightarrow M$. Vice versa, if one takes the composition of an equivariant map with a projection $M \longrightarrow M / \mathbb{R}^{*} \cong \mathcal{F}$ then it becomes invariant. 
Let $u \in \operatorname{Map}^{S p_{+}(1)}\left(P_{+}, M\right)$ be a spinor. Then we have the following commutative diagram

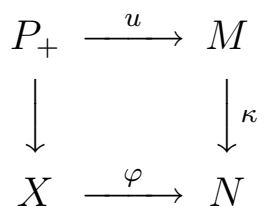

where $\varphi$ is the projection of $u$. It means that one can consider a spinor $u$ as a pair $(\varphi, \psi)$ with an equivariant map $\psi: P_{+} \longrightarrow \varphi^{*} M$. It follows from the previous remark that the map $\psi$ gives an invariant isomorphism of principal bundles $\Psi: P_{\Lambda_{+}^{2}} \longrightarrow \mathcal{F}$, where $P_{\Lambda_{+}^{2}}$ is the principal bundle of $\Lambda_{+}^{2} X$ or equivalently $P_{+} /\{ \pm 1\}$.

We can describe $\psi$ equivalently as follows. First, consider the $S_{+}(3) \times$ $S O(3)$-principle bundle $P_{\Lambda_{+}^{2}} \times \varphi^{*} \mathcal{F}$ over $X$. Let $\widetilde{W}_{0}^{+}$denote the manifold $\mathbb{H}^{*} / \pm 1$ together with the following action of $S O(3) \times S O(3)$ :

$$
\left(\left(\left[q_{1}\right],\left[q_{2}\right]\right),[h]\right) \mapsto\left[q_{1} h \bar{q}_{2}\right] .
$$

Then we obtain a fibre bundle $\widetilde{\mathcal{W}}_{0}^{+}=\left(P_{\Lambda_{+}^{2}} \times \varphi^{*} \mathcal{F}\right) \times_{S O_{+}(3) \times S O(3)} \widetilde{W}_{0}^{+}$over $X$. One easily sees from the construction that $\widetilde{\mathcal{W}}_{0}^{+}=\left(P_{\Lambda_{+}^{2}} \times_{S O_{+}(3)} \varphi^{*} M\right)$. Thus $\psi$ is a section of $\widetilde{\mathcal{W}}_{0}^{+}$.

Theorem 3.4.2. Under the above conventions, harmonic spinors $u$ are in bijective correspondence with pairs $(\varphi, \psi)$ such that $\varphi$ is an aholomorphic map with respect to $\Psi$ and $\psi$ is a harmonic spinor with respect to to the Dirac operator with coefficients (as described in remark 3.2.13 b).

Proof. If the action of $S p(1)$ on $M$ extends to the action of $\mathbb{H}^{*}$, then [65] the vertical bundle $\mathcal{V}_{M}$ of $\kappa: M \rightarrow N$ is $\operatorname{span}_{\mathbb{R}}\left(Y_{0}, I_{1} Y_{0}, I_{2} Y_{0}, I_{3} Y_{0}\right)=\operatorname{span}_{\mathbb{H}}\left(Y_{0}\right)$, where the vector field $Y_{0}$ is a Killing vector field of $\mathbb{R}^{*} \subset \mathbb{H}^{*}$. In particular, $\mathcal{V}_{M}$ is invariant with respect to complex structures of $M$. Define a horizontal distribution $\mathcal{H}_{M}$ as an orthogonal complement to the vertical subbundle:

$$
\mathcal{H}_{M}=\mathcal{V}_{M}^{\perp} \subset T M \text {. }
$$

Since complex structures of $M$ are orthogonal operators, they also preserve the horizontal distribution $\mathcal{H}_{M}$.

Now take $\mathrm{v}_{l}$ 's and $\mathrm{w}_{l}$ 's as in Lemma 3.2.15 so that equation (3.11) holds. Further, decompose $\mathrm{w}_{l}=u_{*} \mathrm{v}_{l}$ into horizontal and vertical components: $\mathrm{w}_{l}=$

\footnotetext{
${ }^{\mathrm{b}}$ in this case $G=S O(3), P_{G}=\varphi^{*} \mathcal{F}$ equipped with the pull-back of the natural connection of $\mathcal{F}$
} 
$\mathrm{w}_{l}^{h}+\mathrm{w}_{l}^{v}$. Then equation 3.11 splits:

$$
\begin{gathered}
\mathrm{w}_{0}^{h}+I_{1} \mathrm{w}_{1}^{h}+I_{2} \mathrm{w}_{2}^{h}+I_{3} \mathrm{w}_{3}^{h}=0, \\
\mathrm{w}_{0}^{v}+I_{1} \mathrm{w}_{1}^{v}+I_{2} \mathrm{w}_{2}^{v}+I_{3} \mathrm{w}_{3}^{v}=0 .
\end{gathered}
$$

But equation (3.16) is equivalent to the aholomorphicity of $\varphi$ with respect to $\Psi$. On the other hand, equation (3.17) is equivalent to the requirement that the spinor $\psi \in \Gamma\left(\tilde{\mathcal{W}}_{0}^{+}\right)$is harmonic with respect to the twisted $S O$-Dirac operator.

Remark 3.4.3. In general, $\varphi$ and $\psi$ can not be defined separately, since aholomorphicity implies the knowledge of $\psi$, and $\psi$ itself implies $\varphi$ by its very definition. However, when $N$ is itself a hyperKähler manifold, then $M$ is the trivial bundle: $M=N \times \mathbb{H}^{*}$. In this case $\varphi$ and $\psi$ become independent. One easily sees that $\psi \in \Gamma\left(W^{+}\right)$is just the usual (linear) spinor. 


\section{Chapter 4}

\section{Generalized Seiberg-Witten Equations over Kähler Surfaces and Holomorphic Curves}

In this chapter a generalization of the Seiberg-Witten equations based on the nonlinear Dirac operator is studied. The definition appeared in [59] (see also [69]). Suppose that the source 4-manifold $X$ is Kähler; then we show in Theorem 4.2 .3 that solutions to the generalized Seiberg-Witten equations have a holomorphic description similarly to the classical case (see Theorem 1.1.8). This theorem also allows us to obtain connection between the Seiberg-Witten theory and other gauge theories [40, 58, 18, which appeared in a slightly different context, mainly in symplectic topology. In Section 4.5 we study the THC-system defined in [40] over the product of two holomorphic curves. Although interesting on its own, this section mainly serves for us as a prototype of the Seiberg-Witten equations over the product of two holomorphic curves, which is the subject of Section 4.6. We show (modulo the adiabatic limit conjecture), that there is a relation between the THC-system, holomorphic curves as in the Gromov theory and symplectic vortex equations [18]. Analogous results are obtained for the generalized Seiberg-Witten equations in Theorem 4.6.3,

The connection between holomorphic curves and classical Seiberg-Witten equations was studied in [17]. Such connection was discovered for the first time by Taubes [68], however his approach is different. 


\subsection{Generalized Seiberg-Witten equations}

Let $P_{\text {Spin }^{c}} \rightarrow X$ be a $\operatorname{Spin}^{c}(4)$ principal bundle with connection $\hat{a}$ determined by a connection $a$ on the determinant bundle $P_{d e t}=P_{\text {Spin }^{c}} / \operatorname{Spin}(4)$ and the Levi-Civita one on $P_{s o}$. We also fix a hyperKähler manifold $M$ with the action of $U(2)$ as described in Section 3.2. Recall, that maps $u \in \operatorname{Map}^{\operatorname{Spin}^{c}(4)}\left(P_{\text {Spin }^{c}}, M\right) \cong \operatorname{Map}^{U_{+}(2)}\left(P_{+}, M\right)$ play a role of spinors, where $P_{+}=P_{\text {Spinc }} / S p_{-}(1)$.

The triholomorphic action of the center of $U(2)$ gives rise to a momentum map

$$
\mu: M \longrightarrow \mathbb{R}^{3} \cong \operatorname{Im} \mathbb{H} .
$$

The requirement that the action of $S p(1)$ is permuting gives that the target $\mathbb{R}^{3}$ of the momentum map is in fact the standard representation of $S p(1) / \pm 1 \cong$ $S O(3)$. Hence the composition of a spinor $u \in \operatorname{Map}^{\operatorname{Spin}^{c}(4)}\left(P_{\text {Spin }^{c}}, M\right)$ with the momentum map $\mu$ is an equivariant map

$$
\mu \circ u: P_{\text {Spin }^{c}} \longrightarrow \mathbb{R}_{+}^{3},
$$

where as before we use the subscript + to keep in mind that the action is induced by the $S O_{+}(3)$. It follows that any spinor $u$ generates a self-dual 2-form $\Phi(u)$.

Definition 4.1.1. The first order differential equations

$$
\left\{\begin{array}{l}
\mathcal{D}_{a} u=0, \\
F_{a}^{+}+\Phi(u)=0 .
\end{array}\right.
$$

for a spinor $u \in \operatorname{Map}^{\operatorname{Spin}^{c}(4)}\left(P_{\text {Spin }^{c}}, M\right)$ and a connection $a \in \mathcal{A}\left(P_{\text {det }}\right)$ are called (generalized) Seiberg-Witten equations.

Remark 4.1.2. The above definition was given by Pidstrygach [59]. The analogous setting for 3-manifolds was described by Taubes [69].

\subsection{Generalized Seiberg-Witten Equations over Kähler Surfaces}

In this section we will consider the generalized Seieberg-Witten equations in assumption that the source manifold $X^{4}$ carries a Kähler structure. In this case the principal $S O(3)$ bundle $P_{\Lambda_{+}^{2}}$ reduces to an $S^{1}$ principal bundle (we prefer to denote this group by $S_{r}^{1}$ ) so that we have the reduction of $P_{+}=P_{U(2)_{+}}$to the $H=\left(S_{r}^{1} \times S_{0}^{1}\right) / \pm 1 \cong \mathbb{T}^{2}$ principal bundle $P_{\text {red }}$.

We start with the following auxiliary lemma. 
Lemma 4.2.1. Let $G^{\prime}$ be a Lie subgroup of a Lie group $G$ and $P^{\prime}$ be a principal $G^{\prime}$-bundle over $X$. Define a principal $G$-bundle $P_{G}$ as $P^{\prime} \times_{G}$, G. Assume also that the group $G$ acts on a manifold $M$. Then one has a canonical isomorphism

$$
\operatorname{Map}^{G}\left(P_{G}, M\right) \cong \operatorname{Map}^{G^{\prime}}\left(P^{\prime}, M\right) .
$$

Proof. Pick an equivariant map $u: P^{\prime} \rightarrow M$. Then the map $\tilde{u}:[p, g] \mapsto$ $g^{-1} u(p)$ belongs to $\operatorname{Map}^{G}\left(P_{\mathrm{G}}, M\right)$. On the other hand the map $p \mapsto[p, \mathbf{1}]$ provides an embedding of $P^{\prime}$ into $P_{\mathrm{G}}$ and therefore we have an inclusion $\operatorname{Map}^{G}\left(P_{\mathrm{G}}, M\right) \subset \operatorname{Map}^{G^{\prime}}\left(P^{\prime}, M\right)$.

Corollary 4.2.2. If $X$ is a Kähler surface, then

$$
\operatorname{Map}^{U(2)}\left(P_{+}, M\right) \cong \operatorname{Map}^{H}\left(P_{r e d}, M\right) .
$$

Proof. The statement follows from the previous lemma, since we have an embedding $H \hookrightarrow\left(S p(1) \times S_{0}^{1}\right) / \pm 1 \cong U(2)$.

Theorem 4.2.3. Suppose the source 4-manifold $X$ is a Kähler surface. Then a pair $(a, u) \in \mathcal{A}\left(P_{\text {det }}\right) \times \operatorname{Map}^{H}\left(P_{\text {red }}, M\right)$ is a solution of the Seiberg-Witten equations if and only if the following holds:

1. a is a holomorphic structure, i.e. $\left(F_{a}\right)^{(0,2)}=0$,

2. $\mu_{c} \circ u \equiv 0, \quad \mu_{c}=\mu_{2}+\mu_{3} i: M \rightarrow \mathbb{C}$,

3. $F_{a}^{+}+\left(\mu_{1} \circ u\right) \omega_{X}=0$,

4. $u: P_{\text {red }} \rightarrow M$ is a holomorphic equivariant map ${ }^{\text {a }}$ with respect to the connection $\hat{a}$, where the complex structure on $M$ is given by $-I_{1}$ and $\hat{a}$ is generated by a and the Levi-Civita connection.

Proof. Obviously, any solution of the Seiberg-Witten equations is an absolute minimum of the functional

$$
S(a, u)=\frac{1}{2}\left\|F_{a}^{+}+\Phi(u)\right\|^{2}+\left\|\mathcal{D}_{a} u\right\|^{2} .
$$

Using the Weitzenböck formula, one can rewrite the functional $S$ as follows [59]:

$$
S(a, u)=\frac{1}{2}\left\|F_{a}^{+}\right\|^{2}+\frac{1}{2}\|\Phi(u)\|^{2}+\left\|\nabla^{\hat{a}} u\right\|^{2}+\int_{X} \rho(u) \frac{s}{4} d v o l_{X},
$$

${ }^{\mathrm{a}}$ see Convention 3.2 .19 
where $\rho: M \rightarrow \mathbb{R}$ is the hyperKähler potential and $s$ is the scalar curvature of $X$.

Now fix $z_{r} \in S_{r}^{1} \subset \mathbb{H}^{*}$ and consider $u^{\prime}=z_{r} \cdot u=L_{z_{r}} u, L_{z_{r}}: M \rightarrow M$. Since $S\left(a, u^{\prime}\right)=S(a, u)$ it follows that $\left(a, u^{\prime}\right)$ is a solution of the SeibergWitten equations too.

On the other hand, solutions to the Seiberg-Witten equations are also minima of the functional

$$
S_{1}(a, u)=\left\|F_{a}^{+}+\Phi(u)\right\|^{2}+\left\|\mathcal{D}_{a} u\right\|^{2} .
$$

Applying the Weitzenböck formula again, one gets

$$
S_{1}(a, u)=\left\langle F_{a}^{+}, \Phi(u)\right\rangle+\left\|F_{a}^{+}\right\|^{2}+\|\Phi(u)\|^{2}+\left\|\nabla^{\hat{a}} u\right\|^{2}+\int_{X} \rho(u) \frac{s}{4} d v o l_{X} .
$$

Since the source manifold $X$ is Kähler we have [20] the decomposition

$$
\Omega_{+}^{2}(X) \cong \Omega^{2,0}(X) \oplus \Omega^{0}(X) \omega_{X} \oplus \Omega^{0,2}(X),
$$

and therefore we may write $F_{a}^{+}=\left(F_{a}^{+}\right)^{1,1}+\mathcal{F}$, where the last summand belongs to $\Omega^{2,0} \oplus \Omega^{0,2}$. Similarly, we may decompose $\Phi(u)=(\Phi(u))^{1,1}+$ $\Phi_{c}(u)=\left(\mu_{1} \circ u\right) \omega_{X}+\Phi_{c}(u)$, where $\Phi_{c}(u)$ is the $\Omega^{2,0} \oplus \Omega^{0,2}$ component of $\Phi(u)$. Observe that the 2 -form $\Phi_{c}(u)$ is real and can be identified with the equivariant map $\mu_{c} \circ u$. Then

$$
\begin{aligned}
S_{1}(a, u)=\mu_{1} \circ u\left\langle\left(F_{a}^{+}\right)^{1,1}\right. & \left., \omega_{X}\right\rangle+\left\langle\mathcal{F}, \Phi_{c}(u)\right\rangle+ \\
& +\left\|F_{a}^{+}\right\|^{2}+\|\Phi(u)\|^{2}+\left\|\nabla^{\hat{a}} u\right\|^{2}+\int_{X} \rho(u) \frac{s}{4} d v^{2} l_{X} .
\end{aligned}
$$

Now all the summands on the right-hand side of the above formula remain invariant if we replace $u$ by $u^{\prime}$ with the only exception: the term $\left\langle\mathcal{F}, \Phi_{c}(u)\right\rangle$ is multiplied by $z_{r}^{2}$. It follows, that the term must vanish. Since $F_{a}^{+}=-\Phi(u)$, we get $\mathcal{F}=-\Phi_{c}(u)$ and consequently $\mathcal{F}=0, \Phi_{c}(u)=0$. Thus, for any solution of the Seiberg-Witten equations statements 1-3 of the theorem hold.

Observe that on the other hand, the second equation of (4.1) follows from the statements $1-3$. Indeed, since $a$ is a unitary connection, $F_{a}^{(0,2)}=0$ implies [20] $F_{a}^{(2,0)}=0$. It remains to recall the decomposition 4.2].

The complex structure of $X$ (or, equivalently, the Kähler form) provides a reduction of $P_{+}$to $P_{\text {red }}$. It means that we have a natural embedding $P_{\text {red }} \subset P_{+}$ such that the horizontal subbundle of $T P_{\text {red }}$ coincides with the pull-back of the horizontal subbundle of $T P_{+}$. We do not distinguish between these two horizontal subbundles in notations. 
Let $u$ be as in the statement of the theorem. According to Corollary 4.2.2 we have a natural isomorphism $\operatorname{Map}^{H}\left(P_{\text {red }}, M\right) \cong \operatorname{Map}^{U(2)}\left(P_{+}, M\right)$. Denote by $\tilde{u}$ the image of $u$ in $\operatorname{Map}^{U(2)}\left(P_{+}, M\right)$. By Corollary 3.2.16, the spinor $\tilde{u}$ satisfies $\tilde{u}_{*}-I_{1} \tilde{u}_{*} J_{1}-I_{2} \tilde{u}_{*} J_{2}-I_{3} \tilde{u}_{*} J_{3}=0$ on $\mathcal{H} \rightarrow P_{+}$. It follows from the above observation that it is equivalent to $u_{*}-I_{1} u_{*} J_{1}-I_{2} u_{*} J_{2}-I_{3} u_{*} J_{3}=0$ on $\mathcal{H} \rightarrow P_{\text {red }}$. Since the reduction of $P_{+}$to $P_{\text {red }}$ is provided by the complex structure of $X$, we may assume that $J_{1}$ is its pull-back to $\mathcal{H}$.

Pick an arbitrary vector $\mathrm{v} \in T . X$ and denote $\mathrm{w}_{1}=u_{*} \hat{\mathrm{v}}-I_{1} u_{*} J_{1} \hat{\mathrm{v}}, \mathrm{w}_{2}=$ $u_{*} J_{2} \hat{\mathrm{v}}, \mathrm{w}_{3}=u_{*} J_{3} \hat{\mathrm{v}}$. Then

$$
\mathrm{w}_{1}-I_{2} \mathrm{w}_{2}-I_{3} \mathrm{w}_{3}=0 .
$$

Since the action of $S_{r}^{1}$ rotates $I_{2}$ and $I_{3}$, one has

$$
\begin{aligned}
& L_{z_{r}} I_{2} L_{\bar{z}_{r}}=\alpha I_{2}-\beta I_{3}, \\
& L_{z_{r}} I_{3} L_{\bar{z}_{r}}=\beta I_{2}+\alpha I_{3},
\end{aligned}
$$

where $z_{r}^{2}=\alpha+\beta i$. Then applying $L_{z_{r}}$ to equation 4.3 one has:

$$
L_{z_{r}} \mathrm{w}_{1}-\left(\alpha I_{2}-\beta I_{3}\right) L_{z_{r}} \mathrm{w}_{2}-\left(\beta I_{2}+\alpha I_{3}\right) L_{z_{r}} \mathrm{w}_{3}=0 .
$$

On the other hand the harmonicity of $u^{\prime}$ implies

$$
L_{z_{r}} \mathrm{w}_{1}-I_{2} L_{z_{r}} \mathrm{w}_{2}-I_{3} L_{z_{r}} \mathrm{w}_{3}=0 .
$$

Subtracting the above two equations one obtains that the equality

$$
\left(\alpha I_{2}-\beta I_{3}\right) L_{z_{r}} \mathrm{w}_{2}+\left(\beta I_{2}+\alpha I_{3}\right) L_{z_{r}} \mathrm{w}_{3}=I_{2} L_{z_{r}} \mathrm{w}_{2}+I_{3} L_{z_{r}} \mathrm{w}_{3}
$$

holds for all $\alpha, \beta$ such that $\alpha^{2}+\beta^{2}=1$.

Now put $\alpha=0, \beta=1$. Then equation (4.4) gives $I_{3} L_{z_{r}}\left(\mathrm{w}_{2}-\mathrm{w}_{3}\right)=$ $I_{2} L_{z_{r}}\left(\mathrm{w}_{2}-\mathrm{w}_{3}\right)$ which in turn implies $\mathrm{w}_{2}=\mathrm{w}_{3}=\mathrm{w}$. Thus we can rewrite equation (4.4) as $\left((\alpha+\beta) I_{2}+(\alpha-\beta) I_{3}\right) L_{z_{r}} \mathrm{w}=\left(I_{2}+I_{3}\right) L_{z_{r}} \mathrm{w}$ and this clearly implies $\mathrm{w}=0$. Since $\mathrm{v}$ was arbitrary we see that $u$ satisfies

$$
u_{*}-I_{1} u_{*} J_{1}=0,
$$

i.e. $u$ is an equivariant holomorphic map if one considers $M$ as a complex manifold with the complex structure $-I_{1}$.

On the other hand, if $u$ is a holomorphic equivariant map, i.e. if (4.5) holds, then one gets $I_{2} u_{*} J_{2}+I_{3} u_{*} J_{3}=0$. It follows that $u$ also satisfies $u_{*}-I_{1} u_{*} J_{1}-I_{2} u_{*} J_{2}-I_{3} u_{*} J_{3}=0$, i.e. $u$ is a harmonic spinor. 
Remark 4.2.4. As we have already remarked, under the assumption that the source manifold $X$ is Kähler, the group $U(2)_{+}$reduces to $H=\left(S_{r}^{1} \times\right.$ $\left.S_{0}^{1}\right) / \pm 1$. Consequently, it is possible to define Seiberg-Witten type equations on 4-dimensional Kähler manifolds when the target hyperKähler manifold $M$ instead of $\mathbb{H}^{*}$ (or $S p(1)$ ) permuting action admits only an action of $H$, where the component $S_{0}^{1}$ acts triholomorphically and $S_{r}^{1}$ fixes $I_{1}$ while rotating $I_{2}$ and $I_{3}$. Such definition belongs to $\mathrm{V}$. Pidstrygach and equations of such type were first considered in R. Waldmüller diploma thesis [70]. Although our proof of the first three statements uses the fact that $M$ admits a permuting $\mathbb{H}^{*}$ action, however they still remain true. The proof of the forth one is also valid when $M$ admits only an $S_{r}^{1}$-action.

It is straightforward to check that the map $\mu_{c}: M \rightarrow \mathbb{C}, \mu_{c}=\mu_{2}+\mu_{3} i$ is $I_{1}$-holomorphic and therefore

$$
M_{c}=\left\{m \in M \mid \mu_{c}(m)=0\right\}
$$

is an $I_{1}$-complex submanifold (assuming that it is submanifold) of $\left(M, I_{1}\right)$. Consequently, $\left(M_{c},-I_{1}\right)$ is also a complex submanifold of $\left(M,-I_{1}\right)$. Moreover, both $S_{r}^{1}$ and $S_{0}^{1}$ act holomorphically on $M_{c}$.

With this in mind, Theorem 4.2.3 may be interpreted in the following way. Recall that we have an $H$-principal bundle $P_{\text {red }}$ over a Kähler surface $X$ with two projections: $P_{r e d} \rightarrow P_{\text {red }} / S_{r}^{1}=P_{\text {det }}$ and $P_{\text {red }} \rightarrow P_{\text {red }} / S_{0}^{1}=P_{r}$, where $P_{r}$ is a principal bundle of the anticanonical line bundle of $X$. A choice of connection $a$ on $P_{\text {det }}$ uniquely defines a connection $\hat{a}$ on $P_{\text {red }}$ by the requirement that the connection on $P_{r}$ is induced by the Levi-Civita connection. Next, we may regard nonlinear spinors $u$ as equivariant maps $P_{\text {red }} \rightarrow M_{c}$. Now the first statement of the theorem says that $\hat{a}$ equips $P_{\text {red }}$ with a holomorphic structure; then the fourth one says that $u$ is a holomorphic equivariant map, i.e. $\bar{\partial}_{\hat{a}} u=0$.

Further, denote by $\Lambda: \Omega^{p+1, q+1}(X) \rightarrow \Omega^{p, q}(X)$ the adjoint operator of $\mathrm{L}: \Omega^{p, q}(X) \rightarrow \Omega^{p+1, q+1}(X), \alpha \mapsto \alpha \wedge \omega_{X}$. It follows from the decomposition (4.2) that $\Lambda F_{a}^{+}=\Lambda F_{a}$. Now since $a$ is a $U(1)$ connection with $F_{a}^{(0,2)}=0$ we have also $F_{a}^{(2,0)}=0$. Therefore, for a unitary connection $a$ such that $F_{a}^{(0,2)}=0$ we may equivalently rewrite the equation $F_{a}^{+}+\left(\mu_{1} \circ u\right) \omega_{X}=0$ as $\Lambda F_{a}+\mu_{1} \circ u=0$. Summing up we get the following corollary.

Corollary 4.2.5. Let $X$ be a Kähler surface. Then there is a one-to-one correspondence between solutions of the Seiberg-Witten equations (4.1) and the following system

$$
\left\{\begin{array}{l}
\bar{\partial}_{\hat{a}} u=0, \\
\Lambda F_{a}+\mu_{1} \circ u=0,
\end{array}\right.
$$


where $u \in \operatorname{Map}^{H}\left(P_{\text {red }}, M_{c}\right)$ with $M_{c}$ given by (4.6), a is a connection on $P_{\text {det }}$ such that $F_{a}^{(0,2)}=0$ and $\hat{a}$ is generated by a and the Levi-Civita connection.

We would like to stress that the operator $\bar{\partial}_{\hat{a}}$ is determined by both the Levi-Civita connection and the connection $a$.

\subsection{Twisted holomorphic curves and solutions to Seiberg-Witten equations}

Below we would like to describe a system of equations introduced by Cieliebak, Gaio and Salamon in [18] for Riemannian surfaces and independently by Mundet i Riera in [40] for arbitrary Kähler manifolds. To define the above mentioned equations we need two Kähler manifolds, which will play a different role: a source manifold $K$ and target one $F$. Pick an $S^{1}$-principal bundle $P_{0}$ over the source $K$ with a holomorphic structure $a$ meaning a connection with vanishing $(0,2)$-part of the curvature. Suppose also that the target $F$ admits an $S^{1}$-symmetry with momentum map $\mu_{F}: F \rightarrow \mathbb{R}$. Consider the system of PDE for the pair $(a, \Phi)$ :

$$
\left\{\begin{array}{l}
\bar{\partial}_{a} \Phi=0, \\
\Lambda F_{a}+\mu_{F} \circ \Phi=0 .
\end{array}\right.
$$

We will refer to equations 4.8 as a THC-system for brevity. Note that the system (4.8) is called symplectic vortex equations in [18] and we will reserve that name for the case when the source manifold is a Riemann surface, as it is in [18].

We will also consider moduli spaces associated to the THC-system. To introduce them remark that the gauge group $\mathcal{G}=\operatorname{Map}\left(X, S^{1}\right)$ acts on the space of solutions to 4.8 . Denote $\mathcal{F}=P_{0} \times_{S^{1}} F$ and set

$\mathcal{M}_{\mathrm{THC}}=\left\{(a, \Phi) \in \mathcal{A}_{h}\left(P_{0}\right) \times \Gamma(\mathcal{F}) \mid(a, \Phi)\right.$ is a solution of $\left.4.8,[\Phi]=\mathrm{c}\right\} / \mathcal{G}$,

where $\mathcal{A}_{h}\left(P_{0}\right)$ denote the space of holomorphic structures on a principle bundle $P_{0}$ and $\mathrm{c}$ is an equivariant homotopy class of maps. The space defined above depends on the choice of a principal bundle $P_{0}$, a fiber $F$ and a homotopy class c, i.e. $\mathcal{M}_{T H C}=\mathcal{M}_{T H C}\left(P_{0}, F, c\right)$. However, we will write simply $\mathcal{M}_{T H C}$ for the sake of brevity if it does not make a confusion.

In general the space $\mathcal{M}_{T H C}$ will have singularities. However, there are two common approaches to remain in the smooth category. One may consider

\footnotetext{
$\mathrm{b}$ abbreviation of twisted holomorphic curve
} 
perturbed equations and prove that for a generic choice of perturbations the moduli space will be smooth (see [40] for details) or consider only irreducible solutions, i.e. those pairs whose stabilizer is trivial. In the latter case we denote the moduli space by $\mathcal{M}_{T H C}^{*}$.

Remark 4.3.1. In order to guarantee that the moduli space $\mathcal{M}_{T H C}$ is a manifold one has to pass from spaces of smooth sections to appropriate Sobolev completions (see [40] for details). It follows from the ellipticity of the system in question that it not essential what particular Sobolev completion is chosen. Since this technique is well-known and documented we will not mention that an appropriate Sobolev completion has to be chosen but rather describe our results and constructions in terms of smooth data.

Although systems (4.7) and (4.8) look very similar there is a significant difference: while the map $u$ is defined on the $H=\left(S^{1} \times S^{1}\right) / \pm 1$ principal bundle $P_{\text {red }}$ and the operator $\bar{\partial}_{\hat{a}}$ makes use of the Levi-Civita connection, the domain of $\Phi$ is only an $S^{1}$-principal bundle and the operator $\bar{\partial}_{a}$ requires just a choice of connection on $P_{0}$. However it seems natural to expect that these two systems are closely related and we will work out details in the sequel.

Remark 4.3.2 (Seiberg-Witten equations on Kähler manifolds with trivial canonical bundle). Probably the easiest way to see a direct connection between systems (4.7) and (4.8) is the following. Assume for a moment that the canonical bundle of the source Kähler surface $X$ is trivial as well as that its connection, induced by the Levi-Civita connection, is also trivial. For example, $X$ can be a K3 surface or flat torus $\mathbb{T}^{4}$. Under such circumstances, the bundle $\left(P_{\text {red }}, \hat{a}\right)$ further reduces to $\left(P_{\text {det }}, a\right)$ so that equations (4.7) reduce to the THC-system with $K=X, P_{0}=P_{\text {det }}, \Phi=u$.

Another connection between systems (4.7) and (4.8) is based on the following observation. Let $Z_{c}$ be the Kähler reduction of $M_{c}$ (see (4.6)) with respect to $S_{r}^{1}: Z_{c}=M_{c} / / S_{r}^{1}=M_{c} / \mathbb{C}_{r}^{*}$. It is known [11] that if a hyperKähler manifold $M$ admits a permuting $\mathbb{H}^{*}$-action and $\mathbb{C}_{r}^{*} \subset \mathbb{H}^{*}$ is a standard embedding, then $Z(N)=M / \mathbb{C}_{r}^{*}$ is a twistor space of quaternionic Kähler manifold $N=M / \mathbb{H}^{*}$. The map $\mu_{c}$ satisfies $\mu_{c}(z \cdot m)=z^{2} \mu_{c}(m)$ and therefore does not descend to $Z(N)$. However it gives rise to a section $\mu_{c}^{\prime}$ of a line bundle $\mathcal{L}^{2}$, where $\mathcal{L} \rightarrow Z(N)$ is defined as $\mathcal{L}=(M \times \mathbb{C}) / \mathbb{C}_{r}$ with respect to the following action of $\mathbb{C}_{r}^{*}: z \cdot(m, w)=(z m, z w)$. It follows that the zero-locus of $\mu_{c}^{\prime}$ is well defined and it is easy to see that it coincides with $Z_{c} \subset Z(N)$.

Let $\Phi$ be the projection of $u$ onto $Z_{c}$. It follows that $\Phi \in \operatorname{Map}^{S_{0}^{1}}\left(P_{\text {det }}, Z_{c}\right)$ and one can think about $\Phi$ as a section of the "determinant bundle" $\mathcal{Z}_{c}=$ $P_{\text {det }} \times{ }_{S_{0}^{1}} Z_{c}$. Observe that Theorem 4.2.3 implies that the connection $a$ induces 
a holomorphic structure on $\mathcal{Z}_{c}$ so it makes sense to talk about holomorphic sections of $\mathcal{Z}_{c}$. Further, one may consider $M_{c}$ as the total space of a Hermitian line bundle $\mathcal{L} \rightarrow Z_{c}$ so that the map $u$ decomposes as a pair $(\Phi, s)$, where

$$
s \in \Gamma\left(K_{X}^{-1} \otimes \pi_{!}^{\operatorname{det}}\left(\Phi^{*} \mathcal{L}\right)\right) .
$$

The manifold $Z_{c}$ inherits a Kähler structure from $M_{c}$. Then the holomorphic action of $S_{0}^{1}$ gives rise to a moment map $\tau: Z_{c} \rightarrow \mathbb{R}$. It is easy to see that $\mu_{1} \circ u=|s|^{2} \tau \circ \Phi$. Further, the holomorphicity of $u$ implies that both $\Phi$ and $s$ are holomorphic and the second equation of (4.7) takes the form

$$
\Lambda F_{a}+|s|^{2} \tau \circ \Phi=0 .
$$

It may happen that for some choice of an equivariant homotopy class c of maps $\Phi$ the bundle $K_{X}^{-1} \otimes \pi_{!}^{\operatorname{det}}\left(\Phi^{*} \mathcal{L}\right)$ becomes trivial. It follows that in this case $s$ is necessarily a constant so that the pair $(a, \Phi)$ is a solution of (4.8) for a source manifold $X, P_{0}=P_{\text {det }}$ and $F=Z_{c}$.

\subsection{Seiberg-Witten equations as THC- system with additional twisting}

Since the celebrated papers of Gromov lots of efforts were made to study the space of holomorphic maps from an almost complex manifold (mainly Riemann surface) into another one (most often symplectic). Good understanding of such spaces provides a lot of information about the chosen manifolds.

A natural question arises: what can be taken as a "twisted version" of holomorphic curves? There seem to be two natural approaches. The first is to fix an $S^{1}$-principal bundle $P_{0}$ (we consider here only the case of $S^{1}$-symmetry, although for the definition one may equally well take an arbitrary compact Lie group) and also fix a connection with vanishing $(0,2)$-component of the curvature. Then, by the analogy with holomorphic curves, one can study the space of equivariant holomorphic maps ${ }^{\mathrm{C}}$ into a target manifold $F$, which must admit a holomorphic $S^{1}$-action, i.e. the space of solutions to

$$
\bar{\partial} u=0, \quad u \in \operatorname{Map}^{S^{1}}\left(P_{0}, F\right) .
$$

The other approach is to consider equations where the unknowns are both the connection and the holomorphic map, for example THC-system 4.8).

With this understood we may combine both approaches in the following sense. Pick two $S^{1}$-principal bundles $P_{0}$ and $P_{1}$ over a source Kähler manifold

${ }^{\mathrm{c}}$ see Convention 3.2 .19 and the preceding discussion 
$X$ and fix a connection with vanishing $(0,2)$-part of the curvature on one of them, say $P_{1}$. Put $P=P_{0} \times P_{1}$. Now a target manifold $F$ must admit $\mathbb{T}^{2}=S^{1} \times S^{1}$-symmetry and let $\mu_{F}$ denote momentum map of the first component of $\mathbb{T}^{2}$. Then we may consider the following problem

$$
\left\{\begin{array}{l}
\bar{\partial}_{\hat{a}} u=0, \quad u \in \operatorname{Map}^{\mathbb{T}^{2}}(P, F), \\
\Lambda F_{a}+\mu_{F} \circ u=0,
\end{array}\right.
$$

where $a$ is a connection on $P_{0}$, which (together with the fixed connection on $P_{1}$ ) equips $P$ with a holomorphic structure $\hat{a}$. Equations of such form (for general compact Lie groups) were introduced by Okonek and Telemann [58, where they are called vortex type equations. Thus we see that SeibergWitten equations on Kähler surfaces reduce to vortex type equation for $P_{0}=$ $P_{\text {det }}, P_{1}=P_{r}$. Although in general one is free to choose any bundle $P_{1}$ as well as any "parameter" connection on $P_{1}$, however the setting of Seiberg-Witten theory prescribes that choice: $P_{1}$ has to be principal bundle of anticanonical line bundle and connection has to be induced by the Levi-Civita one.

\subsection{THC equations over products of holomor- phic curves}

In this section we will consider the THC equations $(4.8)$ in the case when the source manifold $X$ is a product of two holomorphic curves: $X=\Sigma \times \mathrm{S}$. The author was inspired by ideas of Cieliebak, Gaio, Mundet i Riera and Salamon [17], where the classical Seiberg-Witten equations are considered.

Although the approach described below is interesting on its own, we mainly regard the THC-system as a prototype of the Seiberg-Witten equations (4.7) over Kähler surfaces. Since the exposition slightly simplifies for the THC-system, we start with the latter. However, before we start the discussion of properties of the system (4.8) we would like to make a few observations.

Gauge group. Let $Y$ be a connected manifold. Consider a group $\mathcal{G}_{Y}=$ $\operatorname{Map}\left(Y, S^{1}\right)$. For any $g \in \mathcal{G}_{Y}$ we have an induced homomorphism $g_{*}: \pi_{1}(Y) \rightarrow$ $\pi_{1}\left(S^{1}\right) \cong \mathbb{Z}$, which descends to $\pi_{1}(Y) /\left[\pi_{1}(Y), \pi_{1}(Y)\right] \cong H_{1}(Y ; \mathbb{Z}) \rightarrow \mathbb{Z}$. In other words, $g_{*}$ gives rise to an element of $H^{1}(Y ; \mathbb{Z})$, i.e. we have a homomorphism $\mathcal{G}_{Y} \rightarrow H^{1}(Y ; \mathbb{Z})$. The above homomorphism parameterizes connected components of $\mathcal{G}_{Y}$, i.e. $\mathcal{G}_{Y}=\mathcal{G}_{Y}^{0} \times H^{1}(Y ; \mathbb{Z})$, where $\mathcal{G}_{Y}^{0}$ denotes the identity component. The equivalent definition of the subgroup $\mathcal{G}_{Y}^{0}$ is as follows: $\mathcal{G}_{Y}^{0}$ consists of those elements $g$, whose associated group-homomorphisms $g_{*}: \pi_{1}(Y) \rightarrow \pi_{1}\left(S^{1}\right)$ are trivial. 
Pick a point $y \in Y$ and denote $\mathcal{G}_{Y, y}^{0}=\left\{g \in \mathcal{G}_{Y}^{0} \mid g(y)=1\right\}$. It follows from the above observation that any $g \in \mathcal{G}_{Y, y}^{0}$ has a unique lift $\xi$ to the universal cover $\mathbb{R}$ of the circle such that $\xi(y)=0$. Since the space $\mathbb{R}$ is contractible, $\mathcal{G}_{Y, y}^{0}$ has the same property. We have $\mathcal{G}_{Y}^{0}=\mathcal{G}_{Y, y}^{0} \times S^{1}$ and therefore

$$
\mathcal{G}_{Y}=\mathcal{G}_{Y, y}^{0} \times S^{1} \times H^{1}(Y ; \mathbb{Z}) .
$$

If the Riemannian metric is fixed on $Y$, then one can identify the group $\mathcal{G}_{Y, y}^{0}$ with

$$
\tilde{\mathcal{G}}_{Y}=\left\{g \in \mathcal{G}_{Y}^{0} \mid g=e^{i \xi}, \int_{Y} \xi d v o_{Y}=0\right\} .
$$

In particular, $\tilde{\mathcal{G}}_{Y}$ is also contractible and we have a similar decomposition

$$
\mathcal{G}_{Y}=\tilde{\mathcal{G}}_{Y} \times S^{1} \times H^{1}(Y ; \mathbb{Z})
$$

Symplectic vortex equations and their modification. Let $P_{0} \rightarrow \Sigma$ be a principal $S^{1}$-bundle. Consider symplectic vortex equations over $\Sigma$ :

$$
\left\{\begin{array}{l}
\bar{\partial}_{b} \Psi=0, \\
*_{\Sigma} F_{b}+\mu_{F} \circ \Psi=0,
\end{array}\right.
$$

where $b$ is a connection on $P_{0}$ and $\Psi: P_{0} \rightarrow F$ is an $S^{1}$-equivariant map. Denote by $\mathcal{M}_{S V}^{0}$ the moduli space corresponding to the problem 4.11), i.e.

$$
\mathcal{M}_{S V}^{0}=\left\{(b, \Psi) \in \mathcal{A}\left(P_{0}\right) \times \operatorname{Map}^{S^{1}}\left(P_{0}, F\right) \mid(b, \Psi) \text { solves 4.11) }\right\} / \mathcal{G}_{\Sigma}^{0} .
$$

Remark 4.5.1. Usually one defines the moduli space as the space of solutions modulo the whole gauge group (in our case $\mathcal{G}_{\Sigma}$ ). However it is more convenient for us to divide only by the identity component $\mathcal{G}_{\Sigma}^{0}$ of the gauge group; the superscript " 0 " is used to keep in mind that we have chosen the subgroup $\mathcal{G}_{\Sigma}^{0}$ rather then the whole group. Notice also that the group $\mathcal{G}_{\Sigma} / \mathcal{G}_{\Sigma}^{0}$ acts on $\mathcal{M}_{S V}^{0}$ and its factor is $\mathcal{M}_{S V}$.

Remark 4.5.2. It is well-known (see [18] for example) that the space $\mathcal{M}_{S V}^{0}$ can be obtained as an (infinite-dimensional) Kähler reduction provided the target manifold $F$ is not only symplectic, but also Kähler, and, consequently, $\mathcal{M}_{S V}^{0}$ is also a Kähler manifold.

Indeed, first observe that the space $\mathcal{A}\left(P_{0}\right)$ carries a natural infinite-dimensional Kähler structure. This easily follows from the fact that the tangent space to $\mathcal{A}\left(P_{0}\right)$ at any point may be identified with $\Omega^{1}(\Sigma)$ and we may define 
a complex structure by the Hodge star operator $*_{\Sigma}: \Omega^{1}(\Sigma) \rightarrow \Omega^{1}(\Sigma)$. Under the above conventions the symplectic form is

$$
\omega_{\mathcal{A}(P)}\left(\varphi_{1}, \varphi_{2}\right)=\int_{\Sigma} \varphi_{1} \wedge \varphi_{2}, \quad \varphi_{1}, \varphi_{2} \in \Omega^{1}(\Sigma)
$$

On the other hand, we have the following general phenomenon: the space of sections $\Gamma\left(P_{G} \times_{G} Z\right) \cong \operatorname{Map}^{G}\left(P_{G}, F\right)$ has a natural complex structure, where $\pi: P_{G} \rightarrow X$ is a $G$-principal bundle and $F$ is a complex manifold, provided $G$ acts holomorphically on $F$. Indeed, fix a map $\Psi$. Then the tangent space to $\operatorname{Map}^{G}\left(P_{G}, F\right)$ at the point $\Psi$ is the complex vector space $\Gamma\left(\pi_{!}\left(\Psi^{*} T F\right)\right)$. Further, when $F$ is Kähler, then $\Gamma\left(\pi_{!}\left(\Phi^{*} T F\right)\right)$ inherits the $L^{2}$-scalar product, provided $G$ also acts isometrically. Hence, in this case $\operatorname{Map}^{G}\left(P_{G}, F\right)$ is also endowed with the Kähler structure.

Thus the space $\operatorname{Map}^{S^{1}}\left(P_{0}, F\right)$ carries a natural Kähler structure and therefore so does $\mathcal{A}\left(P_{0}\right) \times \operatorname{Map}^{S^{1}}\left(P_{0}, F\right)$. Now the space of solutions to the first equation of 4.11 defines a complex submanifold $Z=\left\{\bar{\partial}_{b} \Psi=0\right\} \subset \mathcal{A}\left(P_{0}\right) \times$ $\operatorname{Map}^{S^{1}}\left(P_{0}, F\right)$, which is invariant under the $\mathcal{G}_{\Sigma}$-action. Further, it is easy to check that the momentum map $\mathrm{m}: \mathcal{A}\left(P_{0}\right) \times \operatorname{Map}^{S^{1}}\left(P_{0}, F\right) \rightarrow \operatorname{Lie}\left(\mathcal{G}_{\Sigma}\right) \cong$ $\operatorname{Map}(\Sigma, \mathbb{R})$ of the gauge group $\mathcal{G}_{\Sigma}$ is given by the right-hand side of the second equation of (4.11). Therefore, the Kähler reduction of $Z$ coincides with the moduli space $\mathcal{M}_{S V}$.

Below we will also use another modification of the moduli space, namely a Kähler reduction of $Z \subset \mathcal{A}\left(P_{0}\right) \times \operatorname{Map}^{S^{1}}\left(P_{0}, F\right)$ with respect to the subgroup $\tilde{\mathcal{G}}_{\Sigma} \subset \mathcal{G}_{\Sigma}^{0}$. In this case, the momentum map $\tilde{\mu}$ is given by

$$
\tilde{\mu}(b, \Psi)=*_{\Sigma} F_{b}+\mu_{F} \circ \Psi-\frac{1}{\operatorname{Vol}(\Sigma)} \int_{\Sigma} \mu_{F} \circ \Psi d v o_{\Sigma}
$$

and the role of equations 4.11 now plays the following system

$$
\left\{\begin{array}{l}
\bar{\partial}_{b} \Psi=0, \\
*_{\Sigma} F_{b}+\mu_{F} \circ \Psi-\frac{1}{\operatorname{Vol}(\Sigma)} \int_{\Sigma} \mu_{F} \circ \Psi d v \circ l_{\Sigma}=\frac{d}{\operatorname{Vol}(\Sigma)}+\tau,
\end{array}\right.
$$

where $d=\operatorname{deg} P_{0}$ and $\tau \in \mathbb{R}$ is a parameter. Therefore we define $\biguplus^{\mathrm{d}}$

$$
\tilde{\mathcal{M}}_{S V}=\left\{(b, \Psi) \in \mathcal{A}\left(P_{0}\right) \times \operatorname{Map}^{S^{1}}\left(P_{0}, F\right) \mid(b, \Psi) \text { solves 4.14) }\right\} / \tilde{\mathcal{G}}_{\Sigma} .
$$

Observe that the group $\mathcal{G}_{\Sigma}^{0} / \tilde{\mathcal{G}}_{\Sigma} \cong S^{1}$ acts on $\tilde{\mathcal{M}}_{S V}$ and the corresponding symplectic reduction is $\mathcal{M}_{S V}^{0}$. In the context of Donalson's theory the analogue of $\tilde{\mathcal{M}}_{S V}$ is known as the moduli space of framed instantons [20].

\footnotetext{
${ }^{\mathrm{d}}$ we suppress the dependence of $\tilde{\mathcal{M}}_{S V}$ on $\tau$ in notations
} 
Let $P_{\Sigma}$ and $P_{\mathrm{S}}$ be $S^{1}$-principal bundles over $\Sigma$ and $\mathrm{S}$ correspondingly. Denote by $L_{\Sigma}$ and $L_{\mathrm{S}}$ the corresponding complex line bundles. Recall that $X$ is a product of $\Sigma$ and $\mathrm{S}$ considered with its product metric and complex structure. Thus we have two natural projections: $\pi_{\Sigma}: X \rightarrow \Sigma$ and $\pi_{\mathrm{S}}: X \rightarrow$ S. Denote $L_{\text {det }}=\pi_{\Sigma}^{*} L_{\Sigma} \otimes \pi_{\mathrm{S}}^{*} L_{\mathrm{S}}$ and let $P_{\text {det }}$ be its principal bundle. One can also describe the principal bundle $P_{\text {det }}$ directly as follows

$$
P_{\text {det }}=\left(\pi_{\Sigma}^{*} P_{\Sigma} \times \pi_{\mathrm{S}}^{*} P_{\mathrm{S}}\right) / S^{1},
$$

where the action of $S^{1}$ is given by $\left(z, p_{\Sigma}, p_{\mathrm{s}}\right) \mapsto\left(p_{\Sigma} z, p_{\mathrm{s}} \bar{z}\right)$.

Pick reference connections $b_{0}$ and $c_{0}$ on $P_{\Sigma}$ and $P_{\mathrm{S}}$ correspondingly. They define a connection $a_{0}$ on $P_{\text {det }}$. Then, for an arbitrary connection $a$ on $P_{\text {det }}$, we have $a-a_{0}=\alpha \in \Omega^{1}(\Sigma \times \mathrm{S})$. Since

$$
\Omega^{1}(\Sigma \times \mathrm{S}) \cong \Omega^{1}(\Sigma) \otimes \Omega^{0}(\mathrm{~S}) \oplus \Omega^{0}(\Sigma) \otimes \Omega^{1}(\mathrm{~S}),
$$

we can decompose $\alpha$ into two components: $\alpha=\beta+\gamma$. Further, denote $b=b_{0}+\beta$ and $c=c_{0}+\gamma$. One can think about $b$ as a family of connections on $P_{\Sigma}$ parameterized by the curve S; symmetrically, $c$ can be thought of as a family of connections on $P_{\mathrm{S}}$ parameterized by the curve $\Sigma$. We say that $b$ and $c$ are components of the connection $a$.

The curvature $F_{a}$ of the connection $a$ can be written as $F_{a_{0}}+d_{X} \alpha \in$ $\Omega^{2}(\Sigma \times \mathrm{S})$. Further, $d_{X} \alpha=d_{\Sigma} \beta+d_{\Sigma} \gamma+d_{\mathrm{S}} \beta+d_{\mathrm{S}} \gamma$. The Künneth formula provides a decomposition of $\Omega^{2}(X)$ similar to 4.16), namely

$$
\Omega^{2}(\Sigma \times \mathrm{S}) \cong \Omega^{2}(\Sigma) \otimes \Omega^{0}(\mathrm{~S}) \oplus \Omega^{1}(\Sigma) \otimes \Omega^{1}(\mathrm{~S}) \oplus \Omega^{0}(\Sigma) \otimes \Omega^{2}(\mathrm{~S}) .
$$

Therefore $\left(d_{X} \alpha\right)_{(2,0)}=d_{\Sigma} \beta,\left(d_{X} \alpha\right)_{(1,1)}=d_{\Sigma} \gamma+d_{\mathrm{S}} \beta$ and $\left(d_{X} \alpha\right)_{(0,2)}=d_{\mathrm{S}} c$. Since $F_{a_{0}}=F_{b_{0}}+F_{c_{0}}$, we obtain $\left(F_{a}\right)_{(2,0)}=F_{b},\left(F_{a}\right)_{(0,2)}=F_{c}$.

Observe that $\Lambda\left(F_{a}\right)_{(1,1)}=0$. Indeed this follows from the decomposition (4.17) and the fact that

$$
\omega_{X}=\omega_{\Sigma}+\omega_{\mathrm{S}}
$$

belongs to $\Omega^{2}(\Sigma) \otimes \Omega^{0}(\mathrm{~S}) \oplus \Omega^{0}(\Sigma) \otimes \Omega^{2}(\mathrm{~S})$. Moreover, it follows that

$$
\Lambda F_{a}=*_{\Sigma} F_{b}+*_{S} F_{c} .
$$

Let $s+i t: U \rightarrow \mathbb{C}$ be a local coordinate on $U \subset \mathrm{S}$ and assume that a trivialization of $P_{\mathrm{S}}$ over $U$ is chosen. Then the connection $c$ can be written as a local $1-$ form $c_{1} d s+c_{2} d t$.

Remark also that any connection over a holomorphic curve defines a holomorphic structure since the $(0,2)$ part (relative to the Hodge decomposition) of the curvature automatically vanishes. However this is no longer the case 
when the base manifold $X$ is a Kähler surface, but it is well known (see [29] for example) that the Hodge $(0,2)$ part of $F_{a}$ vanishes if and only if

$$
\partial_{s} b-d_{\Sigma} c_{1}+*_{\Sigma}\left(\partial_{t} b-d_{\Sigma} c_{2}\right)=0,
$$

where $\partial_{s}$ and $\partial_{t}$ denote partial derivatives with respect to variables $s$ and $t$ respectively.

It follows from 4.15) that we have a natural isomorphism

$$
\operatorname{Map}^{S^{1}}\left(P_{\text {det }}, F\right) \cong \operatorname{Map}^{\mathbb{T}^{2}}\left(\pi_{\Sigma}^{*} P_{\Sigma} \times \pi_{\mathrm{S}}^{*} P_{\mathrm{S}}, F\right),
$$

where the torus $\mathbb{T}^{2}$ acts on $F$ via the homomorphism $\mathbb{T}^{2} \rightarrow S^{1},\left(z_{1}, z_{2}\right) \mapsto$ $z_{1} z_{2}$. Further,

$$
\operatorname{Map}^{\mathbb{T}^{2}}\left(\pi_{\Sigma}^{*} P_{\Sigma} \times \pi_{\mathrm{S}}^{*} P_{\mathrm{S}}, F\right) \cong \operatorname{Map}^{S^{1}}\left(P_{\mathrm{S}}, \operatorname{Map}^{S^{1}}\left(P_{\Sigma}, F\right)\right) .
$$

Indeed, if $\Phi \in \operatorname{Map}^{\mathbb{T}^{2}}\left(\pi_{\Sigma}^{*} P_{\Sigma} \times \pi_{\mathrm{S}}^{*} P_{\mathrm{S}}, F\right)$ then the above isomorphism is the following map

$$
\Phi \mapsto\left(p \mapsto \Phi_{p}=\left.\Phi\right|_{P_{\Sigma} \times\{p\}}\right), \quad p \in P_{\mathrm{S}}
$$

One can regard $\Phi_{p}$ as a family of equivariant maps $P_{\Sigma} \rightarrow F$ parameterized by $P_{\mathrm{S}}$.

Summing up we get

$$
\operatorname{Map}^{S^{1}}\left(P_{\text {det }}, F\right) \cong \operatorname{Map}^{S^{1}}\left(P_{\mathrm{S}}, \operatorname{Map}^{S^{1}}\left(P_{\Sigma}, F\right)\right) .
$$

Next, recall that the operator $\bar{\partial}_{a}$ is defined by the following sequence

$$
\Gamma\left(P_{\text {det }} \times_{S^{1}} F\right) \stackrel{\nabla^{a}}{\longrightarrow} \Omega^{1}(X) \otimes \Gamma\left(\pi_{!}^{\text {det }}\left(\Phi^{*} T F\right)\right) \rightarrow \Omega^{0,1}(X) \otimes \Gamma\left(\pi_{!}^{\text {det }}\left(\Phi^{*} T F\right)\right) .
$$

Now it follows from 4.16) that we have a decomposition $\Omega^{0,1}(X)=\Omega^{0,1}(\Sigma) \oplus$ $\Omega^{0,1}(S)$. But this implies that both maps $\Phi_{p}$ (for a fixed $p \in P_{\mathrm{S}}$ ) and $p \mapsto$ $\Phi_{p}$ are holomorphic in the following sense. The map $\Phi_{p} \in \operatorname{Map}^{S^{1}}\left(P_{\Sigma}, F\right)$ is a holomorphic section of the corresponding bundle with respect to the connection $b$ (for a fixed $p \in P_{\mathrm{S}}$ ) while the map $P_{\mathrm{S}} \rightarrow \operatorname{Map}^{S^{1}}\left(P_{\Sigma}, F\right), \quad p \mapsto \Phi_{p}$ is holomorphic with respect to the holomorphic structure on $\operatorname{Map}^{S^{1}}\left(P_{0}, F\right)$ described in Remark 4.5.2 and the connection $c$ on $P_{\mathrm{S}}$. More precisely, the following equations are satisfied

$$
\begin{aligned}
& \bar{\partial}_{b} \Phi_{p}=0, \\
& \partial_{s} \Phi+c_{1} K(\Phi)+I_{F}\left(\partial_{t} \Phi+c_{2} K(\Phi)\right)=0,
\end{aligned}
$$

where $K(\mathrm{f})$ denotes the Killing vector field of the $S^{1}$-action on $F$ at the point $f$. 
Proposition 4.5.3. Let $s+i t$ be a local coordinate on $\mathrm{S}$. The pair $(a, \Phi) \in$ $\mathcal{A}\left(P_{\text {det }}\right) \times \Gamma(\mathcal{F})$ is a solution of the THC-system (4.8) with $\left(F_{a}\right)^{0,2}=0$ over $X=\Sigma \times \mathrm{S}$ if and only if the following system holds

$$
\left\{\begin{array}{l}
\bar{\partial}_{b} \Phi=0 \\
\partial_{s} \Phi+c_{1} K(\Phi)+I_{F}\left(\partial_{t} \Phi+c_{2} K(\Phi)\right)=0 \\
\partial_{s} b-d_{\Sigma} c_{1}+*_{\Sigma}\left(\partial_{t} b-d_{\Sigma} c_{2}\right)=0 \\
*_{\Sigma} F_{b}+*_{s} F_{c}+\mu_{F} \circ \Phi=0
\end{array}\right.
$$

where $b$ and $c$ are components of a (see discussion after (4.16)) and $c_{1} d s+c_{2} d t$ is a local representation of the connection $c$.

We would like to stress that the system 4.20 was obtained under the assumption that the metric on $\Sigma \times \mathrm{S}$ is the product metric: $g_{X}=g_{\Sigma} \oplus g_{\mathrm{S}}$. However one may equally well work with different metrics, for example

$$
g_{X}^{\varepsilon}=g_{\Sigma} \oplus \varepsilon^{-1} g_{\mathrm{S}},
$$

where $\varepsilon \in(0,1]$ is a parameter. We also assume that the complex structure is $I_{\Sigma} \oplus I_{\mathrm{S}}$ and consequently the symplectic form is given by $\omega_{X}^{\varepsilon}=\omega_{\Sigma} \oplus \varepsilon^{-1} \omega_{\mathrm{S}}$. Then

$$
\Lambda^{\varepsilon} F_{a}=*_{\Sigma} F_{b}+\varepsilon *_{S} F_{c}
$$

and therefore the system analogous to 4.20 for the metric (4.21) takes the following form

$$
\left\{\begin{array}{l}
\bar{\partial}_{b} \Phi=0 \\
\partial_{s} \Phi+c_{1} K(\Phi)+I_{F}\left(\partial_{t} \Phi+c_{2} K(\Phi)\right)=0 \\
\partial_{s} b-d_{\Sigma} c_{1}+*_{\Sigma}\left(\partial_{t} b-d_{\Sigma} c_{2}\right)=0 \\
*_{\Sigma} F_{b}+\varepsilon *_{S} F_{c}+\mu_{F} \circ \Phi=0 .
\end{array}\right.
$$

Hypothesis 4.5 .4 ("Adiabatic limit conjecture"). There is a one-toone correspondence between solutions of the system (4.20) and solutions of the following equations

$$
\left\{\begin{array}{l}
\bar{\partial}_{b} \Phi=0 \\
\partial_{s} \Phi+c_{1} K(\Phi)+I_{F}\left(\partial_{t} \Phi+c_{2} K(\Phi)\right)=0 \\
\partial_{s} b-d_{\Sigma} c_{1}+*_{\Sigma}\left(\partial_{t} b-d_{\Sigma} c_{2}\right)=0 \\
*_{\Sigma} F_{b}+\mu_{F} \circ \Phi=0
\end{array}\right.
$$


Remark 4.5.5. The system 4.20 is obtained from 4.22 for $\varepsilon=1$; on the other hand, one obtains 4.23 by formally setting $\varepsilon=0$. The above hypothesis then asserts that the "limiting problem" (4.23) is equivalent to the perturbed one (4.22).

The idea of adiabatic limit first appeared in Atiyah's work [4]. Its modification, which is very similar to the one stated above, was carried out by Dostoglou and Salamon in [22] and led to a connection between self-dual instantons and pseudo-holomorphic curves. Further developments and modifications of the adiabatic limit technique may be found in [17, 30, 14, 57].

Let us return for a while to the symplectic vortex equations (4.11). As we have already remarked, one can obtain $\mathcal{M}_{S V}^{0}$ as a symplectic reduction of $\tilde{\mathcal{M}}_{S V}$ with respect to the action of $S^{1} \cong \mathcal{G}_{\Sigma}^{0} / \tilde{\mathcal{G}}_{\Sigma}$. In this case the momentum map is

$$
\nu([a, \Psi])=\frac{1}{\operatorname{Vol}(\Sigma)} \int_{\Sigma} \mu_{F} \circ \Psi{d v o l_{\Sigma}}
$$

We assume that $\tau$ is chosen such that the action of $S^{1}$ on

$$
P_{S V}=\nu^{-1}\left(-\frac{d}{\operatorname{Vol}(\Sigma)}-\tau\right)
$$

is free so that $P_{S V} \rightarrow \mathcal{M}_{S V}^{0}$ is an $S^{1}$-principal bundle. Moreover, it comes equipped with a connection. Indeed, since the moduli space $\tilde{\mathcal{M}}_{S V}$ is a Kähler reduction, it inherits in particular an $S^{1}$-invariant metric. Then one obtains the horizontal distribution on $P_{S V} \subset \tilde{\mathcal{M}}_{S V}$ as the orthogonal complement to the distribution of Killing vector fields (for more details see Section 2.1.1).

Theorem 4.5.6. Let $X$ be a product of two holomorphic curves $\Sigma \times \mathrm{S}$ and $P_{d e t} \rightarrow X$ be given by (4.15). Suppose that Hypothesis 4.5.4 holds. Then there is one-to-one correspondence between holomorphic curves $f: \mathrm{S} \rightarrow \mathcal{M}_{S V}^{0}$ such that $f^{*} P_{S V} \cong P_{\mathrm{S}}$ and the moduli space

$$
\left.\mathcal{M}_{T H C}^{0}=\left\{(a, \Phi) \in \mathcal{A}_{h}\left(P_{0}\right) \times \Gamma(\mathcal{F}) \mid(a, \Phi) \text { is a solution of } 4.8\right)\right\} / \mathcal{G}_{X}^{0},
$$

corresponding to the THC-system (4.8) over $\Sigma \times \mathrm{S}$.

The proof of the above theorem uses two lemmas, which we will prove first.

Let $P_{G} \rightarrow \mathrm{S}$ be a $G$-principal bundle over an (almost) Kähler manifold S (not necessarily a holomorphic curve) with a connection $A$. Let $G$ act on (almost) Kähler manifold $Z$ preserving its Kähler structure. Assume also that an action of another Lie group $\tilde{G}$ on $Z$ is Hamiltonian, preserves Kähler structure of $Z$ and commutes with the action of $G$. Let $\tilde{\mu}: Z \rightarrow \tilde{\mathfrak{g}}$ be its 
momentum map. Suppose a $G$-equivariant map $\psi: P_{G} \rightarrow Z$ satisfies $\tilde{\mu} \circ \psi=$ $\tilde{\tau}$, where $\tilde{\tau}$ is central in $\tilde{\mathfrak{g}}$. Then we have the induced $G$-equivariant map $\varphi: P_{G} \rightarrow \tilde{\mathcal{M}}$, where $\tilde{\mathcal{M}}$ denotes the Kähler reduction of $Z: \tilde{\mathcal{M}}=\tilde{\mu}^{-1}(\tilde{\mathcal{\tau}}) / \tilde{G}$. We assume that $\tilde{\mathcal{M}}$ is a manifold (and in particular a Hausdorff space).

Lemma 4.5.7. Assume that $\tilde{G}$ act freely (or that each point has a finite stabilizer) on $\tilde{\mu}^{-1}(\tilde{\tau})$. The $G$-equivariant map $\varphi$ is holomorphic with respect to the connection $A$ if and only if there exists $\gamma \in \Omega^{1}(\mathrm{~S}) \otimes \tilde{\mathfrak{g}}$ such that the following equation

$$
\left(\psi_{*}-\tilde{K}_{\gamma}(\psi)\right) I_{\mathrm{S}}=I_{Z}\left(\psi_{*}-\tilde{K}_{\gamma}(\psi)\right)
$$

holds on the horizontal distribution $\mathcal{H}$ over $P_{G}$, where $\tilde{K}_{\xi}$ denotes the Killing vector field of the $\tilde{G}$-action corresponding to $\xi \in \tilde{\mathfrak{g}}$.

Proof. Pick a point $p \in P_{G}$ and denote $q=\varphi(p) \in \tilde{\mu}^{-1}(\tilde{\tau}) \subset Z$. Then we have a decomposition

$$
T_{q} Z=\tilde{H}_{q} \oplus \tilde{\mathcal{K}}_{q} \oplus I_{Z} \tilde{\mathcal{K}}_{q}
$$

where $\tilde{\mathcal{K}}$ denotes the distribution spanned by Killing vector fields of the $\tilde{G}^{-}$ action and $\tilde{H}_{q}$ is defined as the orthogonal complement to $\tilde{\mathcal{K}}_{q} \oplus I_{Z} \tilde{\mathcal{K}}_{q}$. It follows in particular that $I_{Z}$ preserves $H_{q}$. Observe also that

$$
T_{q} \tilde{\mu}^{-1}(\tilde{\tau})=H_{q} \oplus \mathcal{K}_{q}
$$

According to the symplectic reduction construction one can identify $\tilde{H}_{q}$ with $T_{[q]} \tilde{\mathcal{M}}$.

Since $\psi$ satisfies the relation $\tilde{\mu} \circ \psi=\tilde{\tau}$, the differential $\psi_{*}$ has components only in $\tilde{H}_{q} \oplus \tilde{\mathcal{K}}_{q}$. Notice that the projection of $\psi_{*}$ to $\tilde{H}$ can be identified with $\varphi_{*}$. Given a horizontal vector $\mathrm{v} \in T_{p} P_{G}$ there exists a unique element $\gamma(\mathrm{v}) \in \tilde{\mathfrak{g}}$ such that $\varphi_{*} \mathrm{v}=\psi_{*} \mathrm{v}-\tilde{K}_{\gamma(\mathrm{v})}(q)$. Therefore, the G-equivariant map $\varphi$ is holomorphic with respect to the connection $A$ iff

$$
\left(\psi_{*}\left(I_{\mathrm{S}} \mathrm{v}\right)-\tilde{K}_{\gamma\left(I_{\mathrm{S}}\right)}(\psi(p))\right)=I_{Z}\left(\psi_{*}(\mathrm{v})-\tilde{K}_{\gamma(\mathrm{v})}(\psi(p))\right) .
$$

Now it follows from the $G$-equivariancy of $\psi$ that $\left(R_{g}\right)^{*} \gamma=\gamma$, where $R_{g}$ : $P_{\mathrm{S}} \rightarrow P_{\mathrm{S}}, p \mapsto p g$. But this means that the 1 -form $\gamma$ descends to $\mathrm{S}$.

Remark 4.5.8. If $\mathrm{S}$ is a holomorphic curve, one can write locally $\psi=$ $\psi(s, t), \quad A=A_{1}(s, t) d s+A_{2}(s, t) d t, \gamma=\gamma_{1}(s, t) d s+\gamma_{2}(s, t) d t$ for a local coordinate $s+i t$ (we also imply that a local trivialization of $P_{G}$ is chosen). Then the equation 4.25 can be rewritten in the following form

$$
\left(\partial_{s} \psi-K_{A_{1}}-\tilde{K}_{\gamma_{1}}(\psi)\right)+I_{Z}\left(\partial_{t} \psi-K_{A_{2}}-\tilde{K}_{\gamma_{2}}(\psi)\right)=0 .
$$


Now assume that the action of a compact Lie group $G$ on $\tilde{\mathcal{M}}$ is Hamiltonian and denote by $\nu: \tilde{\mathcal{M}} \rightarrow \mathfrak{g}$ its momentum map. Suppose also that $G$ acts freely on $\nu^{-1}(\tau)$, where $\tau$ is a central element in $\mathfrak{g}$. Denote by $\mathcal{M}$ the Kähler reduction of $\tilde{\mathcal{M}}: \mathcal{M}=\tilde{\mathcal{M}} / / G=\nu^{-1}(\tau) / G$. Then $Q_{G}=\nu^{-1}(\tau) \rightarrow \mathcal{M}$ is a $G$-principal bundle and it carries the induced connection $B$ as we have already remarked above for the bundle $P_{S V}$. Further, suppose that an equivariant map $\varphi: P_{G} \rightarrow \tilde{\mathcal{M}}$ satisfies $\nu \circ \varphi=\tau$. Finally, let $f: \mathrm{S} \rightarrow \mathcal{M}$ denote the induced map. Obviously, $\varphi$ is an isomorphism between $P_{G}$ and $f^{*} Q_{G}$.

Lemma 4.5.9. If $\varphi$ is a holomorphic equivariant man with respect to the connection $A$, then

- the projection $f: \mathrm{S} \rightarrow \mathcal{M}$ is holomorphic;

- the connection $A$ is the pull-back of $B$.

Proof. Pick a point $p \in P_{G}$ and denote $q=\varphi(p) \in Q_{G} \subset \tilde{\mathcal{M}}$. Let $\mathcal{K}$ denote the distribution of Killing vector fields over $\tilde{\mathcal{M}}$. Similarly as in the proof of the above lemma we have a decomposition

$$
T_{q} \tilde{\mathcal{M}}=H_{q} \oplus \mathcal{K}_{q} \oplus I_{\tilde{\mathcal{M}}} \mathcal{K}_{q}
$$

where $H_{q}$ is the orthogonal complement to $K_{q} \oplus I_{\tilde{\mathcal{M}}} \mathcal{K}_{q}$. In particular subbundle $H$ is $I_{\tilde{\mathcal{M}}}$-invariant. We have also

$$
T_{q} Q_{G}=H_{q} \oplus \mathcal{K}_{q}
$$

One can identify $H_{q}$ with $T_{[q]} \mathcal{M}$.

Let $\mathcal{H}$ be the horizontal distribution over $P_{G}$ corresponding to the connection $A$. Since $\mathcal{H}$ is isomorphic to the pull-back of $T \mathrm{~S}$, the pull-back of the complex structure $I_{\mathrm{S}}$ of $\mathrm{S}$ endows $\mathcal{H}$ with the structure of a complex vector bundle. We do not distinguish between $I_{\mathrm{S}}$ and its pull-back in notation.

Now pick a vector $\mathrm{v} \in \mathcal{H}_{p}$ and denote $\mathrm{w}=\varphi_{*} \mathrm{v} \in T_{q} Q_{G}$. Let $\mathrm{w}^{h}+\mathrm{w}^{v}$ be a decomposition of $\mathrm{w}$ according to (4.27). Since $\varphi$ is holomorphic, we have $\varphi_{*} I_{\mathrm{S}} \mathrm{V}=I_{Z} \varphi_{*} \mathrm{v}=I_{Z} \mathrm{w}^{h}+I_{Z} \mathrm{w}^{v} \in H_{q} \oplus I_{Z} \mathcal{K}_{q}$. On the other hand, the condition $\nu \circ \varphi=\tau$ implies $\varphi_{*} I_{\mathrm{S}} \mathrm{v} \in T_{q} Q_{G}=H_{q} \oplus \mathcal{K}_{q}$. It follows that $I_{Z} \mathrm{w}^{v}=0$, i.e. $\mathrm{w}^{v}=0$. Therefore $\varphi_{*}$ maps horizontal vectors in horizontal ones and this implies that $A$ is the pull-back of $B$. It remains to note that one can identify $f_{*}$ with the projection of $\left.\varphi_{*}\right|_{\mathcal{H}}$ to $H$. Thus the first statement of the lemma also follows.

\footnotetext{
${ }^{\mathrm{e}}$ see Convention 3.2 .19
} 
Remark 4.5.10. It is easy to see, that the inverse statement of the above lemma also holds. Here by the inverse statement we mean the following. Assume that the map $f: \mathrm{S} \rightarrow \mathcal{M}$ is holomorphic. Then we get a tautological equivariant map $\varphi: f^{*} Q_{G} \rightarrow Q_{G}$, which represents a holomorphic section with respect to the connection $f^{*} B$.

Proof of the Theorem 4.5.6. Let $f: S \rightarrow \mathcal{M}_{S V}^{0}$ be a holomorphic curve such that $f^{*} P_{S V} \cong P_{\mathrm{S}}$. Denote by $c^{0}$ the connection on $P_{\mathrm{S}}$ induced from $P_{S V}$ and let $c_{1}^{0} d s+c_{2}^{0} d t$ be its local representation (with respect to a chosen local trivialization). Then the tautological map $\varphi: f^{*} P_{S V} \rightarrow P_{S V} \hookrightarrow \tilde{\mathcal{M}}_{S V}$ is a holomorphic equivariant map $\mathrm{f}^{f}$ with respect to the connection $c^{0}$.

One can consider the space of solutions $\mathcal{S}_{S V}$ to the symplectic vortex equations 4.14 as a $\tilde{\mathcal{G}}_{\Sigma}$-principal bundle over $\tilde{\mathcal{M}}_{S V}$. Since the gauge group $\tilde{\mathcal{G}}_{\Sigma}$ is contractible, the bundle $\mathcal{S}_{S V}$ is trivial, i.e. we have a continuous section $\sigma: \tilde{\mathcal{M}}_{S V} \rightarrow \mathcal{S}_{S V}$. Since any continuous section can be approximated by a smooth one, we can assume that $\sigma$ is smooth. It follows that one can further lift $\varphi$ to a smooth map $\psi: P_{\mathrm{S}} \rightarrow \mathcal{S}_{S V}, \psi(p)=(\Psi(p), b(p)), p \in P_{\mathrm{S}}$. Observe that any two such liftings differ by a gauge transformations.

Applying Lemma 4.5 .7 for $Z=\left\{(b, \Psi) \mid \bar{\partial}_{b} \Psi=0\right\}, \tilde{G}=\tilde{\mathcal{G}}_{\Sigma}, G=S^{1}, \tilde{\tau}=$ $-d / \operatorname{Vol}(\Sigma)-\tau$ we obtain that there exists $\tilde{c} \in \Omega^{1}(\mathrm{~S}) \otimes \operatorname{Lie}\left(\tilde{\mathcal{G}}_{\Sigma}\right)$ such that

$$
\begin{aligned}
& \partial_{s} \Psi+\left(c_{1}^{0}+\tilde{c}_{1}\right) K(\Psi)+I_{F}\left(\partial_{t} \Psi+\left(c_{2}^{0}+\tilde{c}_{2}\right) K(\Psi)\right)=0, \\
& \partial_{s} b-\left(d_{\Sigma} c_{1}^{0}+d_{\Sigma} \tilde{c}_{1}\right)+*_{\Sigma}\left(\partial_{t} b-\left(d_{\Sigma} c_{2}^{0}+d_{\Sigma} \tilde{c}_{2}\right)\right)=0,
\end{aligned}
$$

where $\tilde{c}_{1} d s+\tilde{c}_{2} d t$ is a local representation of $\tilde{c}$. One can interpret $\tilde{c}_{i}$ as functions $U \times \Sigma \rightarrow \mathbb{R}$ such that

$$
\int_{\Sigma} \tilde{c}_{i} d v o l_{\Sigma}=0 \text { for any } s+i t \in U \text {. }
$$

Let us denote $c_{i}=c_{i}^{0}+\tilde{c}_{i}$. Then $c_{1} d s+c_{2} d t \in \Omega^{0}(\Sigma) \otimes \Omega^{1}(\mathrm{~S})$ is a local representation of a family of connections on $P_{\mathrm{S}}$. In these notations, the equations (4.28) have the following form

$$
\begin{aligned}
& \partial_{s} \Psi+c_{1} K(\Psi)+I_{F}\left(\partial_{t} \Psi+c_{2} K(\Psi)\right)=0, \\
& \partial_{s} b-d_{\Sigma} c_{1}+*_{\Sigma}\left(\partial_{t} b-d_{\Sigma} c_{2}\right)=0 .
\end{aligned}
$$

By the construction of $\varphi$ we have $\nu \circ \varphi=d / \operatorname{Vol}(\Sigma)+\tau$, where the map $\nu$ is given by (4.24). Similarly, $\psi=(b, \Psi)$ satisfy $\tilde{\mu} \circ \psi=-d / \operatorname{Vol}(\Sigma)-\tau$ with $\tilde{\mu}$

\footnotetext{
${ }^{\mathrm{f}}$ see Convention 3.2 .19
} 
defined by 4.13). Thus we get

$$
\begin{aligned}
& \bar{\partial}_{b} \Psi=0, \\
& *_{\Sigma} F_{b}+\mu_{F} \circ \Psi-\frac{1}{\operatorname{Vol}(\Sigma)} \int_{\Sigma} \mu_{F} \circ \Psi d \operatorname{vol}_{\Sigma}=\frac{d}{\operatorname{Vol}(\Sigma)}+\tau, \\
& \frac{1}{\operatorname{Vol}(\Sigma)} \int_{\Sigma} \mu_{F} \circ \Psi d \operatorname{vol}_{\Sigma}=-\frac{d}{\operatorname{Vol}(\Sigma)}-\tau,
\end{aligned}
$$

where the first equation just means, that the target of $\psi$ is $Z=\left\{\bar{\partial}_{b} \Psi=0\right\}$.

The space of square-integrable functions $L_{2}(\Sigma)$ can be decomposed into two subspaces: $L_{2}(\Sigma)=\mathbb{R} \oplus \tilde{L}_{2}(\Sigma)$, where the first subspace is generated by the constant function of integral 1 and the other one consists of all functions of zero mean value. Obviously, a function vanishes if and only if both its components vanish. Applying this trick to the function $*_{\Sigma} F_{b}+\mu_{F} \circ \Psi$ we get that the system (4.30) is equivalent to the symplectic vortex equations:

$$
\begin{aligned}
& \bar{\partial}_{b} \Psi=0, \\
& *_{\Sigma} F_{b}+\mu_{F} \circ \Psi=0 .
\end{aligned}
$$

It remains to notice that 4.29) and 4.31 constitute the system 4.23).

Now let us show that a solution of 4.23 defines a holomorphic curve $\mathrm{S} \rightarrow \mathcal{M}_{S V}^{0}$. First, we can decompose functions $c_{i}: U \times \Sigma \rightarrow \mathbb{R}, c_{i}=\tilde{c}_{i}+c_{i}^{0}$, where

$$
c_{i}^{0}=\frac{1}{\operatorname{Vol}(\Sigma)} \int_{\Sigma} c_{i} d v o l_{\Sigma}, \quad \tilde{c}_{i}=c_{i}-\frac{1}{\operatorname{Vol}(\Sigma)} \int_{\Sigma} c_{i} d v o l_{\Sigma} .
$$

Observe that $c_{i}^{0}: U \rightarrow \mathbb{R}, \tilde{c}_{i}: U \times \Sigma \rightarrow \mathbb{R}$ and $\int_{\Sigma} \tilde{c}_{i} d v o l_{\Sigma}=0$. The 1 -form $c_{1}^{0} d s+c_{2}^{0} d t$ is a local representation of a connection $c^{0}$ on $P_{\mathrm{S}}$ while $\tilde{c}_{1} d s+\tilde{c}_{2} d t$ is a local representation of a section $\tilde{c} \in \Omega^{1}(\mathrm{~S}) \otimes \operatorname{Lie}\left(\tilde{\mathcal{G}}_{\Sigma}\right)$. It follows that $\psi(s, t)=(\Phi(s, t), b(s, t))$ represents a map $\psi: P_{\mathrm{S}} \rightarrow \mathcal{S}_{S V}$ in the local coordinate $s+i t$ such that equations (4.28) hold. The other two equations of the system 4.23 can be rewritten in the form 4.30. By Lemma 4.5.7 this implies that we have the induced $S^{1}$-equivariant map $\varphi: P_{\mathrm{S}} \rightarrow \mathcal{\mathcal { M }}_{S V}$ that is holomorphic with respect to the connection $c^{0}$. Moreover, $\varphi$ also satisfies $\nu \circ \varphi=-d / \operatorname{Vol} \Sigma-\tau$. Now Lemma 4.5.9 implies that the induced map $f: \mathrm{S} \rightarrow \mathcal{M}_{S V}^{0}$ is holomorphic and $c^{0}$ is the pull-back of connection on $P_{S V}$. It is clear that gauge-equivalent solutions of 4.23) define the same holomorphic curve $\mathrm{S} \rightarrow \mathcal{M}_{S V}^{0}$. 


\subsection{Seiberg-Witten equations over product of holomorphic curves}

In this section we will consider Seiberg-Witten equations 4.7) in the case when the source manifold $X$ is a product of two holomorphic curves: $X=$ $\Sigma \times \mathrm{S}$. Observe that $P_{\text {red }}$ is a double cover of $P_{r} \times P_{\text {det }}$; if we assume that $[-1,1]=[1,-1] \in H$ acts trivially on $M_{c}$, then

$$
\operatorname{Map}^{H}\left(P_{r e d}, M_{c}\right) \cong \operatorname{Map}^{\mathbb{T}^{2}}\left(P_{r} \times P_{\text {det }}, M_{c}\right)
$$

Let $P_{\text {det }}$ be given by (4.15) as before. Recall that a connection $a$ can be decomposed into the components $b$ and $c$ (see the discussion after (4.16)) and one has $\Lambda F_{a}=*_{\Sigma} F_{b}+*_{S} F_{c}$ for the product metric on $X=\Sigma \times \mathrm{S}$.

From now on assume that $(a, u)$ is a solution of the Seiberg-Witten equations (4.7) and in particular $\left(F_{a}\right)^{(0,2)}=0$. Recall that vanishing of the Hodge $(0,2)$-part of the curvature is equivalent to the equation 4.19). In other words

$$
\left(d_{\mathrm{S}} b-d_{\Sigma} c\right) I_{S}=*_{\Sigma}\left(d_{\mathrm{S}} b-d_{\Sigma} c\right) .
$$

Next, the tangent space of the source manifold $X$ decomposes as $T S \oplus T \Sigma$ and therefore $\Lambda^{2} T X \cong T S \otimes T \Sigma$. It follows that $P_{r}$ is the principal $S^{1}$-bundle of $T S \otimes T \Sigma$. Denote by $P_{T S}$ and $P_{T \Sigma}$ principal $S^{1}$-bundles of $T S$ and $T \Sigma$ correspondingly. Then $P_{r}=\left(P_{T \mathrm{~S}} \times P_{T \Sigma}\right) / S^{1}$, where $S^{1}$ acts by multiplication by $z$ on one factor and by $\bar{z}$ on the other one. Similarly as in the previous section

$$
\operatorname{Map}^{S_{r}^{1} \times S_{0}^{1}}\left(P_{r} \times P_{d e t}, M_{c}\right) \cong \operatorname{Map}^{\mathbb{T}^{4}}\left(P_{T \mathrm{~S}} \times P_{T \Sigma} \times P_{\mathrm{S}} \times P_{\Sigma}, M_{c}\right),
$$

where $\mathbb{T}^{4}$ acts on $M_{c}$ via the homomorphism $\mathbb{T}^{4} \rightarrow S_{r}^{1} \times S_{0}^{1},\left(z_{1}, z_{2}, z_{3}, z_{4}\right) \mapsto$ $\left(z_{1} z_{2}, z_{3} z_{4}\right)$ and pull-backs are suppressed in notations.Further, we have a natural isomorphism

$$
\operatorname{Map}^{\mathbb{T}^{4}}\left(P_{T \mathrm{~S}} \times P_{T \Sigma} \times P_{\mathrm{S}} \times P_{\Sigma}, M_{c}\right) \cong \operatorname{Map}^{\mathbb{T}^{2}}\left(P_{T S} \times P_{\mathrm{S}}, \operatorname{Map}^{\mathbb{T}^{2}}\left(P_{T \Sigma} \times P_{\Sigma}, M_{c}\right)\right)
$$

Indeed, if $u \in \operatorname{Map}^{\mathbb{T}^{4}}\left(P_{T \mathrm{~S}} \times P_{T \Sigma} \times P_{\mathrm{S}} \times P_{\Sigma}, M_{c}\right)$ then the above isomorphism is the following map

$$
u \mapsto\left(p \mapsto u_{p}=u_{\left.\right|_{\{p\} \times P_{T \Sigma} \times P_{\Sigma}}}\right), \quad p \in P_{T \mathrm{~S}} \times P_{\mathrm{S}} .
$$

Now since we have a decomposition $\Omega^{0,1}(X)=\Omega^{0,1}(\Sigma) \oplus \Omega^{0,1}(S)$, it follows that both maps $u_{p}$ and $p \mapsto u_{p}$ are holomorphic: the map $u_{p} \in \operatorname{Map}^{\mathbb{T}^{2}}\left(P_{T \Sigma} \times\right.$ $\left.P_{\Sigma}, M_{c}\right)$ is considered as a holomorphic section of the corresponding bundle 
with respect to the connection $\hat{b}$ generated by the connection $b$ on $P_{\Sigma}$ and the Levi-Civita one on $P_{T \Sigma}$ and the map $p \mapsto u_{p}$ as a holomorphic map with respect to the connection $\hat{c}$ determined by the Levi-Civita connection of $P_{T \mathrm{~S}}$ and $c$. It means that the following system is satisfied:

$$
\begin{aligned}
& \bar{\partial}_{\hat{b}} u_{p}=0, \\
& \bar{\partial}_{\hat{c}} u=0 .
\end{aligned}
$$

Proposition 4.6.1. Suppose $(a, u) \in \mathcal{A}\left(P_{\text {det }}\right) \times \operatorname{Map}^{\mathbb{T}^{2}}\left(P_{r} \times P_{\text {det }}, M_{c}\right)$ is a solution of the generalized Seiberg-Witten equations over $X=\Sigma \times \mathrm{S}$ with its product metric. Then

$$
\left\{\begin{array}{l}
\bar{\partial}_{\hat{b}} u=0 \\
\bar{\partial}_{\hat{c}} u=0 \\
\left(d_{\mathrm{S}} b-d_{\Sigma} c\right) I_{S}-*_{\Sigma}\left(d_{\mathrm{S}} b-d_{\Sigma} c\right)=0 \\
*_{\Sigma} F_{b}+*_{S} F_{c}+\mu_{1} \circ u=0 .
\end{array}\right.
$$

Similarly as in the previous section one may consider metric (4.21) on $X$. In this case one obtains the following system

$$
\left\{\begin{array}{l}
\bar{\partial}_{\hat{b}} u=0 \\
\bar{\partial}_{\hat{c}} u=0 \\
\left(d_{\mathrm{S}} b-d_{\Sigma} c\right) I_{S}-*_{\Sigma}\left(d_{\mathrm{S}} b-d_{\Sigma} c\right)=0 \\
*_{\Sigma} F_{b}+\varepsilon *_{S} F_{c}+\mu_{1} \circ u=0 .
\end{array}\right.
$$

Hypothesis 4.6.2 ("Adiabatic limit conjecture"). There is a one-toone correspondence between solutions of the Seiberg-Witten equations 4.32) and solutions of the following system

$$
\left\{\begin{array}{l}
\bar{\partial}_{\hat{b}} u=0, \\
\bar{\partial}_{\hat{c}} u=0 \\
\left(d_{\mathrm{S}} b-d_{\Sigma} c\right) I_{S}-*_{\Sigma}\left(d_{\mathrm{S}} b-d_{\Sigma} c\right)=0, \\
*_{\Sigma} F_{b}+\mu_{1} \circ u=0 .
\end{array}\right.
$$

Further, consider the equations

$$
\left\{\begin{array}{l}
\bar{\partial}_{\hat{b}} \Psi=0, \\
*_{\Sigma} F_{b}+\mu_{1} \circ \Psi=0 .
\end{array}\right.
$$

for a pair $(b, \Psi) \in \mathcal{A}\left(P_{\Sigma}\right) \times \operatorname{Map}^{\mathbb{T}^{2}}\left(P_{T \Sigma} \times P_{\Sigma}\right)$. These are the vortex type equations over the Riemann surface $\Sigma$ as described in Section 4.4, Remark that 
the "additional twisting" in our case is the one determined by the principal bundle of $T \Sigma$ with its Levi-Civita connection. Let $\mathcal{M}_{T S V}^{0}$ denote the moduli space of 4.35), i.e. the space of solutions modulo $\mathcal{G}_{\Sigma}^{0}$.

One defines a principal $S^{1}$-bundle $P_{T S V} \rightarrow \mathcal{M}_{T S V}^{0}$ similarly to $P_{S V}$ of the previuos section. Namely, one considers first the following equations

$$
\left\{\begin{array}{l}
\bar{\partial}_{\hat{b}} u=0, \\
*_{\Sigma} F_{b}+\mu_{1} \circ \Psi-\frac{d}{\operatorname{Vol}(\Sigma)} \int_{\Sigma} \mu_{1} \circ \Psi d v o l_{\Sigma}=\frac{d}{\operatorname{Vol}(\Sigma)}+\tau,
\end{array}\right.
$$

where $\tau \in \mathbb{R}$ is a parameter. Denote by $\tilde{\mathcal{M}}_{T S V}$ the space of solutions modulo $\tilde{\mathcal{G}}_{\Sigma}$. The group $\mathcal{G}_{\Sigma}^{0} / \tilde{\mathcal{G}}_{\Sigma} \cong S^{1}$ acts on $\tilde{\mathcal{M}}_{T S V}$ with the momentum map $\tilde{\mu}$, namely

$$
\tilde{\mu}([b, \Psi])=\frac{1}{\operatorname{Vol}(\Sigma)} \int_{\Sigma} \mu_{1} \circ \Psi d_{v o l} .
$$

Therefore, we can define

$$
P_{T S V}=\tilde{\mu}^{-1}\left(-\frac{d}{\operatorname{Vol}(\Sigma)}-\tau\right) .
$$

The proof of the following theorem is analogous to the proof of Theorem 4.5.6 and uses Hypothesis 4.6.2.

Theorem 4.6.3. Let $X$ be a product of two holomorphic curves $\Sigma \times \mathrm{S}$ and $P_{\text {det }} \rightarrow X$ be given by (4.15). Suppose that Hypothesis 4.6.2 holds. Then there is one-to-one correspondence between equivariant holomorphig maps $f: P_{T S} \rightarrow \mathcal{M}_{T S V}^{0}$ such that $f^{*} P_{T S V} \cong P_{\mathrm{S}}$ and the moduli space

$$
\left.\mathcal{M}_{\text {SW }}^{0}=\left\{(a, u) \in \mathcal{A}_{h}\left(P_{\text {det }}\right) \times \operatorname{Map}^{H}\left(P_{\text {red }}, M_{c}\right) \mid(a, u) \text { solves } 4.7\right)\right\} / \mathcal{G}_{X}^{0},
$$

corresponding to the Seiberg-Witten system (4.7) over $\Sigma \times \mathrm{S}$.

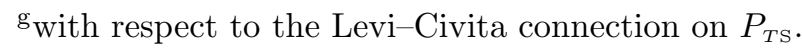




\section{Bibliography}

[1] D. V. Alekseevsky, S. Marchiafava, and M. Pontecorvo. Compatible almost complex structures on quaternion Kähler manifolds. Ann. Global Anal. Geom., 16(5):419-444, 1998.

[2] Damiano Anselmi and Pietro Fré. Topological $\sigma$-models in four dimensions and triholomorphic maps. Nuclear Phys. B, 416(1):255-300, 1994.

[3] Damiano Anselmi and Pietro Fré. Gauged hyperinstantons and monopole equations. Phys. Lett. B, 347(3-4):247-254, 1995.

[4] Michael Atiyah. New invariants of 3- and 4-dimensional manifolds. In The mathematical heritage of Hermann Weyl (Durham, NC, 1987), volume 48 of Proc. Sympos. Pure Math., pages 285-299. Amer. Math. Soc., Providence, RI, 1988.

[5] Michael Atiyah and Nigel Hitchin. The geometry and dynamics of magnetic monopoles. M. B. Porter Lectures. Princeton University Press, Princeton, NJ, 1988.

[6] Jonathan Bagger and Edward Witten. Matter couplings in $N=2$ supergravity. Nuclear Phys. B, 222(1):1-10, 1983.

[7] Fiammetta Battaglia. Circle actions and Morse theory on quaternionKähler manifolds. J. London Math. Soc. (2), 59(1):345-358, 1999.

[8] Marcel Berger. Sur les groupes d'holonomie homogenes de varietes a connexion affine et des varietes riemanniennes. Bull. Soc. Math. Fr., 83:279-330, 1955 .

[9] Roger Bielawski. Monopoles and the Gibbons-Manton metric. Comm. Math. Phys., 194(2):297-321, 1998.

[10] Roger Bielawski and Andrew S. Dancer. The geometry and topology of toric hyperkahler manifolds. Commun. Anal. Geom., 8(4):727-760, 2000. 
[11] Charles P. Boyer, Krzysztof Galicki, and Benjamin M. Mann. Quaternionic reduction and Einstein manifolds. Comm. Anal. Geom., 1(2):229279, 1993.

[12] Theodor Bröcker and Tammo tom Dieck. Representations of compact Lie groups, volume 98 of Graduate Texts in Mathematics. Springer-Verlag, New York, 1985.

[13] E. Calabi. Métriques kählériennes et fibrés holomorphes. Ann. Sci. École Norm. Sup. (4), 12(2):269-294, 1979.

[14] Jingyi Chen. Convergence of anti-self-dual connections on $\mathrm{SU}(n)$-bundles over product of two Riemann surfaces. Comm. Math. Phys., 196(3):571590, 1998.

[15] Jingyi Chen. Complex anti-self-dual connections on a product of Calabi-Yau surfaces and triholomorphic curves. Comm. Math. Phys., 201(1):217-247, 1999.

[16] Jingyi Chen and Jiayu Li. Quaternionic maps between hyperkähler manifolds. J. Differential Geom., 55(2):355-384, 2000.

[17] Kai Cieliebak, A. Rita Gaio, Ignasi Mundet i Riera, and Dietmar A. Salamon. The symplectic vortex equations and invariants of Hamiltonian group actions. J. Symplectic Geom., 1(3):543-645, 2002.

[18] Kai Cieliebak, Ana Rita Gaio, and Dietmar A. Salamon. J-holomorphic curves, moment maps, and invariants of Hamiltonian group actions. Internat. Math. Res. Notices, (16):831-882, 2000.

[19] S. K. Donaldson. Nahm's equations and the classification of monopoles. Comm. Math. Phys., 96(3):387-407, 1984.

[20] S. K. Donaldson and P. B. Kronheimer. The geometry of four-manifolds. Oxford Mathematical Monographs. The Clarendon Press Oxford University Press, New York, 1990. Oxford Science Publications.

[21] S.K. Donaldson. An application of gauge theory to four dimensional topology. J. Differ. Geom., 18:279-315, 1983.

[22] Stamatis Dostoglou and Dietmar A. Salamon. Self-dual instantons and holomorphic curves. Ann. of Math. (2), 139(3):581-640, 1994.

[23] Tohru Eguchi and Adrew J. Hanson. Asymptotically flat self-dual solutions to Euclidean gravity. Phys. Lett. B, 74:249-251, 1978. 
[24] Birte Feix. Hyperkahler metrics on cotangent bundles. J. Reine Angew. Math., 532:33-46, 2001.

[25] J. M. Figueroa-O'Farrill, C. Köhl, and B. Spence. Supersymmetric YangMills, octonionic instantons and triholomorphic curves. Nuclear Phys. B, 521(3):419-443, 1998.

[26] Ronald Fintushel and Ronald J. Stern. Knots, links, and 4-manifolds. Invent. Math., 134(2):363-400, 1998.

[27] Michael Hartley Freedman. The topology of four-dimensional manifolds. J. Differ. Geom., 17:357-453, 1982.

[28] Run Fueter. Die Funktionentheorie der Differentialgleichungen $\Delta u=$ 0 und $\Delta \Delta u=0$ mit vier reellen Variablen. Comment. Math. Helv., 7(1):307-330, 1934.

[29] K. Fukaya. Anti-self-dual equation on 4-manifolds with degenerate metric. Geom. Funct. Anal., 8(3):466-528, 1998.

[30] Rita Gaio, A. and Dietmar A. Salamon. Gromov-witten invariants of symplectic quotients and adiabatic limits. arXiv:math.SG/0106157, 2001.

[31] K. Galicki. A generalization of the momentum mapping construction for quaternionic Kähler manifolds. Comm. Math. Phys., 108(1):117-138, 1987.

[32] K. Galicki and H. B. Lawson, Jr. Quaternionic reduction and quaternionic orbifolds. Math. Ann., 282(1):1-21, 1988.

[33] Krzysztof Galicki and Yat Sun Poon. Duality and Yang-Mills fields on quaternionic Kähler manifolds. J. Math. Phys., 32(5):1263-1268, 1991.

[34] G. W. Gibbons and S. W. Hawking. Gravitational multi-instantons. Phys. Lett. B, 430, 1978.

[35] Werner Greub, Stephen Halperin, and Ray Vanstone. Connections, curvature, and cohomology. Vol. I: De Rham cohomology of manifolds and vector bundles. Pure and Applied Mathematics. Vol. 47. New York London: Academic Press. XIX, 443 p., 1972.

[36] N. J. Hitchin, A. Karlhede, U. Lindström, and M. Roček. Hyper-Kähler metrics and supersymmetry. Comm. Math. Phys., 108(4):535-589, 1987. 
[37] Nigel J. Hitchin. Metrics on moduli spaces. In Algebraic geometry, Proc. Lefschetz Centen. Conf., Mexico City/Mex. 1984, Pt. I, Contemp. Math. $58,157-178.1986$.

[38] Nigel J. Hitchin. The self-duality equations on a Riemann surface. Proc. Lond. Math. Soc., III. Ser., 55:59-126, 1987.

[39] Jacques Hurtubise. The classification of monopoles for the classical groups. Comm. Math. Phys., 120(4):613-641, 1989.

[40] Ignasi Mundet i Riera. Yang-mills-higgs theory for symplectic fibrations. arXiv:math.SG/9912150, 1999.

[41] Mitsuhiro Itoh. Quaternion structure on the moduli space of Yang-Mills connections. Math. Ann., 276(4):581-593, 1987.

[42] Dominic Joyce. Hypercomplex algebraic geometry. Quart. J. Math. Oxford Ser. (2), 49(194):129-162, 1998.

[43] D. Kaledin. Hyperkaehler structures on total spaces of holomorphic cotangent bundles. arXiv:alg-geom/9710026, 1997.

[44] A. G. Kovalev. Nahm's equations and complex adjoint orbits. Quart. J. Math. Oxford Ser. (2), 47(185):41-58, 1996.

[45] P. B. Kronheimer. A hyperkähler structure on the cotangent bundle of a complex lie group. arXiv:math.DG/0409253, 1988.

[46] P. B. Kronheimer. A hyper-Kählerian structure on coadjoint orbits of a semisimple complex group. J. London Math. Soc. (2), 42(2):193-208, 1990 .

[47] Peter B. Kronheimer and Hiraku Nakajima. Yang-Mills instantons on ALE gravitational instantons. Math. Ann., 288(2):263-307, 1990.

[48] H. Blaine Lawson, Jr. and Marie-Louise Michelsohn. Spin geometry, volume 38 of Princeton Mathematical Series. Princeton University Press, Princeton, NJ, 1989.

[49] Claude LeBrun. Einstein metrics and Mostow rigidity. Math. Res. Lett., 2(1):1-8, 1995.

[50] Claude LeBrun and Michael Singer. A Kummer-type construction of self-dual 4-manifolds. Math. Ann., 300(1):165-180, 1994. 
[51] Antony Maciocia. Metrics on the moduli spaces of instantons over Euclidean 4-space. Comm. Math. Phys., 135(3):467-482, 1991.

[52] Matilde Marcolli. Seiberg-Witten gauge theory, volume 17 of Texts and Readings in Mathematics. Hindustan Book Agency, New Delhi, 1999. With an appendix by the author and Erion J. Clark.

[53] Dusa McDuff and Dietmar Salamon. J-holomorphic curves and symplectic topology, volume 52 of American Mathematical Society Colloquium Publications. American Mathematical Society, Providence, RI, 2004.

[54] John D. Moore. Lectures on Seiberg-Witten invariants, volume 1629 of Lecture Notes in Mathematics. Springer-Verlag, Berlin, 1996.

[55] John W. Morgan. The Seiberg-Witten equations and applications to the topology of smooth four-manifolds, volume 44 of Mathematical Notes. Princeton University Press, Princeton, NJ, 1996.

[56] Liviu I. Nicolaescu. Notes on Seiberg-Witten theory, volume 28 of Graduate Studies in Mathematics. American Mathematical Society, Providence, RI, 2000.

[57] Takeo Nishinou. Convergence of Hermitian-Yang-Mills connections on Kähler surfaces and mirror symmetry. arXiv:math.SG/0301324, 2003.

[58] Ch. Okonek and A. Teleman. Gauge theoretical equivariant GromovWitten invariants and the full Seiberg-Witten invariants of ruled surfaces. Comm. Math. Phys., 227(3):551-585, 2002.

[59] V. Ya. Pidstrygach. Hyper-Kähler manifolds and the Seiberg-Witten equations. Tr. Mat. Inst. Steklova, 246(Algebr. Geom. Metody, Svyazi i Prilozh.):263-276, 2004.

[60] S. M. Salamon. Quaternion-Kähler geometry. In Surveys in differential geometry: essays on Einstein manifolds, Surv. Differ. Geom., VI, pages 83-121. Int. Press, Boston, MA, 1999.

[61] Simon Salamon. Riemannian geometry and holonomy groups, volume 201 of Pitman Research Notes in Mathematics Series. Longman Scientific \& Technical, Harlow, 1989.

[62] N. Seiberg and E. Witten. Electric-magnetic duality, monopole condensation, and confinement in $N=2$ supersymmetric Yang-Mills theory. Nuclear Phys. B, 426(1):19-52, 1994. 
[63] N. Seiberg and E. Witten. Monopoles, duality and chiral symmetry breaking in $N=2$ supersymmetric QCD. Nuclear Phys. B, 431(3):484550, 1994.

[64] A. Sudbery. Quaternionic analysis. Math. Proc. Camb. Philos. Soc., 85:199-225, 1979.

[65] Andrew Swann. HyperKahler and quaternionic Kahler geometry. Math. Ann., 289(3):421-450, 1991.

[66] Clifford Henry Taubes. The Seiberg-Witten invariants and symplectic forms. Math. Res. Lett., 1(6):809-822, 1994.

[67] Clifford Henry Taubes. More constraints on symplectic forms from Seiberg-Witten invariants. Math. Res. Lett., 2(1):9-13, 1995.

[68] Clifford Henry Taubes. The Seiberg-Witten and Gromov invariants. Math. Res. Lett., 2(2):221-238, 1995.

[69] Clifford Henry Taubes. Nonlinear generalizations of a 3-manifold's Dirac operator. In Trends in mathematical physics (Knoxville, TN, 1998), volume 13 of AMS/IP Stud. Adv. Math., pages 475-486. Amer. Math. Soc., Providence, RI, 1999.

[70] Robert Waldmüller. Gibbons-Hawking Räume und eine Verallgemeinerung der Seiberg-Witten Gleichungen. Diplomarbeit, 2003.

[71] Changyou Wang. Energy quantization for triholomorphic maps. Calc. Var. Partial Differential Equations, 18(2):145-158, 2003.

[72] Edward Witten. Monopoles and four-manifolds. Math. Res. Lett., 1(6):769-796, 1994.

[73] Joseph A. Wolf. Complex homogeneous contact manifolds and quaternionic symmetric spaces. J. Math. Mech., 14:1033-1047, 1965.

[74] Shing Tung Yau. Calabi's conjecture and some new results in algebraic geometry. Proc. Nat. Acad. Sci. U.S.A., 74(5):1798-1799, 1977.

[75] Shing Tung Yau. On the Ricci curvature of a compact Kähler manifold and the complex Monge-Ampère equation. I. Comm. Pure Appl. Math., 31(3):339-411, 1978. 


\section{Index}

$\bar{\partial}$-operator, 50

$\partial$-operator, 49

Adiabatic limit conjecture, 73,80

Aholomorphic

map, 53,55

section, 55

Aquaternionic

operator, 52

section, 55

Clifford multiplication, 16, 17,43

Determinant bundle, 44

Determinant line bundle, 16

Dirac operator, 7, 17

generalized, 45

Fundamental 4-form, 11

\section{Group}

$$
\begin{aligned}
& S_{0}^{1}, 20 \\
& S_{r}^{1}, 21 \\
& \operatorname{Sp}(1), 15 \\
& \operatorname{Spin}(4), 15 \\
& \operatorname{Spin}^{c}(4), 15 \\
& \text { gauge, } 7
\end{aligned}
$$

Harmonic spinor, 48

HyperKähler

reduction, 11

manifold, 10

potential, 20

Momentum map, 20
Permuting action, 19

Quaternionic Kähler manifold, 10

Quaternionic structure, 54

Representation

W, 16

Seiberg-Witten

moduli space, 7,18

equations, 7, 17

generalized equations, 60

invariant, 8

Standard $S p(1)$-representation, 42

Structure bundle, 38

Symplectic vortex equations, 65, 69

THC-system, 65 


\section{Lebenslauf}

\section{Andriy Haydys}

\section{Persönliche Daten}

geboren am 10.12.1979 in Lviv, Ukraine

Ukrainer, ledig

\section{Schulbildung}

1986-1994

1994-1997

\section{Studium}

1997-2002

Mathematikstudium an der Universität Lviv

Diplom Juni, 2002, mit Auszeichnung;

Note: ausgezeichnet

$7 / 2002-6 / 2005$

\section{Promotionsstipendium im Graduiertenkolleg der DFG}

Soroky-Lvivski Schule, Ukraine

Lyzeum der Physik und Mathematik bei Lviv, Ukraine; für besondere Erfolge im Studium mit Goldmedaille ausgezeichnet Gruppen und Geometrie an der Universität Göttingen

\section{Sonstige Tätigkeiten}

4/2004-9/2004 Wissenschaftliche Hilfskraft an der Universität Göttingen. Durchführung von Übungsgruppen zur Vorlesung Analysis II

seit 9/2005 Wissenschaftliche Hilfskraft an der Universität Göttingen. Durchführung von Übungsgruppen zur Vorlesung Einführung in der Topologie 